\title{
Spatiotemporal order out of noise
}

\author{
Francesc Sagués* \\ Departament de Química Física, Universitat de Barcelona, Diagonal 647, \\ 08028 Barcelona, Spain
}

\author{
José M. Sancho ${ }^{\dagger}$ \\ Departament d'Estructura i Constituents de la Matèria, Universitat de Barcelona, Diagonal \\ 647, 08028 Barcelona, Spain \\ Jordi García-Ojalvo ${ }^{\ddagger}$ \\ Departament de Física i Enginyeria Nuclear, Universitat Politècnica de Catalunya, Colom \\ 11, 08222 Terrassa, Spain
}

(Published 13 July 2007)

\begin{abstract}
Natural systems are undeniably subject to random fluctuations, arising from either environmental variability or thermal effects. The consideration of those fluctuations supposes to deal with noisy quantities whose variance might at times be a sizable fraction of their mean levels. It is known that, under these conditions, noisy fluctuations can interact with the system's nonlinearities to render counterintuitive behavior, in which an increase in the noise level produces a more regular behavior. In systems with spatial degrees of freedom, this regularity takes the form of spatiotemporal order. An overview is presented of the mechanisms through which noise induces, enhances, and sustains ordered behavior in passive and active nonlinear media, and different spatiotemporal phenomena are described resulting from these effects. The general theoretical framework used in the analysis of these effects is reviewed, encompassing the theory of stochastic partial differential equations and coupled sets of ordinary stochastic differential equations. Experimental observations of self-organized behavior arising out of noise are also described, and details on the numerical algorithms needed in the computer simulation of these problems are given.
\end{abstract}

DOI: 10.1103/RevModPhys.79.829

PACS number(s): 05.40.-a, 47.54.- r, 05.65.+b, 05.45.Xt

\section{CONTENTS}

I. Introduction: Order Out of Noise

A. Noise in macroscopic systems

1. Hydrodynamics patterns and turbulence

2. Liquid crystals

3. Chemical reactions

4. Electronic systems

5. Optical devices

6. Biophysical and biochemical systems

7. Other examples

B. Model scenarios

1. Discrete systems versus continuous media

2. Phase transitions

3. Pattern formation

4. Waves in active media

5. Synchronization: Local versus global coupling

C. Stochastic modeling

1. Stochastic differential equations

2. Internal versus external fluctuations

3. Additive versus multiplicative noise

*Electronic address: f.sagues@ub.edu

${ }^{\dagger}$ Electronic address: jmsancho@ecm.ub.es

†Electronic address: jordi.g.ojalvo@upc.edu
4. White versus colored noise

840

D. Constructive effects of noise in pure temporal dynamical systems

1. Noise-induced transitions

2. Stochastic resonance

3. Coherence resonance or stochastic coherence

4. Noise-induced transport

II. Methodological Approaches

A. Stochastic partial differential equations and lattice equations

1. Stochastic calculus: Stratonovich and Itô interpretations

2. The Fokker-Planck equation and statistical moments

B. Practical methodology

1. Mean-field approximation

2. Linear and nonlinear short-time analysis

3. Noise-effective models

4. Central-moment approximation

5. Numerical algorithms for white and structured noises

III. Nonequilibrium Phase Transitions

A. Continuous phase transitions

1. Short-time instabilities

2. Entropic mechanism and long-time analysis

3. The role of additive noise

4. Multiplicative-noise universality class 

B. First-order phase transitions
C. Dynamics of nonequilibrium phase transitions
1. Noise-induced fronts
2. Scaling regime
D. Spatiotemporally structured noises

IV. Stationary Patterns
A. Noisy precursors near pattern-forming bifurcations
B. Noise-induced patterns via a short-time instability
C. Entropy-driven patterns
D. Turing patterns

V. Spatiotemporal Dynamics: Noise-Unveiled Coherences A. Forced systems

1. Stochastic resonance in extended media

a. Discrete arrays

b. Continuous media

2. Noise-enhanced signal propagation

3. Spatiotemporal stochastic resonance

B. Autonomous systems

1. Stochastic coherence in extended media

2. Noise-triggered waves and noise-mediated front propagation

3. Spatial stochastic coherence

VI. Spatiotemporal Dynamics: Noise-Effective Regimes

A. Multiplicative noise effects in excitable systems

1. Excitability transitions: Experimental and numerical realizations

2. Excitability transitions: Nullcline-based scenarios

3. Other noise effects in active media

B. Doubly resonant effects

1. Doubly stochastic resonance

2. Signal propagation through monostable media

3. Stochastic coherence via symmetry restoring

VII. Synchronization and Control

A. Stochastic synchronization of active rotators

B. Stochastic synchronization of chaotic oscillators

C. Control of chaos by noise

1. Taming spatiotemporal chaos

2. Controlling scroll-wave chaos

VIII. Discussion

Acknowledgments

References
853

853

853

854

855

855

856

857

858

859

860

861

861

861

863

864

865

865

865

867

867

868

868

868

870

871

871

871

871

872

872

872

872

873

873

874

874

875

875

\section{INTRODUCTION: ORDER OUT OF NOISE}

Randomness is a common feature in our daily experience. Nature, either animate or inanimate, exhibits fluctuations at every scale we look at it. Whether we direct our efforts to scrutinize the natural world or we tailor more or less sophisticated experimental setups, the presence of uncertainties and noise is pervasive. Let us state from the beginning that noise, the central concept in this review, is going to be used as a generic term encompassing both thermally controlled internal fluctuations and parametric, or in general, environmental variability. Its origin, internal or external, will be distinguished in specific examples.

Classical statistical mechanics has developed canonical formalisms over the years, mostly intended to de- scribe the behavior of macroscopic systems in equilibrium, after averaging over a large number of, apparently uninteresting, random microscopic degrees of freedom. In systems operating far from equilibrium, on the other hand, random fluctuations have nontrivial effects, as shown by a large body of work that has been developed in the past three decades. The relevance of those results is to be better perceived, with the recent surge of interest in areas such as nanotechnology and cell biology. Those fields involve systems characterized by two features: they are small in size and exhibit a complex nonlinear behavior. The relative importance of fluctuations increases with decreasing system size, whereas the complex nonlinear dynamics interacts nontrivially with the noise. It is therefore necessary to bridge the gap between the advances in stochastic dynamics, mostly theoretical, that have been developed over recent years and newly developed experimental settings, such as those offered by the nanosciences and biosciences. This is the aim of the present review.

Whatever its origin, noise is usually considered, almost by definition, as a source of disorder in physical systems. However, it is well known that random fluctuations can conspire with nonlinearities to enhance regular behavior in simple time-dependent systems. There are two main ways in which this can happen. First, noise can help a multistable system to cross a potential barrier separating different stable states. If, for an optimal noise level, the (stochastic) crossing times statistically match a deterministic time scale (either internal or external) of the system, a more regular behavior may arise, in the form of a higher periodicity, for instance. This is the mechanism underlying phenomena such as stochastic resonance (Gammaitoni et al., 1998), coherence resonance (Lindner et al., 2004), and noise-induced transport (Reimann, 2002). Second, noise can destabilize existing steady states and induce new ones, which could correspond to states of higher regularity, via noise-induced transitions (Horsthemke and Lefever, 1984). In any of these two contexts, the system has to be nonlinear, or rendered effectively nonlinear by the noise terms.

In spatially extended systems, the local dynamics is coupled between neighboring sites, a feature that is conspicuously absent in purely temporal systems. Under these conditions, steady states become macroscopic phases, and transitions and bifurcations take the form of bona fide phase transitions. A description in terms of phases leads to the concept of order: certain phases can be interpreted as being more ordered than others, as a result of a coarse-grained description for which a zero field corresponds to a disordered mixture of different microscopic states, while a nonzero field arises as a coherent, ordered average of identical microscopic states. Within this framework, and as a generalization of the noise-induced transitions already mentioned, the past decade has witnessed the emergence of a wealth of examples in which noise induces phase transitions in spatially extended systems (García-Ojalvo and Sancho, 1999). More importantly and rather counterintuitively, 
an increase of noise intensity frequently leads to more ordered phases.

Order can also take the form of a spatially structured phase arising from a uniform, featureless one via a pattern-forming bifurcation. Evidence of such noisedriven patterns has been reported both theoretically and experimentally. More recently and again based on zerodimensional effects mentioned above, this time involving noise-mediated resonances, spatial coupling has also revealed itself to be an order-enhancing element in those situations in which regularity arises as a result of a resonant barrier crossing induced by noise.

This review provides a description of the ordering influence of noise in self-organizing extended systems. The present section is intended to highlight the most characteristic experimental setups in which the effects of spatiotemporal noise can be investigated. Although few experimental systems beyond prototypical ones mainly in chemical and electronic contexts have been extensively studied so far from this perspective, quite a number of ongoing realizations will be illustrated ranging from hydrodynamical to optical devices. In any case, special attention should be given to promising developments, especially in biophysical settings (see Sec. I.A.6), where the present level of control and modeling should foster the study of effects from either internal or external fluctuations. In the second part of Sec. I, typical theoretical models, basic stochastic concepts, and standard noiseinduced phenomenologies in nonextended dynamical systems are briefly reviewed in separate subsections. Section II presents an overview of the theoretical and numerical methodologies that have been developed in recent years to characterize noise-induced order. The scenarios leading to ordering noise-induced phase transitions and stationary patterns are described in Secs. III and IV, respectively. Section V reviews recent evidence of the constructive effect of spatial coupling in phenomena such as stochastic and coherence resonance, unveiling hidden coherences of the corresponding systems free from fluctuations. Section VI, on the other hand, discusses those cases, mostly in active media, in which noise-effective regimes enable us to interpret spatiotemporal dynamical behaviors arising purely out of noise. Finally, Sec. VII gives an overview of the active influence of noise on classical phenomena such as synchronization and control, and in particular discusses intriguing examples of chaos control by noise in different contexts.

\section{A. Noise in macroscopic systems}

A quantitative assessment of the ordering effects of noise in extended systems requires a careful control of the statistical properties of the fluctuations in time and, in some situations, also in space. To that end, different experimental settings have been designed in recent years that allow an adequate control of the driving forces acting on a variety of macroscopic systems. In some situations, the noise is internal and cannot be varied in a controlled way, but a joint experimental and theoretical analysis may allow a meaningful characterization of its

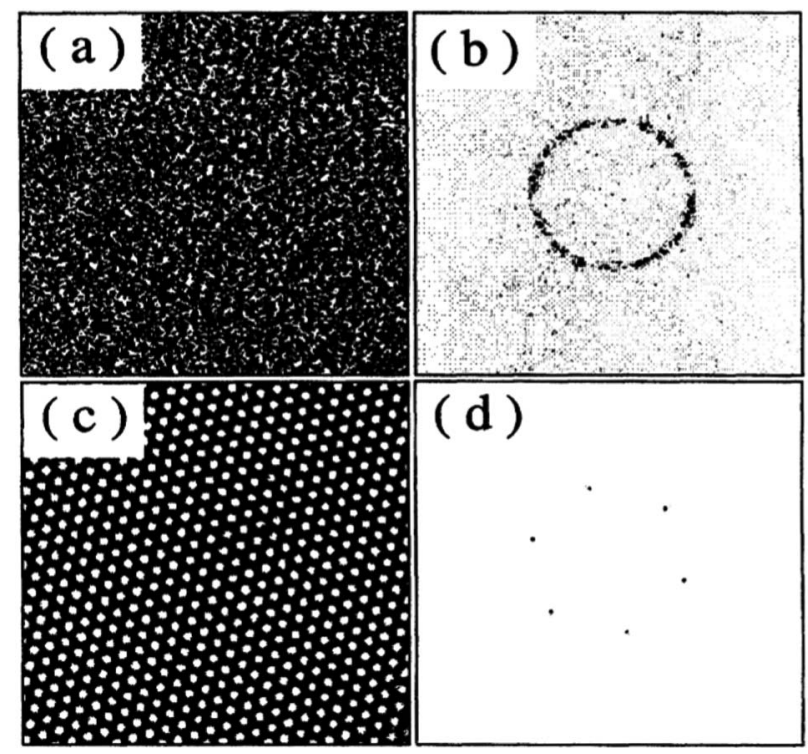

FIG. 1. Spatial noisy precursor in Rayleigh-Bénard convection. Experimental shadowgraph images (left) and the corresponding structure function (right) of a Rayleigh-Bénard cell below (top) and above (bottom) the onset of convection are shown. From Wu et al., 1995.

effect. In this section, we present an overview of different experimental implementations that have been used in these investigations, either because internal noise has macroscopic effects or because it is possible to introduce spatiotemporal external noise in a controlled way. The examples encompass different fields in physics, chemistry, and biology.

\section{Hydrodynamics patterns and turbulence}

Hydrodynamic systems are intrinsically spatially extended, and are naturally affected by random fluctuations, both thermal and external. Accordingly, they were among the first experimental systems in which the effects of spatiotemporal noise were studied. In RayleighBénard convection (Chandrasekhar, 1981), for instance, a transition separates different regimes of heat flow when a horizontal layer of fluid is heated from below. When fluctuations are ignored, the transition is sharp and continuous, with a diverging effective susceptibility similar to that found in second-order phase transitions. In order to determine whether these characteristics remained when the experimentally unavoidable thermal fluctuations were considered, Swift and Hohenberg (1977) developed a model (see Sec. I.B.3) that has since become canonical in nonequilibrium physics (Cross and Hohenberg, 1993).

Experimental studies of Rayleigh-Bénard convection allowed an indirect estimation of the amplitude of hydrodynamic fluctuations, by comparison with a stochastic Landau model (Meyer et al., 1987). A similar comparison was later performed with the stochastic SwiftHohenberg model (Hohenberg and Swift, 1992). Figure 1 shows experimental images of the vertical fluid flow below (a),(b) and above (c),(d) the convective instability 
threshold (Wu et al., 1995). The real field images show a disordered state below the bifurcation (a) and an ordered hexagonal phase in the convective regime (c). The structure function (defined as the Fourier transform of the correlation function) above threshold (d) exhibits a pattern of six well-defined maxima, hexagonally located. Below threshold (b), even though it corresponds to a disordered phase, the structure function displays an underlying preferred wavelength (with no preferred direction), anticipated by internal noise of hydrodynamic origin. These noisy precursors will be discussed in Sec. IV.A.

Besides pattern formation, hydrodynamic systems have also been used to study the effect of external fluctuations at the onset of turbulence. Gollub and Steinman (1980) applied a spatially uniform, temporally fluctuating temperature difference between the horizontal plates of a Rayleigh-Bénard cell as it was driven to turbulence.

Spatially uniform fluctuations have also been applied in experimental studies of a vertically vibrated horizontal fluid layer under the influence of parametric noise, and undergoing a Faraday instability (Berthet et al., 2003). In this case, as in the case of Rayleigh-Bénard convection described above, a controlled spatially uniform noise is relatively straightforward to apply as proven. However, the case of a spatiotemporal noise, fluctuating randomly also in space, is still challenging. A possible way of implementing such a noise source, in a somewhat related although different context, is by means of the thermal laser writing technique developed by Semwogerere and Schatz (2002) to imprint computercontrolled patterns on Bénard-Marangoni flows.

\section{Liquid crystals}

Nematic liquid crystals have long been used as model systems in pattern-forming experiments, due to their high controllability. This property also makes them useful for studying the effects of noise. Accordingly, early experiments were devoted to this issue, especially those regarding electroconvection. In this phenomenon, a convective flow of the liquid crystal sets in under the influence of an external electric field of sufficient intensity. Brand et al. (1985) studied the effect of a spatially uniform noise (superimposed to the external electric field) on the transition to turbulence in electroconvection. Their results showed, in contrast with those of Gollub and Steinman (1980) described previously, that noise substantially affected the threshold for the onset of turbulence, delaying it significantly. Rehberg et al. (1991) investigated the arousal of precursor patterns below, but close to, the onset of electroconvection, again driven by noise of internal origin. Shadowgraph images of the fluid flow in the liquid-crystal layer are shown in Fig. 2 both below and above the threshold of electroconvection. The noisy precursor that appears below threshold in the form of fuzzy rolls is clearly observed.

Electrohydrodynamic convection has also been used to study the induction of on-off intermittency by dichotomic noise (John et al., 1999). Once again, noise was
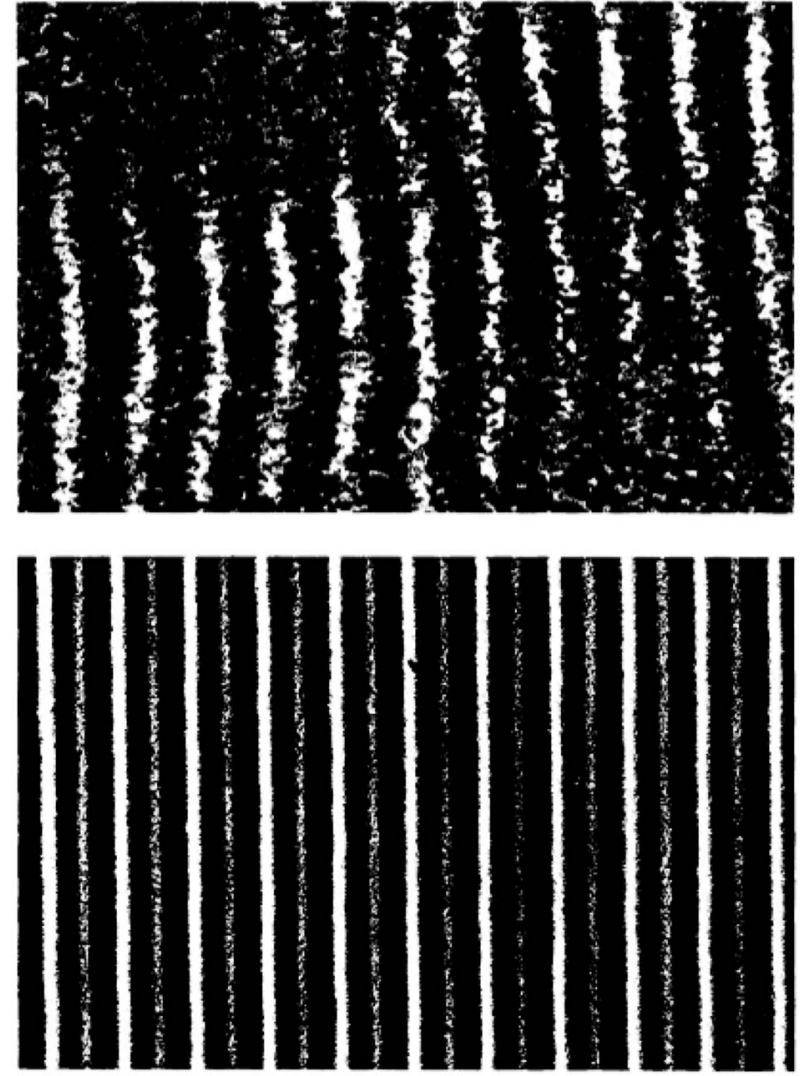

FIG. 2. Spatial structure of a nematic liquid crystal below (top) and above (bottom) the onset of electroconvection. From Rehberg et al., 1991.

applied uniformly in space. Finally, it should also be mentioned that, still for nematic materials, a simpler phenomenology has been studied that does not involve fluid flow instabilities. This is the so-called Freédericksz transition, in which only the orientation of the nematic director changes. The dynamics of fluctuations at onset was addressed by Sagués et al. (1988), whereas a complete phase diagram of this system, in the presence of a spatially uniform noise superimposed to an externally applied magnetic field, was determined by $\mathrm{Wu}$ and Andereck (1990).

\section{Chemical reactions}

As we have seen above, generating a controllable noisy forcing that fluctuates randomly not only in time, but also in space, is a challenging task. A much more advantageous perspective is offered by chemical systems, in particular by the photosensitive variant of the Belousov-Zhabotinsky (BZ) reaction (Kuhnert, 1986). In this system, the illumination level acts on the species ruthenium(II)-bipyridyl, a light-sensitive catalyst of the reaction, thereby controlling its excitability (Sagués and Epstein, 2003). By projecting a certain illumination profile, which can be easily generated by a computer, on a two-dimensional $\mathrm{BZ}$ reaction chamber, we gain a precise local control of the dynamical parameters of the system, with good resolution both in time and in space. In that 
way, noisy forcing with predetermined statistics and temporal and spatial correlation can be readily applied to the experimental system.

Early experimental observations of wave nucleation in media with disordered excitability, i.e., under timeindependent and space-dependent noise, were reported by Maselko and Showalter (1991) and Steinbock et al. (1995). These experiments, which were not designed specifically to address the effects of disorder on chemical waves, reported the presence of chemical BZ waves propagating in membranes in which the reaction catalyst had been imprinted with an ink jet printer. A bit later other studies followed, this time specifically devoted to analyzing the effects of external (parametric) noise on wave propagation. Sendiña-Nadal et al. (1998) investigated the effect of quenched disorder on the propagation of waves in a light-sensitive BZ medium. Figure 3 shows comparisons, for both one and two dimensions, of the propagation speed (upwards) of an initially planar wave, relating the cases of uniform (left side in each plot) and random (right) illumination profiles. Within the illumination range used, the speed of planar waves decreases linearly with the light intensity (SendiñaNadal et al., 1997). Static disorder was produced by spatial patches of random dichotomic illumination, with the same average light intensity as in the homogeneous part. Figure 3 shows that in the effectively one-dimensional setup (top plot), planar fronts moved slower under noisy illumination than in the homogeneous situation. Strikingly, the opposite behavior is found in the twodimensional configuration (bottom plot), with the front moving faster than without disorder.

Similar setups have been used profusely in recent years to study the effects of spatiotemporal noise in excitable media. Sendiña-Nadal et al. (2000), for instance, reported the Brownian motion of spiral waves induced by temporally correlated noise. Figure 4 shows results for three characteristic correlation times and fixed intensity of the noise. It is clear that the mobility of the spiral tip depends on the correlation time. For values of this correlation time different enough from $T / 2 \pi$, with $T$ the rotation period of the spiral wave, the trajectories have the form of entangled filaments and are confined to a bounded area of the medium. Contrarily, close to $T / 2 \pi$ the wandering motion spans over a larger region, eventually reaching the boundaries where the spiral wave finally dies out. The physical interpretation of this behavior is straightforward. At fixed noise intensity, for small correlation time the effect of random forcing must disappear, since fast, bounded fluctuations will be averaged out by the system. On the other hand, for large correlation times the spiral tip loses mobility, because the noise realization does not change appreciably during a rotation of the spiral wave. The effect of noise is thus remarkably enhanced at resonance with the rotation period.

Other examples where the photosensitive BZ reaction has been used are described in Secs. V and VI. Another light-sensitive chemical reaction that has been employed for this type of investigation is the Turing pattern-

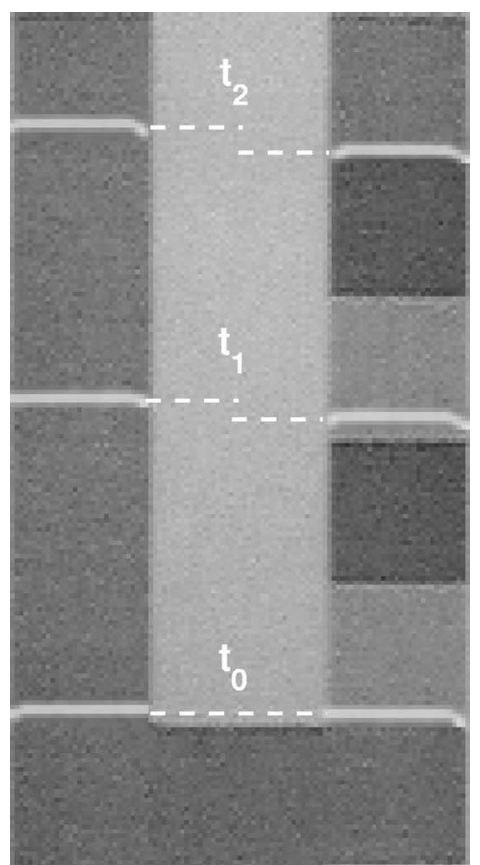

(a)

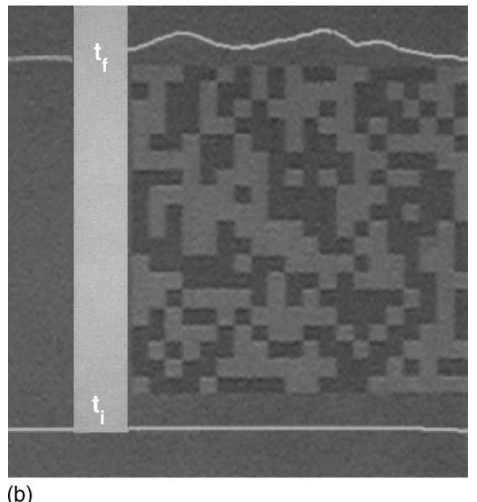

(b)

FIG. 3. Propagating BZ wave fronts on light-sensitive disordered media. (a) Quasi-one-dimensional setup and (b) twodimensional setup with randomly distributed square patches of illumination. The bright vertical stripe in the middle of each plot corresponds to an unexcitable region separating regions of uniform (left from the stripe) and random (right from the stripe) illumination. From Sendiña-Nadal et al., 1998.

forming CDIMA reaction (Muñuzuri et al., 1999). This reaction will be introduced in Sec. IV.

A different chemical context that has been analyzed in pattern-formation studies is that of heterogeneous catalysis and, in particular, the catalytic oxidation of $\mathrm{CO}$ on Pt single-crystal surfaces. This process has proven to be excitable under certain conditions, exhibiting a rich dynamical behavior (Imbihl and Ertl, 1995). Recently, local control of this process has been demonstrated using a mobile focused laser beam (Wolff et al., 2003). This type of result indicates that spatiotemporal random fluctuations can be induced in the temperature of the $\mathrm{Pt}$ surface in a controlled way. In a different development related with this experimental situation, Wehner et al. (2005) studied experimentally the role of different types 


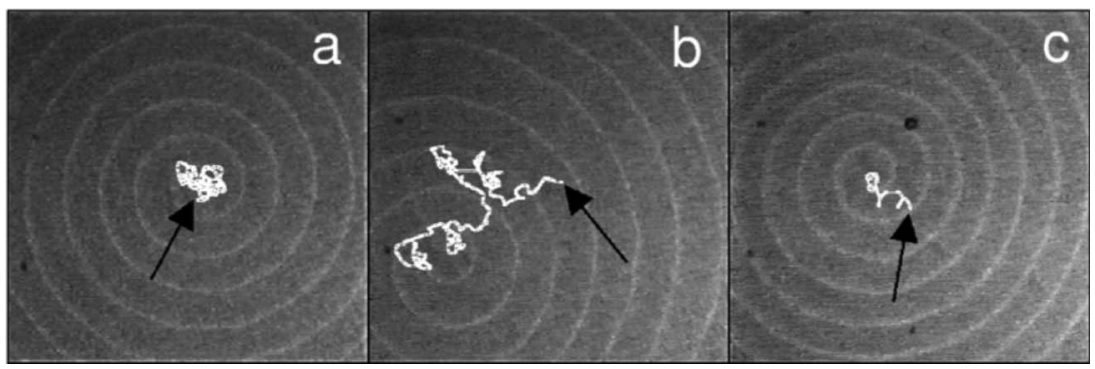

FIG. 4. Brownian motion of spiral waves in the noisy lightsensitive BZ reaction. Panels (a)-(c) display the whole tip trajectory for increasing values of the noise correlation time. The arrow indicates the initial position of the tip. From SendiñaNadal et al., 2000.

of noise on domain nucleation and growth in $\mathrm{CO}$ oxidation on Ir surfaces.

Finally and more oriented into a chemical engineering context, other instances of the interaction between patterning and noise are those related to turbulent mixing (Ottino, 1989; Reigada et al., 1997) and localized corrosion (Costa et al., 1991; Punckt et al., 2004).

\section{Electronic systems}

Besides being an interesting fundamental problem in itself, electronic noise in semiconductor structures has been recently harnessed to introduce random fluctuations in a controlled way in coupled arrays of electronic dynamical elements. Following a long tradition of using electronic circuits to study a wide variety of dynamical systems, ranging from classical chaotic oscillators (Carroll and Pecora, 1991) to neuronal dynamics (Nagumo et al., 1962), arrays of noise-driven electronic circuits have been used to demonstrate experimentally the existence of spatiotemporal stochastic resonance (Löcher et al., 1996), noise-enhanced propagation (Löcher et al., 1998), noise-enhanced synchronization (Lorenzo and Pérez-Muñuzuri, 1999), noise-induced excitability (Báscones et al., 2002), doubly stochastic resonance (Zaikin et al., 2001), and locking of coherence resonance oscillators (Han et al., 1999) among others.

The simplest way to generate well-controlled electronic noise is by amplifying the shot noise produced by a resistor or a diode. Such a noise generator is cost effective and compact enough to allow independent noise sources to be applied onto the different elements in an array of coupled electronic circuits. An example of an experimental setup using such a source of spatiotemporal noise is given in Fig. 5. In this case, Löcher et al. (1998) built a chain of 32 diode resonators coupled bidirectionally with their nearest neighbors by a simple resistance. Shot noise generated by $p n$ junction diodes was

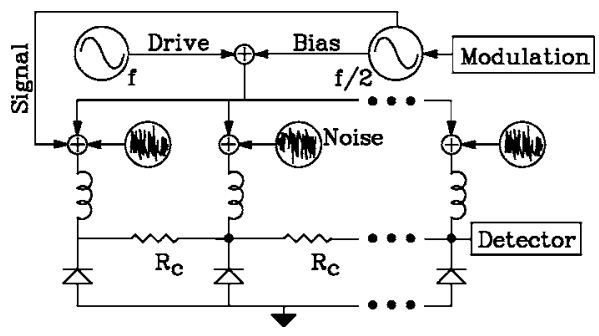

FIG. 5. Array of diode resonators driven by independent sources of electronic noise. From Löcher et al., 1998. applied to each one of the resonators, and noiseenhanced propagation was observed. For a higher control of the applied random fluctuations, computergenerated noise can be applied directly to the circuit, or commercially available function generators can be used. These solutions are, however, evidently less cost effective and flexible.

In a different type of study, investigations of noiseinduced phenomena in semiconductor nanostructures have been recently undertaken by means of theoretical models (Stegemann et al., 2005). It is to be expected that in the near future these studies will reveal novel constructive influences of noise in spatially extended nanostructures.

\section{Optical devices}

Nonlinear optical devices have been traditionally used as model systems in nonlinear dynamics, due to their high controllability in experiments and to the existence of theoretical models, stemming from first principles, that represent very accurately their behavior. The study of spatiotemporal dynamics requires broad-area optical setups, in which the transverse cross section of light beams (and the size of all associated optics) is sufficiently larger than any characteristic spatial scale. Broad-area optical systems have been used in recent years in pattern-formation studies, with potential applications in parallel information processing (Barland et al., 2002).

A particular system that has been used to study the effect of spatiotemporal noise in optical setups is based on the nonlinear optical properties of liquid crystals, specifically the dependence of their refraction index on the light intensity. This property allows the appearance of patterns in single feedback setups such as that shown in the top panel of Fig. 6. In that experiment (Louvergneaux et al., 2004), a broad beam of light propagates through a liquid-crystal layer, onto which it is injected back after being reflected off a mirror. It is well known that light propagation coupled with a light-intensitydependent refractive index leads to spatial patterns for large enough injected intensity, resulting from an absolute instability of the homogeneous state. In the presence of a small tilt of the mirror, a regime of convective instability sets in prior to the absolute instability. As predicted by Santagiustina et al. (1997), noise excites the system in the convectively unstable region, as shown in the bottom panel of Fig. 6. Similar results have been obtained in a free-electron laser, along the direction of 


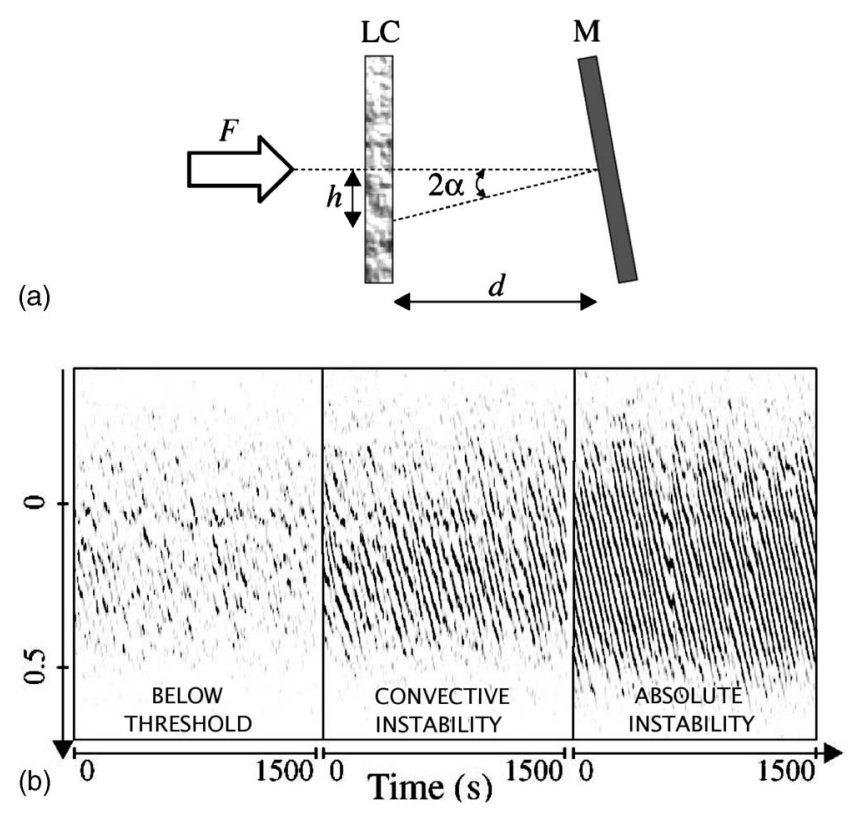

FIG. 6. Noise-driven convective instability in a liquid-crystal layer. Top: Single feedback mirror setup. Bottom: Spatiotemporal representation of the dynamics of a one-dimensional vertical section of the liquid-crystal layer. Space is represented vertically and time horizontally. Increasing intensities of the input laser beam are shown from left to right, leading to the regimes described in the plot. Adapted from Louvergneaux et al., 2004.

light propagation (Bielawski et al., 2005). In these cases, a comparison with theoretical predictions allows an estimate of the noise intensity, which, on the other hand, cannot be controlled externally in these setups.

Control of the applied spatiotemporal noise is possible, however, in a liquid-crystal setup, by acting optically upon the liquid-crystal layer. Sharpe et al. (2003) applied a random illumination pattern onto a liquidcrystal light valve by means of an additional liquidcrystal display, in order to investigate experimentally the occurrence of stochastic resonance in a two-dimensional system.

In a different context, it is worth mentioning the theoretical and experimental study of Vodonos et al. (2004). These authors proposed a thermodynamic interpretation of multiple pulse formation in passively mode-locked lasers, reporting (among other observations) the occurrence of a noise-induced phase transition in this system, with the noise coming from spontaneous-emission fluctuations that were injected from an external source.

\section{Biophysical and biochemical systems}

Biological systems at the cellular level are naturally subject to random fluctuations at different scales, ranging from the biochemical noise created by the inherent stochasticity of the reactions between biomolecules (which are usually present in the cell in small numbers) to the population noise created in neuronal tissue by thousands of background synapses impinging on a given neuron. As a result of this richness of phenomenological

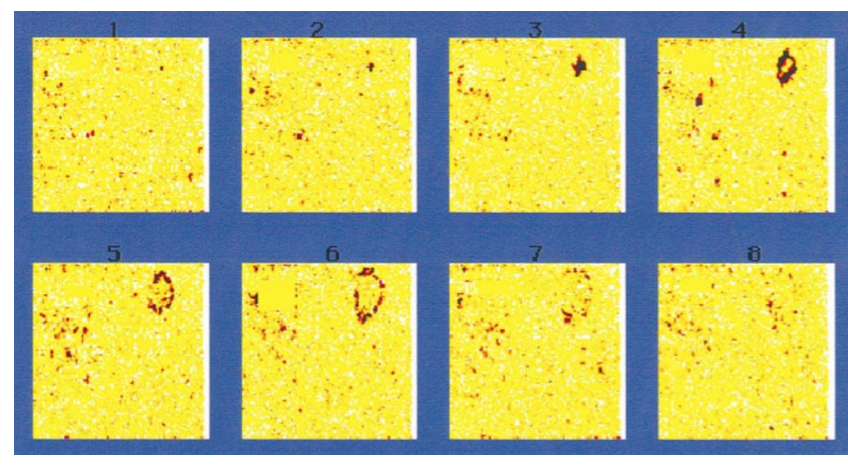

FIG. 7. (Color online) Snapshot sequences (time increasing from left to right and top to bottom) showing the concentration of calcium ions in a culture of glial cells. The authors interpret the structure at the top right corner at times 3-6 as a noise-induced wave. From Jung et al., 1998.

scenarios and motivations, a large number of studies have been devoted to determine the effect of noise on cellular behavior at many different levels. Particular attention must be paid to studies in stochastic resonance, at both the single-neuron (Gluckman et al., 1996) and behavioral (Russell et al., 1999) levels. Research into stochastic effects in gene expression has also acquired special relevance in recent years (Raser and O'Shea, 2005).

Less attention has been devoted, however, to spatiotemporal noise, at least from an experimental perspective, probably due to the difficulty, once more, of designing an experimental protocol capable of providing adequate control of externally applied fluctuations in this particular context. Jung et al. (1998) reported on the possibility of chemically controlling the level of spontaneous calcium activity, which they interpreted as background noise, in cultured glial cells. The control was obtained through the application of the neurotransmitter kainate at varying concentration levels: the general calcium activity increases with the kainate dose. Their results showed that under high activity conditions calcium waves were nucleated out of noise, as shown in Fig. 7.

The number of theoretical studies that have examined the role of spatiotemporal noise in the self-organization of cellular processes is larger. Pálsson and Cox (1996), for instance, used a stochastic model to describe the competition between circular and spiral waves observed experimentally in populations of Dictyostelium discoideum amoebae (Lee et al., 1996). Many theoretical efforts have also been devoted to study the role of stochasticity in intracellular calcium signaling (Shuai and Jung, 2002; Falcke, 2003). Discrete stochastic simulations have also been used to argue that biochemical noise could play a relevant role in the pole-to-pole oscillation of a certain protein that the bacterium Escherichia coli uses to determine its center (Howard and Rutenberg, 2003).

Recent biological setups offer the possibility of introducing experimentally spatiotemporal noise in a controlled way. We now describe two experimental systems in which the state of the art is ready for the introduction 

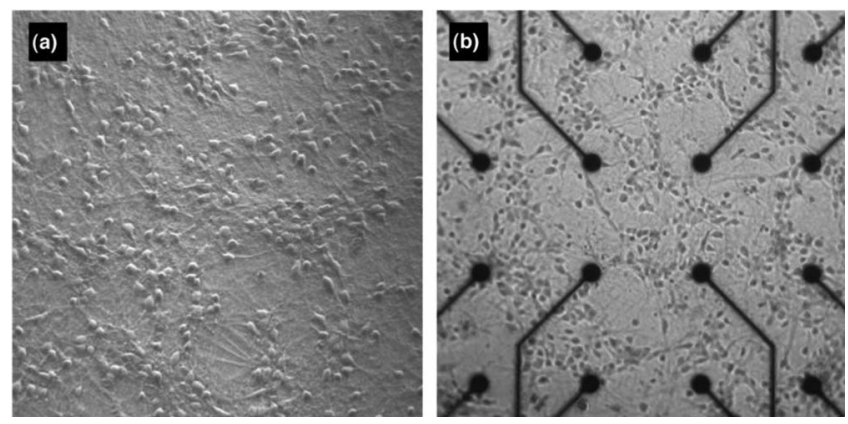

FIG. 8. Examples of two-dimensional neural cultures plated on (a) a glass coverslip and (b) a multielectrode array. Figure provided by E. Moses (Weizmann Institute of Science, Israel).

of noise. These systems fit very well with the methodology presented in this review.

In the first case (Limberis and Stewart, 2000), a large amount of kinesin molecular motors are deposited in a substrate that contains tubulin filaments, aligned in parallel and immobilized. When a microchip is laid on this construction, kinesins attach to it and initiate a direct transport or rotation of the microchip. Mathematically, this system consists of a set of independent degrees of freedom coupled globally through the microchip. This is an example of exact mean-field coupling. Fluctuations can be introduced through the control parameters of the system.

In the second example, a neural culture derived from dissociated rat hippocampus is prepared in a coverslip in one- or two-dimensional patterns (see Fig. 8). The connectivity between neurons is local. This system can present inhibitory, excitable, and oscillatory behavior, depending on the synaptic strength between neurons, which is chemically controlled. Signal propagation and information transport have been studied by Feinerman et al. (2005). Neural connectivity and the presence of a percolation transition in two-dimensional neural cultures have been examined by Breskin et al. (2006). These systems are sensitive to external inputs such as electromagnetic stimulation (Rotem and Moses, 2006), and are thus worthy candidates ready to be studied under the influence of controlled fluctuations.

Finally, novel visualization techniques are being developed for the monitoring of spatiotemporal activity in biological systems. These types of techniques should allow experimentalists to quantify the effect of fluctuations in extended biological systems. For example, propagation-induced phase contrast imaging allows the observation of excitable cardiac activity at high spatial resolution in a noninvasive way. Using that technique, Hwang et al. (2004) have observed contractile cellular motion, in the form of spiral waves, in cultured ventricle cells of rats (see Fig. 9). This type of spatiotemporal dynamics is analogous to that exhibited by the BelousovZhabotinsky reaction (see Sec. I.A.3), which as shown in this review has been substantially used in studies of noise-induced spatiotemporal order. Thus, one can expect that applying random perturbations in a controlled way to the system underlying Fig. 9 would lead to new

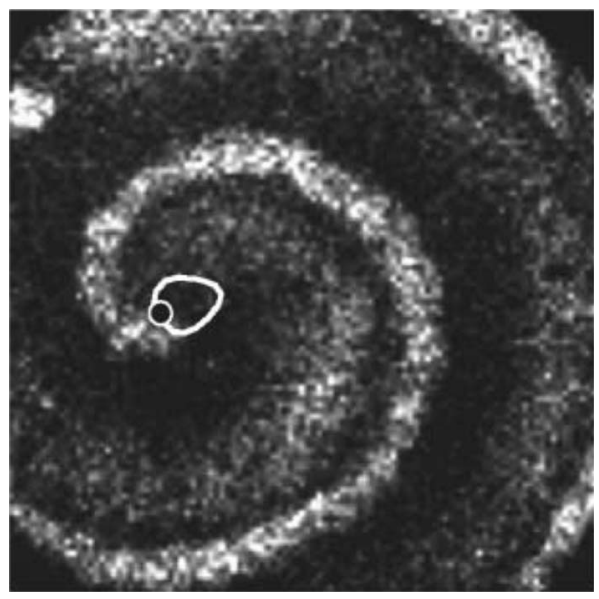

FIG. 9. Spiral wave of contractile activity in a culture of rat ventricle cells. From Hwang et al., 2004.

insights into the role of noise in biological media.

\section{Other examples}

Back to a physical context, two other situations in which the constructive effect of noise has been investigated in relation to pattern-forming systems are those of plasma physics (Dinklage et al., 1999) and interfacial solutal growth (González-Cinca et al., 2001).

On the other hand, the first theoretical example of a noise-induced phase transition was reported by Mikhailov (1979) on a population dynamics model of the Lotka-Volterra type, which has been frequently used in ecological modeling. A similar result was obtained more recently by Lai and Liu (2005).

Recently, studies of global-scale climate models have also addressed the influence of noise. Examples include the importance of stochastic resonance on millennialscale climate variability during glacial times (Ganopolski and Rahmstorf, 2002) and the occurrence of coherence resonance in those types of models (Perez-Muñuzuri et al., 2005).

\section{B. Model scenarios}

There is substantial evidence of self-organized behavior in spatially extended systems, as illustrated by previous examples. We review in this section a series of generic models that have been developed over the years to describe these phenomena.

\section{Discrete systems versus continuous media}

Spatially continuous extended media, such as fluids and chemical systems, are described by field variables obeying partial differential equations. A very common example is the reaction-diffusion equation, 


$$
\frac{\partial \phi(\mathbf{x}, t)}{\partial t}=f(\phi)+\mathcal{D} \nabla^{2} \phi
$$

where $\phi(\mathbf{x}, t)$ is a field (scalar or vector) that describes the state of the system at the spatial location $\mathbf{x}$ at time $t$. A discretization procedure is commonly used, as shown in Sec. II, to transform the continuous partial differential equation to be analyzed into a set of coupled ordinary differential equations, after approximating the continuous space by a lattice. In the case of Eq. (1), for example, assuming a regular Cartesian lattice, the discretization leads to

$$
\frac{d \phi_{i}(t)}{d t}=f\left(\left\{\phi_{i}\right\}\right)+\frac{\mathcal{D}}{\Delta x^{2}} \sum_{j \in \operatorname{nn}(i)}\left(\phi_{j}-\phi_{i}\right)
$$

where the sum term, which runs over the set of nearest neighbors of cell $i$, represents a possible choice for the discrete version of the Laplace operator and $\Delta x$ denotes the lattice spacing. ${ }^{1}$ The relation between the discretized field and the real one is $\phi_{i}(t)=\phi(i \Delta x, t)$, where $i$ $=\left(i_{1}, i_{2}, \ldots, i_{d}\right)$ and $d$ is the space dimension. The corresponding approach for stochastic differential equations is presented in Sec. II.A.

Formulations like that shown in Eq. (2) arise not only as approximations to continuous models, but also as basic descriptions of arrays of dynamical elements coupled locally to their neighbors. These types of models have been frequently considered in studies of noise-induced order in distributed media (see, for instance, Sec. V).

\section{Phase transitions}

Transitions between macroscopic phases provided the first examples of self-organized behavior in spatially extended systems (Stanley, 1971). Phase transitions take the system, upon variation of a given parameter, from a disordered state characterized by a zero average field to an ordered state with a nonzero average field or vice versa. The dynamics of phase transitions can be described in terms of models based on partial differential equations. The simplest one is the time-dependent Ginzburg-Landau model (Hohenberg and Halperin, 1977),

$$
\frac{\partial \phi}{\partial t}=a \phi-b \phi^{3}+\mathcal{D} \nabla^{2} \phi+\xi(\mathbf{x}, t)
$$

where $\xi(\mathbf{x}, t)$ stands for a spatiotemporal white noise representing thermal fluctuations. This model exhibits an equilibrium continuous transition from order to disorder when the temperature (noise intensity $\sigma^{2} \sim k_{B} T$ ), acting as the control parameter, is increased. Numerical simulations (Toral and Chakrabarti, 1990) located the critical point at $\sigma_{c}^{2} \approx 0.38$ for $a=b=1 / 2$ and $\mathcal{D}=2$, on a twodimensional square lattice. This phase transition is characterized by a behavior near the critical point that be-

\footnotetext{
${ }^{1}$ In what follows, we consider $\Delta x=1$ unless otherwise stated.
}

longs to the universality class of the two-dimensional Ising model. Other examples of phase transitions and their corresponding universality classes have been discussed in detail by Hohenberg and Halperin (1977). Here, in Sec. III, we focus mostly on nonequilibrium situations in which counterintuitively an increase of the external noise can induce a phase transition from a disordered phase to an ordered one.

\section{Pattern formation}

Perhaps the most clearly recognizable instance of spatial order is given by the emergence of stationary patterns from featureless states. The study of pattern formation out of equilibrium has been an active field of research in recent decades (Cross and Hohenberg, 1993). In its basic form, a stationary pattern arises out of a disordered state via an instability at zero frequency and nonzero wave number. A simple model exhibiting such bifurcation is the Swift-Hohenberg equation, first developed to describe the effect of hydrodynamic fluctuations near the onset of Rayleigh-Bénard convection (Swift and Hohenberg, 1977), and later extended to the deterministic case as a basic model of pattern formation (Cross and Hohenberg, 1993). The model reads

$$
\frac{\partial \phi}{\partial t}=a \phi-\phi^{3}-\left(\nabla^{2}+k_{0}^{2}\right)^{2} \phi+\xi(\mathbf{x}, t)
$$

where $\xi(\mathbf{x}, t)$ is an additive white noise as in Eq. (3) and $a$ denotes a control parameter. In the absence of noise, Eq. (4) has a trivial, homogeneous solution at $\phi=0$. The stability of this disordered phase can be studied by linearizing Eq. (4) and examining the temporal evolution of the Fourier mode $\phi(t)=\delta \phi \exp (i \mathbf{k} \cdot \mathbf{x}+\lambda t)$, where $\delta \phi \ll 1$. Substituting the latter expression into the linear version of Eq. (4) leads to $\lambda=a-\left(k_{0}^{2}-k^{2}\right)^{2}$. The trivial solution $\phi=0$ becomes unstable when $\lambda>0$; in particular, $k=k_{0}$ represents the most unstable wave number, and controls the wavelength of the emerging pattern at threshold $\left(a_{c}=0\right)$. Another length scale, corresponding to the average distance between defects as the pattern emerges, can be determined when the control parameter is slowly swept through the bifurcation point. This length happens to depend logarithmically on the additive noise intensity (Lythe, 1996).

An alternative phenomenology of pattern formation is associated to the well-known Turing instability (Turing, 1952), which applies to two-variable (activatorinhibitor) reaction-diffusion systems with long-range inhibition. The appropriate model we refer to here is the Epstein-Lengyel scheme adapted to the photosensitive version of the CDIMA (chlorine dioxide-iodinemalonic acid) reaction (Muñuzuri et al., 1999) mentioned in Sec. I.A.3,

$$
\frac{\partial u}{\partial t}=a-c u-4 \frac{u v}{1+u^{2}}-\phi(\mathbf{x})+\nabla^{2} u,
$$




$$
\frac{\partial v}{\partial t}=\sigma\left(c u-\frac{u v}{1+u^{2}}+\phi(\mathbf{x})+d \nabla^{2} v\right),
$$

where $u(\mathbf{x}, t)$ and $v(\mathbf{x}, t)$ represent the dimensionless concentrations of the species iodine and chlorine dioxide, respectively. The parameters $a, c, d$, and $\sigma$ are positive, and $\phi$ represents the illumination.

Noise effects in relation to both Swift-Hohenberg and Turing-like patterns will be addressed in Sec. IV.

\section{Waves in active media}

Self-organization can also take the form of spatiotemporal structures traveling in a coherent way through a spatially extended system. Such behavior is characteristic of excitable media, and gives rise to a large variety of propagating patterns, such as wavefronts, spiral waves, and scroll rings, depending on the dimensionality of the system (Mikhailov, 1994). A particular experimental realization of an excitable medium that will be discussed in this review is the Belousov-Zhabotinsky reaction, already mentioned in Sec. I.A.3, and in particular its photosensitive version, which allows for a direct application of controlled external spatiotemporal noise. This system can be described by the two-variable Oregonator model (Field et al., 1972; Tyson and Fife, 1980),

$$
\begin{aligned}
& \frac{\partial u}{\partial t}=\frac{1}{\epsilon}\left(u-u^{2}-[f v+\phi(\mathbf{x})] \frac{u-q}{u+q}\right)+\mathcal{D}_{u} \nabla^{2} u, \\
& \frac{\partial v}{\partial t}=u-v+\mathcal{D}_{v} \nabla^{2} v,
\end{aligned}
$$

where $u$ represents one of the intermediate species of the reaction and $v$ is the photosensitive ruthenium catalyst. $\mathcal{D}_{u}$ and $\mathcal{D}_{v}$ stand for the corresponding diffusion coefficients $\left(\mathcal{D}_{v}\right.$ is assumed zero hereafter to reproduce the fact that the catalyst is immobilized in the experimental setups that will be considered here). The parameters $f, q$, and $\epsilon$ are related to the kinetics of the $\mathrm{BZ}$ reaction. The multiplicative parameter $\phi$ accounts for the external forcing and is assumed directly proportional to the intensity of illumination.

A simplified version of the above-described model was developed by Barkley (1991) with the goal of obtaining a computationally efficient model for the numerical description of spatially extended excitable media. The Barkley model reads

$$
\begin{aligned}
& \frac{\partial u}{\partial t}=\frac{1}{\epsilon} u(1-u)\left(u-\frac{v+b}{a}\right)+\mathcal{D} \nabla^{2} u, \\
& \frac{\partial v}{\partial t}=u-v .
\end{aligned}
$$

In this model, the illumination enters via the parameter $b$. This system exhibits a transition between excitable and oscillatory local dynamics controlled by the parameter $b$.

Generically, activator-inhibitor systems are described by the pair of equations

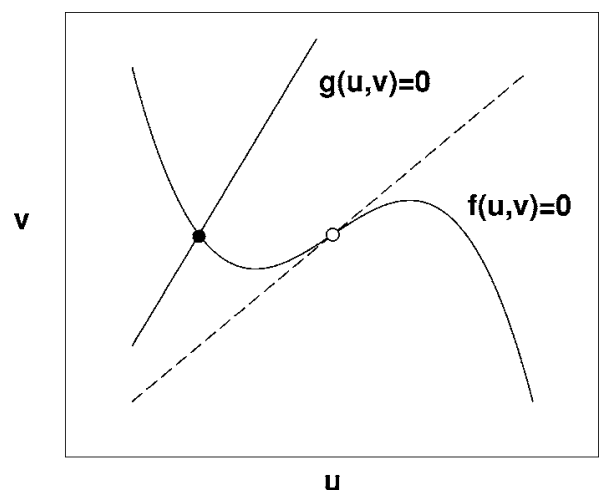

FIG. 10. Nullcline plots for a generic activator-inhibitor system. The cubic nullcline corresponds to $u$; the two straight lines are nullclines for $v$ in the cases of excitable (solid) and oscillatory (dashed) dynamics.

$$
\begin{aligned}
& \frac{\partial u}{\partial t}=\frac{1}{\epsilon} f(u, v)+\mathcal{D} \nabla^{2} u, \\
& \frac{\partial v}{\partial t}=g(u, v) .
\end{aligned}
$$

The corresponding nullcline plots for the zeros of the kinetic functions are shown in Fig. 10. Clearly their intersections locate the (single or multiple) steady states of the homogeneous system. Normally, the nullcline of the inhibitor variable is very simple, just a straight line, for instance. In contrast, the nullcline for the activator has a nonlinear shape that allows for multiple solutions. A single crossing at the left or right branches corresponds to excitable conditions, whereas a crossing at the middle (unstable) branch is a signature of oscillatory behavior. Triple crossings, as easily imagined, correspond to bistable situations. The effects of controlled external noise on different excitability regimes will be reviewed in Secs. V.B and VI.A.

\section{Synchronization: Local versus global coupling}

Another compelling example of self-organization in nonlinear systems is synchronization (Pikovsky et al., 2003). In particular, a great deal of attention has been paid to the synchronization of a large number of globally coupled oscillators. The standard model in this context is that of Kuramoto (1981),

$$
\frac{d \phi_{i}(t)}{d t}=\omega_{i}+\frac{C}{N} \sum_{j} \sin \left(\phi_{j}-\phi_{i}\right)
$$

This model describes the behavior of a set of $N$ coupled phase oscillators, whose phases are denoted by $\phi_{i}(t)$ and natural frequencies by $\omega_{i}$, assumed, for the sake of generality, to be distributed randomly. This model can be generalized, as described in Sec. VII.A, to represent the dynamics of excitable units (active rotators). Ignoring the effects of randomness on pure Kuramoto-like oscillators [for a recent reference, see Hong et al. (2005)], we limit this review and consider constructive effects of noise on arrays of active rotators under additive fluctua- 
tions. From another perspective, much effort has also been devoted to study the synchronization between noisy chaotic oscillators as well as to the synchronization of chaotic oscillators to an external (sometimes random) signal. Both global and local coupling realizations of these scenarios will be briefly reviewed in Sec. VII.B.

\section{Stochastic modeling}

Several issues must be taken into account when modeling random effects in spatially extended nonlinear systems. The generic framework to study these problems is the theory of stochastic processes. Moreover, since the phenomena of interest are normally time dependent, one has to think in terms of dynamical equations. Finally, given that the processes involved can be expected to have a very-well-controlled deterministic dynamical evolution, our stochastic tools have to be compatible with deterministic descriptions in a clear way. This reduces the number of possible theoretical descriptions: either Langevin-like stochastic differential equations for the relevant variables or dynamical equations for the probability density (master or Fokker-Planck equations) of these variables. Both approaches complement each other, and the choice of the more appropriate one depends on the specific observables.

In light of these considerations, our chosen approach will start by modeling a real system via deterministic differential equations and incorporate random forces in a subsequent, well-justified step. From this equation, one can derive the Fokker-Planck equation when necessary.

Within this generic framework, other points need some attention. Depending on the origin of the noise, one must distinguish between internal and external fluctuations, a distinction that is frequently closely related to that between additive and multiplicative noise. As will become evident throughout this review, these considerations are highly relevant for the influence that noise sources may have on a given system. Another important issue is the existence of correlations of the fluctuations in space and/or time. In some situations, the influence of these correlations is somewhat straightforward, while in other cases it is far from trivial.

\section{Stochastic differential equations}

Most of the systems we are interested in reside in a $d$-dimensional world. This means that our variables (fields or concentrations) depend on time and space. The starting deterministic models are based on partial differential equations, and when randomness is introduced we transform them into stochastic partial differential equations (SPDEs). A representative example is the deterministic reaction-diffusion equation,

$$
\frac{\partial \phi(\mathbf{x}, t)}{\partial t}=f(\phi(\mathbf{x}, t), \mu)+\mathcal{D} \nabla^{2} \phi(\mathbf{x}, t),
$$

where $\phi(\mathbf{x}, t)$ represents the density of a physical observable, $f(\phi(\mathbf{x}, t), \mu)$ is a nonlinear function of the field $\phi$, and $\mu$ denotes the relevant control parameter. The above example can be made more complicated when considering vector fields, higher-order derivatives, or nonlocal operators. The effect of fluctuations is introduced through a stochastic process or noise $\eta(\mathbf{x}, t)$ with well controlled statistical properties. As a result, we expect that the new equation governing our system will have the generic form

$$
\frac{\partial \phi}{\partial t}=f(\phi, \mu)+g(\phi) \eta(\mathbf{x}, t)+\mathcal{D} \nabla^{2} \phi .
$$

This is the most standard example of SPDE that will be analyzed here. ${ }^{2}$ Situations with noise entering nonlinearly are rather complicated to handle (Sagués et al., 1984; Santos et al., 2001) and are not going to be considered.

\section{Internal versus external fluctuations}

Randomness can have different origins in a real system. The unavoidable randomness associated with physical first principles gives rise to internal fluctuations. For very small systems, the quantum uncertainty principle fixes the order of magnitude of the smallest energy fluctuations $\hbar \omega$, where $\hbar$ is Planck's constant and $\omega$ is the characteristic frequency of the fluctuating variable or degree of freedom. A second source of randomness has a statistical origin and is related with the finite number of elements $N$ composing our system. These fluctuations are of order $N^{1 / 2}$. Finally, the second law of thermodynamics implies that any single element of a system in contact with a thermal bath at absolute temperature $T$ will exhibit energy fluctuations of magnitude $k_{B} T$, where $k_{B}$ is the Boltzmann constant.

Since we are dealing with macroscopic systems, we will not consider quantum fluctuations. Neither will we focus on the statistical fluctuations related with finite $N$, which are better studied using master equations. With respect to internal noise, our aim here is to consistently incorporate thermal fluctuations using the framework of SPDEs. The simplest and most well-founded method begins with the consideration of the deterministic relaxational model,

$$
\frac{\partial \phi}{\partial t}=-\Gamma \frac{\delta F}{\delta \phi},
$$

where $\Gamma$ stands for a kinetic coefficient representing dissipation and $F$ denotes a free energy or Lyapunov functional, which we consider to have the following form:

$$
F[\phi]=\int_{V}\left\{f(\phi)+\frac{1}{2}(\nabla \phi)^{2}\right\} d V .
$$

$f(\phi)$ denotes the local energy density and the squared gradient introduces a first-order coupling in space. The

\footnotetext{
${ }^{2}$ We use the notation of $\xi(\mathbf{x}, t)$ for additive and $\eta(\mathbf{x}, t)$ for multiplicative noises.
} 
equilibrium state of the system corresponds to the global minimum of this free-energy functional,

$$
\frac{\delta F}{\delta \phi}=0 .
$$

Thermal internal fluctuations are introduced by adding a noise term,

$$
\frac{\partial \phi}{\partial t}=-\Gamma \frac{\delta F}{\delta \phi}+\xi(\mathbf{x}, t)
$$

If the noise is Gaussian with zero mean and correlation,

$$
\left\langle\xi(\mathbf{x}, t) \xi\left(\mathbf{x}^{\prime}, t^{\prime}\right)\right\rangle=2 \Gamma k_{B} T \delta\left(\mathbf{x}-\mathbf{x}^{\prime}\right) \delta\left(t-t^{\prime}\right),
$$

one can prove that the probability distribution of the field $\phi$ obeys the expected Boltzmann equilibrium law,

$$
P([\phi]) \sim \exp \left\{-\frac{F[\phi]}{k_{B} T}\right\} .
$$

Actually the strategy of adding a noise term to a deterministic equation is of common use even in the absence of more fundamental statistical-mechanics-like arguments, because this is indeed the simplest way to explore the effects of fluctuations in a system where deterministic motion is well known or dominant.

A more interesting situation arises in experimental systems that have a well-established deterministic dynamics, but where some external parameter is allowed to fluctuate in a well-controlled way. Examples of these cases, some mentioned in Sec. I.A, are liquid crystals under fluctuating electromagnetic fields or photosensitive chemical reactions, including the BelouzovZhabotinsky and CDIMA reactions. In the general framework of Eq. (10), this situation is modeled by letting the control parameter $\mu$ fluctuate around a certain mean value,

$$
\mu \rightarrow \mu+\eta(\mathbf{x}, t)
$$

This leads to a SPDE of the generic form given by Eq. (11).

\section{Additive versus multiplicative noise}

From the previous analysis, one could conclude erroneously that internal noise is always additive and that external noise is multiplicative. This is frequently the case but with remarkable exceptions. In some particular situations, one can have multiplicative internal noise with a steady Boltzmann distribution (Ramírez-Piscina et al., 1993), or additive external noise, such as in the CDIMA reaction under the influence of a fluctuating illumination [see Eq. (5)] (Sanz-Anchelergues et al., 2001).

\section{White versus colored noise}

So far we have assumed, sometimes implicitly, that the noise terms were Gaussian and white. This is a very reasonable assumption for internal noise, which represents many irrelevant degrees of freedom evolving in very short temporal and spatial scales. Nevertheless, in real- istic experiments in which noise is introduced through some external device, one has to take into account the spatiotemporal structure of the fluctuations. One can then prescribe that noise is still Gaussian but with a correlation given, in its one-dimensional version, by

$$
\left\langle\eta(x, t) \eta\left(x^{\prime}, t^{\prime}\right)\right\rangle=\sigma_{0}^{2} C\left(\frac{\left|x-x^{\prime}\right|}{\lambda}, \frac{\left|t-t^{\prime}\right|}{\tau}\right),
$$

where $\sigma_{0}^{2}$ measures the intensity of the fluctuations and $C(x, t)$ is the correlation function, with $\lambda$ denoting the noise correlation length and $\tau$ its correlation time. A Gaussian white noise, such as that given by Eq. (16), is obtained in the limit $\lambda \rightarrow 0$ and $\tau \rightarrow 0$. This approximation simplifies the analytical calculations and is justified if the parameters $\lambda$ and $\tau$ are much smaller than any other temporal and spatial scales in the system. Two other interesting limits are worthy of comment. If we take the limit $\tau \rightarrow \infty$, we have a frozen noise $\eta(x)$, also known as spatial disorder. Instead, taking the limit $\lambda$ $\rightarrow \infty$, we account for global fluctuations $\eta(t)$. These last two cases are not easily handled when trying to get analytical results in $d$-dimensional problems and will be discussed only occasionally in this review.

\section{Constructive effects of noise in pure temporal dynamical systems}

Historically, the first scenarios in which the constructive role of fluctuations was reported were related to purely temporal dynamical systems modeled by the generic equation

$$
\frac{d q}{d t}=f(q)+g(q) \eta(t)
$$

where $q(t)$ denotes the relevant variable and $\eta(t)$ represents a Gaussian white noise, with temporal correlation,

$$
\left\langle\eta(t) \eta\left(t^{\prime}\right)\right\rangle=2 \sigma^{2} \delta\left(t-t^{\prime}\right) .
$$

The probability distribution $P(q, t)$ obeys a FokkerPlanck equation, written in the Stratonovich interpretation (see Sec. II.A.1) as

$$
\frac{\partial P}{\partial t}=-\frac{\partial}{\partial q} f(q) P+\sigma^{2} \frac{\partial}{\partial q} g(q) \frac{\partial}{\partial q} g(q) P,
$$

which has a steady distribution with natural boundaries,

$$
P_{\text {st }}(q)=\frac{N}{g(q)} \exp \left\{\int^{q} \frac{f\left(q^{\prime}\right)}{\sigma^{2} g^{2}\left(q^{\prime}\right)} d q^{\prime}\right\} \text {. }
$$

Using this simple formulation, it was found that noise can have ordering effects, which will now be recalled using several representative phenomena.

\section{Noise-induced transitions}

In the 1980s, noise was found to induce transitions in nonlinear systems by generating new maxima in the probability distribution function (Horsthemke and Lefe- 
ver, 1984). From the solution (23) one can obtain in a straightforward way the equation satisfied by the extrema of $P_{\mathrm{st}}$,

$$
f(q)-\sigma^{2} g^{\prime}(q) g(q)=0 .
$$

Equation (24) shows that in the presence of multiplicative noise [i.e., provided that $g(q)$ is not constant] new extrema may arise that are different from the deterministic solutions $f(q)=0$. The appearance of new extrema, and/or the disappearance of old ones, at some critical value of $\sigma^{2}$ can be interpreted as a noise-induced transition. In spatially extended systems, noise-induced transitions between macroscopic phases can arise. This phenomenology will be reviewed in Sec. III.

\section{Stochastic resonance}

In a second breakthrough, random forcing was seen to enhance the response of bistable systems to weak periodic driving, a popular phenomenon that came to be known as stochastic resonance (SR) (Wiesenfeld and Moss, 1995). The situation was later extended to other types of driving signals (not only periodic) and nonlinear systems (not only bistable), as discussed by Gammaitoni et al. (1998) and Anishchenko et al. (1999). The simplest model for SR is

$$
\frac{d q}{d t}=q-q^{3}+A_{0} \cos (\Omega t+\theta)+\eta(t),
$$

which exhibits bistability between the states $q_{m}= \pm 1$, in the absence of external signal $\left(A_{0}=0\right)$. One can check numerically, and prove analytically, that the system is entrained to the periodic perturbation even for small amplitudes, provided the intensity of the noise is large enough. In this sense, noise is able to amplify the small amplitude $A_{0}$ of the forcing to produce a large periodic response of amplitude $q_{m} \gg A_{0}$. Different quantities have been proposed to quantify this phenomenon, the most extended of which relies on the calculation of the signal-to-noise ratio at the input frequency from the power spectral density of the output signal. This observable exhibits a maximum for intermediate values of the noise intensity, indicating an optimal response induced by noise. The influence of spatial coupling on this behavior will be reviewed in Secs. V and VI.

\section{Coherence resonance or stochastic coherence}

An external driving signal is in fact not necessary to organize a temporally coherent output in a stochastic nonlinear system, provided the system has itself an intrinsic time scale. This is the case of noisy precursors near nonlinear instabilities of periodic orbits (Wiesenfeld, 1985; Neiman et al., 1997). Probably the simplest example is that provided by the dynamics of a single variable in a tilted periodic potential (Sigeti and Horsthemke, 1989). A two-variable systems-based description of this phenomenon was proposed quite a long time ago (Gang et al., 1993) and particularly in relation to activator-inhibitor models operating in excitable re- gimes but close to a Hopf bifurcation (Pikovsky and Kurths, 1997). In this case, the system exhibits for some intermediate values of the noise intensity a quasioscillatory behavior, i.e., there is a noticeable degree of coherence of the output signal without any input signal. If no noise is present, the system displays a constant steady state. This noise-induced coherence has been called autonomous or internal stochastic resonance, or, popularly, coherence resonance, although we believe that the term stochastic coherence is more appropriate and use it throughout this review. The standard scheme is the FitzHugh-Nagumo model, which takes the form (Pikovsky and Kurths, 1997)

$$
\begin{aligned}
& \frac{d x}{d t}=x-\frac{x^{3}}{3}-y, \\
& \varepsilon \frac{d y}{d t}=x+a+\xi(t) .
\end{aligned}
$$

For small values of $\varepsilon$ and $a$ slightly larger than unity, the variable $x$ exhibits spikes at certain random times whose statistical characteristics depend on the noise intensity, exhibiting stochastic coherence.

A measure of the degree of coherence between the pulses is the coherence parameter $R$,

$$
R=\frac{\sqrt{\left\langle\Delta t_{i}^{2}\right\rangle}}{\left\langle t_{i}\right\rangle}
$$

where $t_{i}$ denotes the interspike interval preceding pulse $i$ and $\left\langle\Delta t_{i}^{2}\right\rangle$ stands for the variance of this random process. This parameter gives an idea of the coherence or periodicity of the pulses. What one observes is that for a finite value of the noise intensity this parameter has a minimum, thus indicating an optimal degree of coherence. The result is that noise unveils a characteristic time scale hidden in the system. Similar phenomena in spatially extended systems will be described in Sec. V.

\section{Noise-induced transport}

A final phenomenon of noise-induced regularity in pure dynamical systems is the appearance of transport phenomena due to the presence of fluctuations in systems far from equilibrium. Here we simply mention two situations without further discussion. The first case involves the overdamped motion of a particle on a periodic asymmetric (ratchet) and flashing potential under the action of an additive white noise. In the stationary regime, the particle exhibits a nonzero average velocity, a counterintuitive result given the lack of an external driving force. Several versions of this so-called ratchet effect exist, involving different conditions for the potential and the noise that lead to both spatial and temporal symmetry breaking.

The second case is represented by particles in the above-mentioned tilted potentials. In the absence of noise, particles will remain forever trapped in a local minimum, but when the additive noise is present they can escape, overcoming the barrier and creating in this 
way a net material flux. A thorough description of both phenomena can be found in the review by Reimann (2002).

\section{METHODOLOGICAL APPROACHES}

We introduce in this section Langevin and FokkerPlanck equations for systems with spatial degrees of freedom, and discuss analytical and numerical approaches that allow us to extract information from the highly intractable equations that describe stochastic nonlinear media.

\section{A. Stochastic partial differential equations and lattice equations}

Stochastic differential, ordinary or partial, equations can be derived in several ways, ranging from ad hoc proposals to more elaborate methods using, for instance, master equation expansions. A generic model, representing most of the situations described in the Introduction, can be written, in a slightly more compact form than in Eq. (11), as

$$
\frac{\partial \phi}{\partial t}=f(\phi, \nabla)+g(\phi, \nabla) \eta(x, t),
$$

where $\phi(x, t)$ is a field or density variable at point $x$ and time $t$. The deterministic term $f(\phi, \nabla)$ is a function of the field and its spatial derivatives $\nabla=\partial / \partial x$, and $\eta(x, t)$ denotes a spatiotemporal random process or noise whose statistical properties need to be specified. Usually this noise can be assumed to be Gaussianly distributed and, without loss of generality, to have zero mean. Its spatiotemporal correlation function has the generic form given by Eq. (19).

A Gaussian white noise is obtained in the limit $\lambda \rightarrow 0$ and $\tau \rightarrow 0$, for which the correlation (19) reduces to

$$
\left\langle\eta(x, t) \eta\left(x^{\prime}, t^{\prime}\right)\right\rangle=2 \sigma^{2} \delta\left(x-x^{\prime}\right) \delta\left(t-t^{\prime}\right) .
$$

The function $g(\phi, \nabla)$ represents the coupling with the noise, which can be explicitly dependent on the field (multiplicative noise) or not (additive noise), and can also include spatial derivatives.

For practical simulation purposes and mathematical simplification, one can work in a lattice and take the continuous limit when necessary. Dividing space into $N$ cells of size $\Delta x$, Eq. (28) can be rewritten as

$$
\frac{d \phi_{i}(t)}{d t}=f_{i}(\{\phi\})+g_{i j}(\{\phi\}) \eta_{j}(t),
$$

where indices $i$ and $j=1, \ldots, N$ denote the cell location, ${ }^{3}$ so that $\phi_{i}(t)=\phi(i \Delta x, t)$, and $\{\phi\}$ represents the set of $\phi_{i}$ variables. Discretization of the noise correlation (19) is immediate, and in the white noise case Eq. (29) leads to

\footnotetext{
${ }^{3}$ Throughout it is assumed that repeated indices are summed from 1 to $N$, except where indicated. In some cases, summations will be written explicitly for the sake of emphasis.
}

$$
\left\langle\eta_{i}(t) \eta_{j}\left(t^{\prime}\right)\right\rangle=2 \frac{\sigma^{2}}{\Delta x} \delta_{i j} \delta\left(t-t^{\prime}\right) .
$$

These transformations reduce the SPDE (28) to a set of coupled SODE's (30).

The description given above has been restricted for simplicity to a one-dimensional space, but extension to a $d$-dimensional space is straightforward. Examples of the transformations between continuous and discrete descriptions can found exhaustively throughout this review.

\section{Stochastic calculus: Stratonovich and Itô interpretations}

Studies on SDEs have established the need of carefully interpreting stochastic integrals involving multiplicative noise terms white in time (Stratonovich, 1967; Horsthemke and Lefever, 1984; Gardiner, 1989). This problem appears in different forms; here we discuss it in relation with the derivation of algorithms for numerical integration. This approach will also be very useful in the derivation of Fokker-Planck equations.

We begin by performing a formal integration of Eq. (30) for a generic kind of noise,

$$
\begin{aligned}
\phi_{i}(t+\Delta t)= & \phi_{i}(t)+\int_{t}^{t+\Delta t} f_{i}\left(\left\{\phi\left(t^{\prime}\right)\right\}\right) d t^{\prime} \\
& +\int_{t}^{t+\Delta t} g_{i j}\left(\left\{\phi\left(t^{\prime}\right)\right\}\right) \eta_{j}\left(t^{\prime}\right) d t^{\prime} .
\end{aligned}
$$

A naive expansion at $t^{\prime}=t$ of the functions $f_{i}$ and $g_{i j}$ would lead to

$$
\begin{aligned}
\phi_{i}(t+\Delta t)= & \phi_{i}(t)+f_{i}(\{\phi(t)\}) \Delta t+g_{i j}(\{\phi(t)\}) \mathcal{X}_{j}(t) \\
& +\cdots,
\end{aligned}
$$

where $\mathcal{X}_{i}(t)$ is a new stochastic process, defined by the integral

$$
\mathcal{X}_{j}(t) \equiv \int_{t}^{t+\Delta t} \eta_{j}\left(t^{\prime}\right) d t^{\prime} .
$$

This simple algorithm is correct up to first order in $\Delta t$, provided the noise has temporal structure (colored noise), but it is incorrect if the noise is delta correlated (white noise) as in Eq. (31), which is the most common assumption.

The white-noise hypothesis is founded on a sound physical argument for noise representing unknown degrees of freedom, which usually evolve in temporal and spatial scales much shorter than those of the more relevant variables of the system. Moreover, the assumption of a delta correlation in time implies that the corresponding stochastic process is Markovian, which is indeed an important simplification from the point of view of the theory of stochastic processes. The price to pay is that the stochastic integral appearing in the last term of Eq. (32) is not uniquely defined. As a consequence, the algorithm defined by Eq. (33) has to be revised. As a matter of fact, when the noise is white in time, the stochastic integral defined in Eq. (34) is the definition of the 
Wiener increment $\mathcal{X}_{i}(t)=\Delta W_{i}(t)$, which is the simplest known Gaussian process. It has zero mean and its second moment is

$$
\left\langle\Delta W_{i}(t) \Delta W_{j}(t)\right\rangle=2 \delta_{i j} \frac{\sigma^{2}}{\Delta x} \Delta t
$$

The first immediate conclusion is that the integral (34) is of order $\Delta t^{1 / 2}$, and then the algorithm (33) has to be completed, to account for further contributions of order $\Delta t$ coming from the noise term.

Actually, other contributions of the noise term depend on the interpretation of the stochastic integral in Eq. (32), which can be defined using the Riemann formula,

$$
\begin{aligned}
\int_{t}^{t+\Delta t} g_{i j}\left(\left\{\phi\left(t^{\prime}\right)\right\}\right) \eta_{j}\left(t^{\prime}\right) d t^{\prime}= & g_{i j}(\{(1-\alpha) \phi(t) \\
& +\alpha \phi(t+\Delta t)\}) \Delta W_{j}(t),
\end{aligned}
$$

with $0 \leq \alpha \leq 1$ (Gardiner, 1989; van Kampen, 1992). Since the order in $\Delta t$ of this integral is $\alpha$ dependent, one needs to make a choice of $\alpha$, known as a stochastic interpretation.

Using Eq. (33), we can estimate that to lowest order in $\Delta t^{1 / 2}$,

$$
\phi_{k}(t+\Delta t)=\phi_{k}(t)+g_{k l} \Delta W_{l}(t)+\cdots,
$$

and from this result we find the two lowest contributions to Eq. (36),

$$
\begin{aligned}
\int_{t}^{t+\Delta t} & g_{i j}\left(\left\{\phi\left(t^{\prime}\right)\right\}\right) \eta_{j}\left(t^{\prime}\right) d t^{\prime} \\
\quad= & g_{i j}(\{\phi(t)\}) \Delta W_{j}(t)+\alpha \frac{\partial g_{i j}}{\partial \phi_{k}} g_{k l} \Delta W_{l}(t) \Delta W_{j}(t)+\cdots .
\end{aligned}
$$

Substituting this expression into Eq. (32) leads to the correct version of algorithm (33) up to first order in $\Delta t$,

$$
\begin{aligned}
\phi_{i}(t+\Delta t)= & \phi_{i}(t)+f_{i}(\{\phi(t)\}) \Delta t \\
& +g_{i j}(\{\phi(t)\}) \mathcal{X}_{j}(t)+\alpha \frac{\partial g_{i j}}{\partial \phi_{k}} g_{k l} \mathcal{X}_{l}(t) \mathcal{X}_{j}(t),
\end{aligned}
$$

which does depend on $\alpha$ and is valid for any stochastic interpretation. Higher-order algorithms for white noise and structured noises are presented at the end of this section.

The most common interpretations are $\alpha=0$ (Itô interpretation) and $\alpha=1 / 2$ (Stratonovich interpretation). The first one is commonly used in the mathematical literature, and is simpler in the sense that the value of the stochastic integral (36) depends only on the value of the field at the lower limit of the integration interval. A rigorous set of results exist under this interpretation, but the standard rules of calculus cannot be used in this case. The Stratonovich interpretation, on the other hand, is more common in the physical literature, because it has the correct behavior in the limit of vanishing correlation time $\tau \rightarrow 0$. This is usually the case of experimental noises, which are naturally correlated in time but whose correlation time might be very small. Furthermore, the standard rules of calculus for derivatives and integrals can be used within this interpretation.

\section{The Fokker-Planck equation and statistical moments}

We consider here a noise white in time but structured (i.e., correlated) in space. In a discrete space, the noise correlation (19) can be written as

$$
\left\langle\eta_{i}(t) \eta_{j}\left(t^{\prime}\right)\right\rangle=c_{i j} 2 \delta\left(t-t^{\prime}\right) .
$$

The Fokker-Planck equation obeyed by the probability density $P(\{\phi\}, t)$ can be obtained using the KramersMoyal expansion of the Chapman-Kolmogorov equation (Stratonovich, 1967; Risken, 1984; Gardiner, 1989; García-Ojalvo and Sancho, 1999),

$$
\begin{aligned}
\frac{\partial P(\{\phi\}, t)}{\partial t}= & -\frac{\partial}{\partial \phi_{i}} K_{i}(\{\phi\}) P+\frac{1}{2} \frac{\partial^{2}}{\partial \phi_{i} \partial \phi_{l}} K_{i l}(\{\phi\}) P \\
& +\cdots,
\end{aligned}
$$

where $K_{i}$ and $K_{i j}$ are the first two differential moments. The first term on the right-hand side of Eq. (41) is called the drift term, the second one is referred to as the diffusion term, and the unwritten contributions correspond to higher-order moments. We can take advantage of the algorithm defined before, using Eqs. (33), (39), and (40), to evaluate the first two moments. Defining the increment $\Delta \phi_{i}=\phi_{i}(t+\Delta t)-\phi_{i}(t)$, the first moment reads

$$
\begin{aligned}
K_{i}(\{\phi\}) & =\left.\lim _{\Delta t \rightarrow 0} \frac{\left\langle\Delta \phi_{i}\right\rangle}{\Delta t}\right|_{\phi(t) \rightarrow \phi} \\
& =f_{i}(\{\phi\})+2 \alpha \frac{\partial g_{i j}}{\partial \phi_{l}} c_{j k} g_{l k}
\end{aligned}
$$

and the second moment is evaluated as

$$
K_{i l}=\left.\lim _{\Delta t \rightarrow 0} \frac{\left\langle\Delta \phi_{i} \Delta \phi_{l}\right\rangle}{\Delta t}\right|_{\phi(t) \rightarrow \phi}=2 g_{i j} c_{j k} g_{l k} .
$$

Since the process $\Delta W_{i}(t)$ is Gaussian, all moments beyond $n \geq 3$ are zero, and then we get exactly a FokkerPlanck equation for $P(\{\phi\}, t)$, which can be written for an arbitrary interpretation as

$$
\begin{aligned}
\frac{\partial P}{\partial t}= & -\sum_{i} \frac{\partial}{\partial \phi_{i}}\left[f_{i}+2 \alpha \sum_{j k l} \frac{\partial g_{i j}}{\partial \phi_{l}} c_{j k} g_{l k}\right] P \\
& +\sum_{i j k l} \frac{\partial}{\partial \phi_{i}} \frac{\partial}{\partial \phi_{l}} g_{i j} c_{j k} g_{l k} P .
\end{aligned}
$$

The Fokker-Planck equation is very difficult to solve even in simple models. Nevertheless, important and experimentally observable information can be obtained from the statistical moments and correlations of the field. Moreover, quite often, approximate analytical techniques can be easily implemented for the moments. 
We now show how to derive the dynamical equation for the first moment of the field. The time derivative of this quantity can be written as

$$
\frac{d}{d t}\left\langle\phi_{i}(t)\right\rangle=\int\left\{d \phi_{j}\right\} \phi_{i} \frac{\partial P}{\partial t} .
$$

Using the Fokker-Planck equation (44) and integrating by parts,

$$
\frac{d}{d t}\left\langle\phi_{i}(t)\right\rangle=\left\langle f_{i}(\{\phi\})\right\rangle+2 \alpha \sum_{j k l}\left\langle\left(\frac{\partial g_{i j}}{\partial \phi_{l}}\right) c_{j k} g_{l k}\right\rangle .
$$

This expression will be frequently used throughout this review. The evolution of higher-order moments $\left\langle\phi_{i}^{n}(t)\right\rangle$, or of the correlation function $\left\langle\phi_{i}(t) \phi_{j}(t)\right\rangle$, can also be derived following the technique above.

Unless otherwise indicated explicitly, in this review we work in the Stratonovich interpretation $(\alpha=1 / 2)$. Moreover, it is worth emphasizing here that when the noise is affected by spatial derivative operators, the correct prescription is to take symmetric expressions of those derivatives, in order to have a well-defined continuous limit (García-Ojalvo and Sancho, 1999).

For time-correlated noise, the technique to obtain an approximate Fokker-Planck equation relies on an expansion in the correlation time of the noise (GarcíaOjalvo and Sancho, 1999; Santos and Sancho, 2001).

\section{B. Practical methodology}

We now describe a series of practical methods to evaluate relevant and experimentally accessible information from spatially extended noisy systems. Explicit examples of the application of these different methods will be given in the rest of the review.

\section{Mean-field approximation}

What in this context is known as the mean-field approximation (MFA), to be defined in what follows, constitutes a standard and easy to implement technique that provides reliable qualitative results for spatially extended systems. It is very useful to predict phase transitions, both in equilibrium and out of equilibrium. It is more powerful than simple stability analysis, and can be employed for systems with constraints, such as conserved order parameter models. Nevertheless, it does not give accurate quantitative information; for example, it does not predict correctly either the position of the transition points or the true values of the critical exponents.

The MFA can be applied to SPDEs or to FokkerPlanck equations, both approaches leading to the same results. Here we present the fundamental steps in the use of this approximation as applies to a SPDE.

As a matter of choice, consider a generic reactiondiffusion model with additive white noise,

$$
\frac{\partial \phi}{\partial t}=f(\phi)+\mathcal{D} \nabla^{2} \phi+\xi(\mathbf{r}, t) .
$$

Multiplicative noise can be considered as well and several models will be given later. Equation (47) can be written in a lattice if the Laplacian operator is discretized as

$$
\nabla^{2} \phi(x, t)=\sum_{j} \nabla_{i j}^{2} \phi_{j}(t) \equiv \frac{1}{\Delta x^{2}} \sum_{j \in \operatorname{nn}(i)}\left(\phi_{j}-\phi_{i}\right),
$$

where $\mathrm{nn}(i)$ denotes the set of $2 d$ nearest neighbors of cell $i$.

The MFA is implemented via the following assumption:

$$
\sum_{j} \nabla_{i j}^{2} \phi_{j}(t)=\frac{2 d}{\Delta x^{2}}\left(\langle\phi\rangle-\phi_{i}\right) .
$$

In that way, the MFA looks for uniform solutions of the field, $\phi=\langle\phi\rangle$, by neglecting its local fluctuations, $\Sigma_{j \in \operatorname{nn}(i)}\left(\phi_{j}-\langle\phi\rangle\right) \approx 0$.

In a $d$-dimensional lattice of cell size $\Delta x$, the model given by Eq. (47) under assumption (49) reduces to

$$
\frac{d \phi_{i}(t)}{d t}=f\left(\phi_{i}\right)+\frac{2 d \mathcal{D}}{\Delta x^{2}}\left(\langle\phi\rangle-\phi_{i}\right)+\xi_{i}(t) .
$$

The SPDE has been thus reduced to a set of uncoupled SDEs.

The MF equation (50) becomes exact for situations in which the field at each site interacts with all other sites (i.e., global coupling) in the thermodynamic limit, $N$ $\rightarrow \infty$. Therefore, the MFA applied to a system with $N$ variables and with nearest-neighbor coupling amounts to studying an infinite system with global coupling.

The next step is evaluation of the quantity $\langle\phi\rangle$, which is interpreted as the first statistical moment of the field, and accordingly it is defined as

$$
\langle\phi\rangle=F(\langle\phi\rangle) \equiv \int d \phi \phi P(\phi,\langle\phi\rangle, t),
$$

where $P(\phi,\langle\phi\rangle, t)$ is the probability distribution, which obeys the Fokker-Planck equation associated with Eq. (50),

$$
\frac{\partial P}{\partial t}=\frac{\partial}{\partial \phi}\left[-f(\phi)-\frac{2 d \mathcal{D}}{\Delta x^{2}}(\langle\phi\rangle-\phi)+\frac{\sigma^{2}}{\Delta x^{d}} \frac{\partial}{\partial \phi}\right] P .
$$

Assuming no probability flux at the boundaries, the steady-state solution of this equation reads

$$
P_{\mathrm{st}}(\phi,\langle\phi\rangle)=\mathcal{N} \exp \left[\int d \phi^{\prime} \frac{f\left(\phi^{\prime}\right)+\left(2 d \mathcal{D} / \Delta x^{2}\right)\left(\langle\phi\rangle-\phi^{\prime}\right)}{\sigma^{2} / \Delta x^{d}}\right],
$$

where $\mathcal{N}$ is a normalization constant.

Equation (51) is a self-consistency equation, since the probability distribution depends itself on the unknown average $\langle\phi\rangle$. Hence, solving the MFA is reduced to finding the set of solutions of this self-consistency equation. For those systems with $\langle\phi\rangle$ as its order parameter, the 
MFA can make predictions on the possible existence of a phase transition. In fact, one can calculate $\langle\phi\rangle$ as a function of a control parameter. A phase transition occurs, for instance, when the system leaves the state $\langle\phi\rangle$ $=0$, corresponding to a disordered phase, to reach a state $\langle\phi\rangle \neq 0$, representing an ordered phase.

Although Eq. (51) has to be integrated numerically, some analytical results can be easily obtained. For example, in systems with symmetry $\phi \rightarrow-\phi$, the function $F(\langle\phi\rangle)$ satisfies $F(\langle\phi\rangle)=-F(-\langle\phi\rangle)$ and thus $\langle\phi\rangle=0$ is always a solution of Eq. (51). By studying the Taylor expansion of $F(\langle\phi\rangle)$ at $\langle\phi\rangle \rightarrow 0$,

$$
F(\langle\phi\rangle)=a_{1}\langle\phi\rangle+a_{3}\langle\phi\rangle^{3}+O\left(\langle\phi\rangle^{5}\right),
$$

it is possible to know if the system presents a phase transition and if this transition is continuous or discontinuous. The following three cases are worthwhile to explore.

- If $a_{1}<1$ and $a_{3}<0$ in all parameter space, then $F(\langle\phi\rangle)<\langle\phi\rangle$ and the only solution of the selfconsistency equation is $\langle\phi\rangle=0$. Thus the system does not present a phase transition.

- If $a_{1}<1$ but $a_{3}>0$ in some range of parameter space, $F(\langle\phi\rangle)=\langle\phi\rangle$ has two nonzero solutions (and their symmetric values). This behavior corresponds to a discontinuous, or first-order, phase transition, for which there are three possible states (and the symmetric ones), but only two of them are stable.

- Finally, if $a_{1}>1$ in some range of parameter space, $F(\langle\phi\rangle)=\langle\phi\rangle$ has only one nonzero solution (and its symmetric value). In this case, the system presents a continuous or second-order phase transition, for which $\langle\phi\rangle$ changes continuously with the control parameter from a null value to a finite nonzero value of the field.

Figures 11(a) and 11(b) show how to find these solutions graphically. Figure 11(a) corresponds to a continuous phase transition, while Fig. 11(b) depicts a discontinuous one, in which the empty square is the unstable solution and the black squares are the stable states.

The extension of the MFA presented here to models with multiplicative noise is straightforward (Van den Broeck, Parrondo, Armero, and Hernández-Machado, 1994), but recent improvements to cover more complicated models and situations can be found in Muñoz et al. (2005).

The MFA is very useful for predicting the existence of phase transitions (and usually their type), but the location of the transition points, as noted above, is notoriously inaccurate. Several attempts have been made to improve the quantitative accuracy of the method. We briefly mention one such attempt, which does not imply any extra analytical effort and avoids spurious changes in the transition type (continuous or discontinuous).

Consider the neighborhood of a given cell $i$, as shown in Fig. 12. The average of its $2 d$ nearest neighbors is assumed to take a value $A_{i}$,
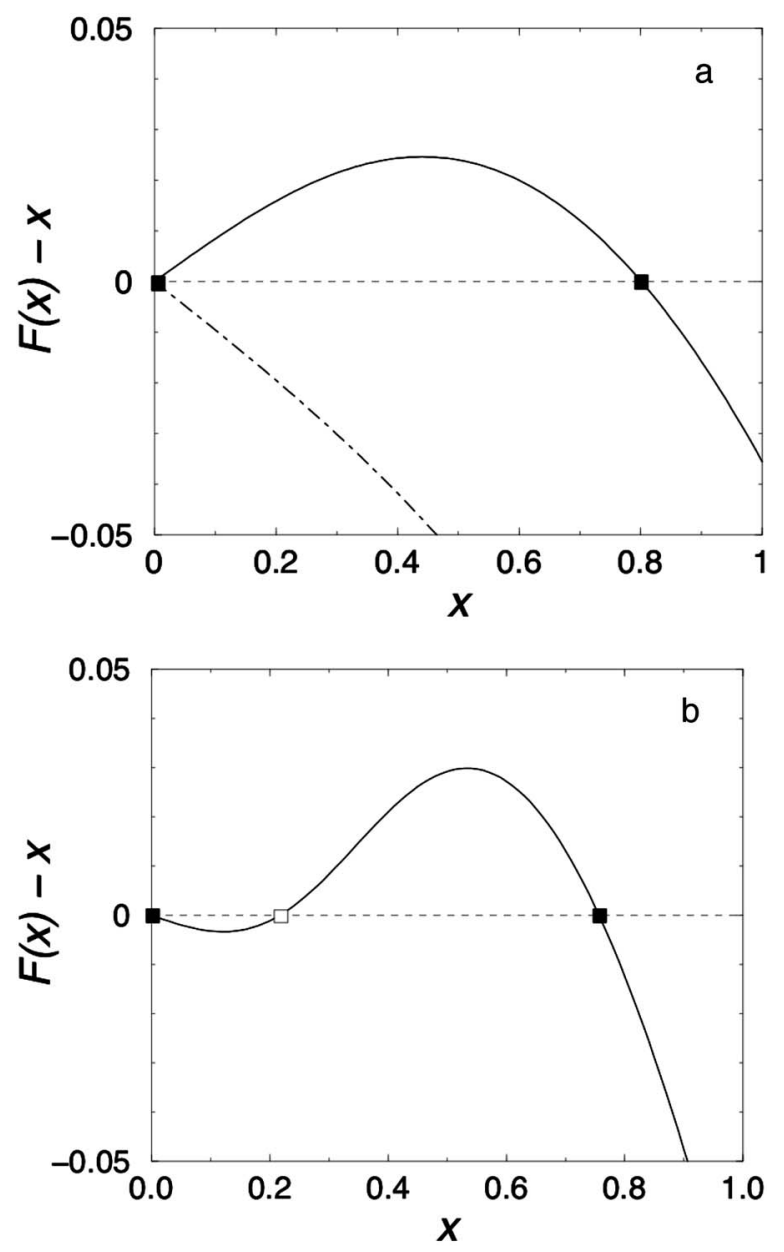

FIG. 11. Solutions (indicated by squares) of the selfconsistency equation (51). The continuous and dot-dashed lines correspond to the curve $F(x)-x$, where $F(x)$ $=\int d \phi \phi P_{\mathrm{st}}(\phi, x)$, for different values of a control parameter at both sides of a phase-transition point. Solutions of the selfconsistency equation are found for $F(x)-x=0$. (a) Continuous phase transition. For the dot-dashed curve, the unique solution is $x=0$ (stable), whereas the continuous curve intersects twice the zero line at $x=0$ (unstable solution) and $x \neq 0$ (stable solution). (b) Discontinuous phase transition with an unstable solution (empty square) and two stable ones (black squares). From Ibañes, 2001.

$$
\frac{1}{2 d} \sum_{j \in \mathrm{nn}(i)} \phi_{j}=A_{i}
$$

which, instead of being equal to the mean field $\langle\phi\rangle$ as before, is taken to result from an interpolation between that mean field and the value of the field $\phi_{i}$ itself (Ibañes, 2001),

$$
A_{i}=\frac{1}{2}\left(\phi_{i}+\langle\phi\rangle\right)
$$

This assumption is another step further in evaluating the influence of the neighboring cells. It can be written in a more standard form (Van den Broeck, Parrondo, and Toral, 1994), 


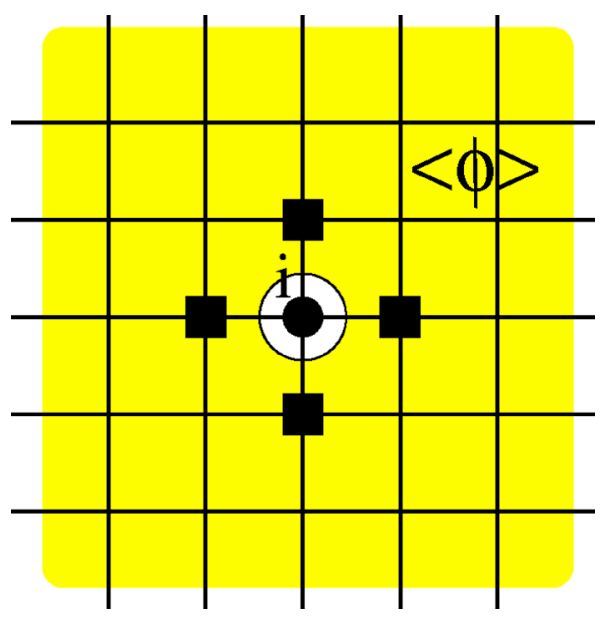

FIG. 12. (Color online) Representation of a two-dimensional square lattice, where each site (the black circle, for example) interacts with its four nearest-neighbor sites (the black squares). The MFA can be improved by replacing the field at the black squares by a new field $A_{j}$ that interpolates between $\phi_{i}$ and $\langle\phi\rangle$. From Ibañes, 2001.

$$
A_{i}=\langle\phi\rangle+c\left(\phi_{i}-\langle\phi\rangle\right)
$$

where the standard MFA is represented by $c=0$, whereas $c=1 / 2$ corresponds to the interpolation (56).

Using definition (55) together with assumption (57) in expression (48) shows that the discrete version of Eq. (47) can be written as in Eq. (50), but with an effective diffusion coefficient given by

$$
\mathcal{D}_{\text {eff }}=\mathcal{D}(1-c) \text {. }
$$

In this way, the self-consistency relation (51) is still valid in this generalized approach, provided the effective diffusion coefficient is used. In the simple case $c=1 / 2$ the intensity of the spatial coupling $\mathcal{D}$ is reduced by a factor $1 / 2$, which indicates that the standard MFA overestimates the effect of $\mathcal{D}$.

More elaborate approaches could be equally designed, taking into account, for instance, the dependence of $c$ on the noise intensities, and probably on the statistical moments. Nevertheless, the simple assumption $c$ $=1 / 2$ gives a noticeable quantitative improvement. As an example, consider the Ginzburg-Landau model with additive noise, Eq. (3). This system exhibits a phase transition (see Sec. I.B.2) that can be studied using the standard mean-field approximation, yielding the result plotted as a dashed line in Fig. 13. According to the standard MFA, as the noise intensity increases the system undergoes a phase transition from order to disorder at $\sigma_{c}^{2}$ $\approx 0.76$, which is twice the best known numerical value. This result is improved if mean field is applied with $\mathcal{D}_{\text {eff }}=\mathcal{D} / 2$ (solid line in Fig. 13). The critical point predicted by this improved mean-field analysis is $\sigma_{c}^{2} \approx 0.41$, much closer to the real value than the estimation obtained from the standard mean-field approximation. It should be noted that the evaluation of critical exponents cannot be improved with this approach, which still leads to classical mean-field values of these quantities.

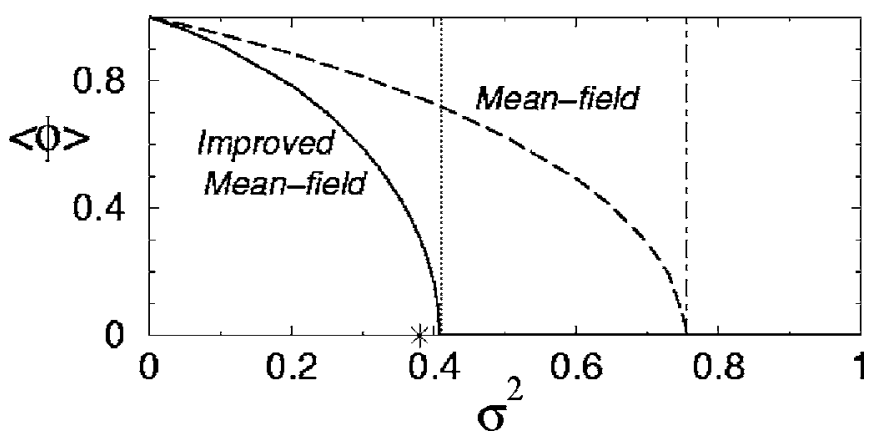

FIG. 13. Order parameter behavior for fixed $\mathcal{D}=2$ and increasing $\sigma^{2}$, for the Ginzburg-Landau model with additive noise Eq. (3) $(d=2)$. The continuous line corresponds to the improved mean-field approach, and the dashed line to the standard one. A star denotes the critical point obtained from numerical simulations of Toral and Chakrabarti (1990). Parameter values: $a=b=1 / 2$. Adapted from Ibañes, 2001.

Other approaches aimed at improving the MFA have been developed based on Eq. (57). Van den Broeck, Parrondo, and Toral (1994), for instance, introduced a correlation function approach (CFA) in which $c$ is related to the spatial correlation of the field at site $i$ with its nearest neighbors, which has to be evaluated self-consistently as well. The location of critical points is also slightly improved, but at the cost of much more involved mathematical calculations [see Van den Broeck et al. (1997) for details].

\section{Linear and nonlinear short-time analysis}

The location of transitions between a disordered $[\phi(x, t)=0]$ and an ordered $[\phi(x, t) \neq 0]$ phase can be established by analyzing the stability of the former. When necessary, the spatial character of the perturbations can be taken into account by working in Fourier space (García-Ojalvo et al., 1993).

Consider the following SPDE with both additive and multiplicative noises:

$$
\frac{\partial \phi}{\partial t}=f(\phi)+g(\phi) \eta(x, t)+\mathcal{D} \nabla^{2} \phi+\xi(x, t) .
$$

The internal additive noise $\xi(x, t)$ is Gaussian and white in both space and time, with zero mean and correlations given by Eq. (29). The external multiplicative noise $\eta(x, t)$ is Gaussian, with zero mean and correlation,

$$
\left\langle\eta(x, t) \eta\left(x^{\prime}, t^{\prime}\right)\right\rangle=2 c\left(\left|x-x^{\prime}\right|\right) \delta\left(t-t^{\prime}\right) .
$$

We want to know the initial evolution of the mean value $\langle\phi(t)\rangle$, starting from a homogeneous initial state $\langle\phi(0)\rangle=0$. Neglecting for the moment all spatial fluctuations or inhomogeneities and taking statistical averages over Eq. (59), one finds

$$
\frac{d\langle\phi\rangle}{d t}=\langle f(\phi)\rangle+c(0)\left\langle g(\phi) g^{\prime}(\phi)\right\rangle,
$$

where we used Eq. (46) or Novikov's theorem (Novikov, 1965). 
In the very early stages of the initial evolution, one can discard fluctuations around the mean value $\langle\phi(t)\rangle$, so that $\langle f(\phi)\rangle \approx f(\langle\phi\rangle)$ for any function $f$. Then Eq. (61) can be approximated by

$$
\begin{aligned}
\frac{d\langle\phi\rangle}{d t} & \approx f(\langle\phi\rangle)+c(0) g(\langle\phi\rangle) g^{\prime}(\langle\phi\rangle) \\
& \equiv f_{\text {eff }}(\langle\phi\rangle) \equiv-U_{\text {eff }}^{\prime}(\langle\phi\rangle) .
\end{aligned}
$$

Given this result, a simple analysis, either linear or nonlinear, of the effective force $f_{\text {eff }}$ or the effective potential $U_{\text {eff }}$ reveals the stability characteristics of the initial homogeneous state. In particular, $f_{\text {eff }}(0)=0$ and the sign of $U_{\mathrm{eff}}^{\prime \prime}(0)$, or of higher-order derivatives, determines the kind of transition that takes place. Beyond the transition, once the system starts to evolve from the (unstable) disordered initial state, the diffusive coupling between neighbors is able to trap the system in a new steady state, homogeneous or not (Van den Broeck, Parrondo, and Toral, 1994). We come back to these arguments in Sec. III.

\section{Noise-effective models}

In the previous paragraphs, we have seen that the main effects of multiplicative noise can be incorporated as a modification of the deterministic force. The approach was limited to studying the stability of an initial homogeneous state during the very early stages of time evolution. In what follows, we present an extension of this idea, based on a nonstandard small noise expansion, that is valid for the whole dynamics of the system.

Consider a nonlinear reaction-diffusion equation for the field $\phi(x, t)$,

$$
\frac{\partial \phi}{\partial t}=f(\phi)+\varepsilon^{1 / 2} g(\phi) \eta(x, t)+\mathcal{D} \frac{\partial^{2} \phi}{\partial x^{2}},
$$

where $\varepsilon$ is an explicit measure of the strength of the noise $\eta(x, t)$, which is assumed Gaussian of zero mean and correlation given by Eq. (60). Our goal here is to separate the two main effects of noise, namely, a systematic part and a diffusive wandering. The subtlety of the problem lies in the fact that the noise modifies simultaneously two aspects of the system's dynamics which are different in nature (systematic bias and diffusive propagation), and which cannot be naively associated with the usual separation between deterministic and stochastic forces. The key idea of our approach is that the separation of these effects is related to an actual separation of time scales within the system dynamics. On the one hand, there are fast fluctuations that produce an average motion at relatively small time scales; a temporal coarse graining would thus eliminate these fast fluctuations, leading to an averaged motion different from the deterministic one, and therefore having a different dynamics. On the other hand, residual slow fluctuations are responsible for a diffusive motion which will not be considered here [see Rocco et al. (2002) for an analytical study].
To be more explicit we illustrate this approach in relation with Eq. (63). A crucial feature of multiplicative noise in the Stratonovich interpretation is that the mean value of the noise term in the Langevin equation is nonzero, even though the noise itself has zero mean. This produces the so-called spurious drift. As discussed in the previous section, this mean value can be evaluated from Eq. (46), or using Novikov's theorem for Gaussian noises (Novikov, 1965), and gives

$$
\varepsilon^{1 / 2}\langle g(\phi) \eta(x, t)\rangle=\varepsilon c(0)\left\langle g^{\prime}(\phi) g(\phi)\right\rangle .
$$

According to this result, Eq. (63) can be rewritten in a more useful form,

$$
\frac{\partial \phi}{\partial t}=h(\phi)+\varepsilon^{1 / 2} R(\phi, x, t)+\mathcal{D} \frac{\partial^{2} \phi}{\partial x^{2}},
$$

in terms of a new reaction term,

$$
h(\phi) \equiv f(\phi)+\varepsilon c(0) g^{\prime}(\phi) g(\phi),
$$

and a new stochastic force defined as

$$
R(\phi, x, t) \equiv g(\phi) \eta(x, t)-\varepsilon^{1 / 2} c(0) g^{\prime}(\phi) g(\phi),
$$

which has zero mean and correlation,

$$
\begin{aligned}
\left\langle R(\phi, x, t) R\left(\phi, x^{\prime}, t^{\prime}\right)\right\rangle= & \left\langle\phi(x, t) \eta(x, t) \phi\left(x^{\prime}, t^{\prime}\right) \eta\left(x^{\prime}, t^{\prime}\right)\right\rangle \\
& +O\left(\varepsilon^{1 / 2}\right) .
\end{aligned}
$$

With this rearrangement, we have separated the systematic contribution of the multiplicative noise from a residual stochastic one. This separation is useful because of the white character of the noise, which gives a simple form for the average of the multiplicative noise term (64), with no explicit time dependence. Since the new noise term $R$ has no systematic contribution left, we can proceed further discarding it, and the problem reduces to the deterministic effective model,

$$
\frac{\partial \phi}{\partial t}=f(\phi)+\varepsilon c(0) g^{\prime}(\phi) g(\phi)+\mathcal{D} \frac{\partial^{2} \phi}{\partial x^{2}} .
$$

In some cases, the resulting equation has the same form as the original deterministic one, but with different effective parameters in the reaction terms.

We will invoke this approach quite often in this review and singularly in Sec. VI.

\section{Central-moment approximation}

The short-time analysis described above gives reliable information about the position and type of a given transition point, but it fails to provide a global picture of the phase diagram of the system, as the mean-field approximation does. In particular, this analysis is not able to determine the existence of reentrant transitions. As such, a combination of short-time analysis and meanfield approximation can be developed to determine the time evolution of the central moments $\mu_{n}=\left\langle\Delta^{n}\right\rangle$, where $\Delta \equiv \phi-\langle\phi\rangle$. This approach, which incorporates most of the key points of the methods described previously, allows a description of the phase-transition scenario in terms of a dynamical-system picture (Kawai et al., 2004). 
This method, although based in the mean-field approximation, does not require knowledge of the stationary probability distribution $P_{\mathrm{st}}(\phi,\langle\phi\rangle)$, and can be extended to time-dependent problems and pattern-forming systems (Dutta et al., 2005).

For illustration purposes, consider again the reactiondiffusion model with additive noise (47). The starting point of the central-moment analysis is Eq. (50), corresponding to the MFA of the model. From this selfconsistent Langevin equation one can derive the evolution of the first moment or order parameter $\langle\phi\rangle$,

$$
\langle\dot{\phi}\rangle=\langle f(\phi)\rangle \text {. }
$$

This equation does not incorporate explicitly the diffusion parameter $\mathcal{D}$. Thus we consider also the equation satisfied by the second central moment,

$$
\begin{aligned}
\dot{\mu}_{2}= & 2[\langle\phi f(\phi)\rangle-\langle\phi\rangle\langle f(\phi)\rangle] \\
& +\frac{4 d \mathcal{D}}{\Delta x^{2}}\left(\langle\phi\rangle^{2}-\left\langle\phi^{2}\right\rangle\right)+\frac{2 \sigma^{2}}{\Delta x^{d}} .
\end{aligned}
$$

A Taylor series expansion of the nonlinear function $f(\phi)$ appearing in Eqs. (70) and (71) introduces higher-order moments, whose dynamical equations should in turn be considered. This leads to an infinite set of equations to which a closure should be applied to render the problem tractable. In order to do that, the higher-order central moments can be approximated assuming a Gaussian-like stationary distribution. This implies that odd central moments are zero and the first even central moments are

$$
\mu_{4}=3 \mu_{2}^{2}, \quad \mu_{6}=15 \mu_{2}^{3} .
$$

Using this decoupling scheme, the two equations (70) and (71) can be solved in the steady state, and the behavior of the order parameter $\langle\phi\rangle_{\text {st }}$ as a function of control parameters such as $\mathcal{D}$ or the noise intensity $\sigma^{2}$ can be analyzed using standard techniques from dynamicalsystem theory (Kawai et al., 2004).

This method can be easily generalized to multiplicative noise in an arbitrary stochastic interpretation, or to the presence of two or more variables (Kawai et al., 2004).

\section{Numerical algorithms for white and structured noises}

Numerical integration of SPDEs is a necessary complement to the approximate analytical techniques described in the preceding subsections. In this respect, and even though first-order, Euler-type algorithms could be a reasonable choice in most cases, singularly with white noise, the systematic errors introduced by the deterministic part make it advisable to consider higherorder algorithms.

The simplest of these schemes, of second order in the deterministic part but of first order in the stochastic one, is the so-called Heun's predictor-corrector algorithm (Gard, 1987; Toral, 1995; García-Ojalvo and Sancho, 1999). It was originally derived for the Stratonovich interpretation, but we prefer to present here an extension valid for any stochastic interpretation. The predictor step is simply the first-order algorithm (39) for $\alpha=0$,

$$
\tilde{\phi}_{i}(t+\Delta t)=\phi_{i}(t)+f_{i}(\{\phi(t)\}) \Delta t+g_{i j}(\{\phi(t)\}) \mathcal{X}_{j}(t),
$$

and the corrector step incorporates the stochastic interpretation,

$$
\begin{aligned}
\phi_{i}(t+\Delta t)= & \phi_{i}(t)+\frac{f_{i}(\{\phi(t)\})+f_{i}(\{\tilde{\phi}(t)\})}{2} \Delta t \\
& +g_{i j}[(1-\alpha)\{\phi(t)\}+\alpha\{\tilde{\phi}(t)\}] \mathcal{X}_{j}(t) .
\end{aligned}
$$

In both Eqs. (73) and (74), the stochastic process $\mathcal{X}_{j}(t)$ [see Eq. (34)] is discretized as

$$
\mathcal{X}_{i}(t)=\sqrt{\frac{2 \sigma^{2} \Delta t}{\Delta x^{d}}} \beta_{i},
$$

where $\beta_{i}$ represent Gaussianly distributed, independent random numbers of zero mean and unit standard deviation. Higher-order algorithms can also be developed following classical approaches (Rao et al., 1974) or more recent ones (Mannella, 2004).

Finally, it is worth remarking that the algorithm given above is also valid for white noises in time and arbitrary correlation in space, provided the latter is implemented in the numerical generation of the process $\mathcal{X}_{j}(t)$ (see below).

Colored (nonwhite) noises in time need a more careful analysis, and incorporate a different type of numerical difficulties. The advantage in this case, however, is that stochastic interpretation problems do not arise, and generic Runge-Kutta algorithms can in principle be used. For example, a simple predictor-corrector algorithm for noise colored in time uses the predictor step discussed above [see Eq. (73)] but with a different corrector,

$$
\begin{aligned}
\phi_{i}(t+\Delta t)= & \phi_{i}(t)+\frac{f_{i}(\{\phi(t)\})+f_{i}(\{\tilde{\phi}(t)\})}{2} \Delta t \\
& +\frac{g_{i j}(\{\phi(t)\})+g_{i j}(\{\tilde{\phi}(t)\})}{2} \mathcal{X}_{j}(t),
\end{aligned}
$$

where now $\mathcal{X}_{j}(t)$ stands for a colored stochastic process which has to be generated according to the prescribed properties of the noise. This is the point that we discuss in what follows.

Assume that the Gaussian noise we want to generate has zero mean and a correlation given by

$$
\left\langle\eta(x, t) \eta\left(x^{\prime}, t^{\prime}\right)\right\rangle=c\left(\left|x-x^{\prime}\right|\right) \gamma\left(\left|t-t^{\prime}\right|\right),
$$

where the spatial correlation function $c(r)$ will be assumed to be, for instance,

$$
c(r)=\frac{\sigma_{0}^{2}}{(\sqrt{2 \pi})^{d} \lambda^{d}} e^{-r^{2} / 2 \lambda^{2}} .
$$

Other possible spatial correlation functions can be easily implemented. For the temporal part one can take the well-known Ornstein-Uhlenbeck form, 


$$
\gamma(s)=\frac{1}{\tau} e^{-s / \tau}
$$

This type of noise, easily implemented and incorporating relevant ingredients for practical purposes, has three independent parameters: the intensity $\sigma_{0}^{2}$, the correlation length $\lambda$, and the correlation time $\tau$, and two correlation functionals $c(r)$ and $\gamma(t)$. More complicated prescriptions can be proposed to describe highly structured spatiotemporal noises, as, for instance, to emulate turbulent flows (Martí et al., 1997) within the approach known as synthetic turbulence.

The method is particularly well adapted to noises with simple spatial correlation in Fourier space. For the correlation function (78) one obtains

$$
c(k)=\int_{\mathcal{R}} d x^{d} c(r) e^{i k r}=\sigma_{0}^{2} e^{-\lambda^{2} k^{2} / 2}
$$

so that the complete noise correlation (77) in Fourier space is

$$
\left\langle\eta(k, t) \eta\left(k^{\prime}, t^{\prime}\right)\right\rangle=\sigma_{0}^{2} e^{-\lambda^{2} k^{2} / 2} \delta^{d}\left(k+k^{\prime}\right) \frac{e^{-\left|t-t^{\prime}\right| / \tau}}{\tau} .
$$

This noise obeys the dynamical equation

$$
\tau \frac{d \eta}{d t}=-\eta+\xi(k, t),
$$

where $\xi(k, t)$ is a Gaussian noise white in time, and whose spatial properties have to be chosen adequately according to Eq. (80).

Equation (82) is a linear equation, and an exact solution can be obtained for any integration step $\Delta t$ (GarcíaOjalvo and Sancho, 1999),

$$
\eta(k, t+\Delta t)=\eta(k, t) e^{-\Delta t / \tau}+\sqrt{\frac{1-e^{-2 \Delta t / \tau}}{\tau}} \alpha(k, t),
$$

where the random process $\alpha(k, t)$ is constructed as

$$
\alpha(k, t)=c(k)^{1 / 2} \beta(k, t),
$$

where $\beta(k, t)$ is a Gaussian white noise in Fourier space with correlation,

$$
\left\langle\beta(k, t) \beta\left(k^{\prime}, t^{\prime}\right)\right\rangle=2 \delta\left(t-t^{\prime}\right) \delta^{d}\left(k+k^{\prime}\right) .
$$

This anticorrelated process can be generated using standard procedures (García-Ojalvo et al., 1992).

If we choose the initial values of $\eta(k, 0)$ to be Gaussian distributed with correlation,

$$
\left\langle\eta(k, 0) \eta\left(k^{\prime}, 0\right)\right\rangle=\frac{\sigma_{0}^{2}}{\tau} e^{-\lambda^{2} k^{2} / 2} \delta^{d}\left(k+k^{\prime}\right),
$$

then the noise $\eta(k, t)$ is stationary, isotropic, and Gaussian with correlation (81).

The numerical algorithm is then implemented as follows. At each integration time step the noise $\eta(k, t)$ is generated and Fourier antitransformed, to get $\eta(x, t)$ or $\eta_{i}(t)$ in the lattice. Then the quantity $\mathcal{X}_{i}(t)$, needed in algorithm (76), is obtained through

$$
\mathcal{X}_{i}(t)=\int_{t}^{t+\Delta t} d t^{\prime} \eta_{i}\left(t^{\prime}\right)=\eta_{i}(t) \Delta t .
$$

Other types of noise can be generated following these lines, or by more specific methods (Makse et al., 1996; García-Ojalvo and Sancho, 1999; Romero and Sancho, 1999; Santos and Sancho, 2001).

\section{NONEQUILIBRIUM PHASE TRANSITIONS}

As commented on in the Introduction, see Sec. I.B.2, phase transitions are one of the most important examples of cooperative behavior in macroscopic systems. Equilibrium phase transitions are well described with the methodologies of thermodynamics and statistical mechanics. From these disciplines, it is known that an increase in temperature (or internal fluctuations) produces, in the majority of cases, a disordering effect that manifests itself in the form of a phase transition, continuous or discontinuous, from an ordered macroscopic phase to a less ordered one. This section focuses in the opposite situation, when an increase in the intensity of an external noise, mainly multiplicative, can produce nonequilibrium phase transitions, of both first and second order, from a macroscopic disordered phase to a more ordered one.

We also discuss in what follows the dynamics associated with this type of transition. Specifically, we examine two different situations that appear when the system evolves from an initial unstable phase toward a final, more stable phase. The first scenario consists in the development of fronts of the stable phase as it invades the unstable one. In the second case, we study the intermediate time regime with two equally stable phases competing with each other. This phenomenon presents universal scaling characteristics. Finally, we discuss the role of spatiotemporally structured noise versus white noise.

\section{A. Continuous phase transitions}

There are many examples in the literature showing that noise can induce nonequilibrium second-order, or continuous, phase transitions. Two different physical mechanisms can be responsible for these transitions: (i) a short-time instability (linear or nonlinear) produced by a multiplicative noise, which leads to a phase that is stabilized by the spatial coupling (Becker and Kramer, 1994; Van den Broeck, Parrondo, and Toral, 1994); and (ii) an entropic mechanism appearing at long times, which is reflected in the occurrence of qualitative changes in the steady-state probability distribution of the system (Ibañes et al., 2001).

In the first mechanism, spatial coupling plays an essential role (i.e., the phase transition would not exist without it); the second mechanism is an extension to macroscopic phases of noise-induced transitions known to exist in nonspatial dynamical systems (Horsthemke and Lefever, 1984). We also review here other situations, such as those where the additive noise is the driving force beneath the ordering phase transition (provided a 
multiplicative noise is present) (Landa et al., 1998), and the case in which a purely multiplicative noise produces an absorbing transition with a specific nonequilibrium universality class (Grinstein et al., 1996).

Noise-induced phase transitions can be characterized analogously to the equilibrium case. Given a field variable $\phi(\mathbf{x}, t)$, one can define a macroscopic order parameter as

$$
M(t)=\int_{V} \phi(\mathbf{x}, t) d V
$$

An intensive version of this order parameter and a generalized susceptibility are defined accordingly,

$$
m=\frac{M}{V}, \quad \chi=\frac{\left\langle M^{2}\right\rangle-\langle M\rangle^{2}}{\mathcal{N}}
$$

where the brackets denote temporal averages computed in the stationary state. $V$ is the $d$-volume of the system and $\mathcal{N}$ is a parameter representing the intensity of the external noise (as $k_{B} T$ does in equilibrium), whose exact value depends on the model under study. The singular behavior of $m$ and $\chi$ as a function of a given control parameter indicates the occurrence of a phase transition, which can be characterized, analogously to equilibrium, by nonequilibrium critical exponents $\beta$ and $\gamma$,

$$
m \sim\left|\alpha-\alpha_{c}\right|^{\beta}, \quad \chi \sim\left|\alpha-\alpha_{c}\right|^{-\gamma},
$$

where $\alpha$ is the control parameter of the transition and $\alpha_{c}$ is its critical value.

\section{Short-time instabilities}

We consider the Ginzburg-Landau model (3) with $b=1$ and an extra multiplicative noise term,

$$
\frac{\partial \phi}{\partial t}=[-a+\eta(\mathbf{x}, t)] \phi-\phi^{3}+\mathcal{D} \nabla^{2} \phi+\xi(\mathbf{x}, t) .
$$

The additive noise $\xi(\mathbf{x}, t)$ is assumed to be white in space and time, whereas the multiplicative external noise is assumed to be only white in time. Respective correlations are given by the $d$-dimensional versions of Eqs. (29) and (60). This model presents a homogeneous steady state $\langle\phi\rangle_{\mathrm{st}}=0$, which is stable in the absence of multiplicative noise, provided $a>0$.
The short-time analysis Eqs. (61) and (62) applied to Eq. (91) gives

$$
\frac{d\langle\phi\rangle}{d t} \sim[-a+c(0)]\langle\phi\rangle-\langle\phi\rangle^{3} .
$$

This equation shows that the homogeneous state $\langle\phi\rangle_{\text {st }}$ $=0$ becomes linearly unstable when the noise intensity increases beyond $c(0)=a$. We note that in the absence of spatial coupling (and ignoring the additive noise for the moment), the stationary probability distribution, as obtained from the corresponding version of the FokkerPlanck equation (23), reads

$$
P_{\mathrm{st}}(\phi)=\phi^{-1-a / c(0)} \exp \left(-\frac{\phi^{2}}{2 c(0)}\right)
$$

This probability distribution has its maximum at $\phi_{\max }$ $=0$ and is monotonically decreasing for any noise intensity $c(0)$. Therefore, in the absence of spatial coupling, the noise-induced short-time instability established by Eq. (92) is not maintained for long times. The situation changes in the presence of spatial coupling, since interaction between neighbors is able to keep the system for long times in the noise-induced "minimum" of the shorttime potential [see Eq. (62)] corresponding to Eq. (92), giving rise to a phase transition (Van den Broeck, Parrondo, and Toral, 1994). This ordering transition is shown in Fig. 14 (top plot), where it can be identified by the small left peak in the susceptibility, which corresponds to a divergence in the thermodynamic limit.

As shown in the bottom plot of Fig. 14, mean-field calculations indicate that there is also a second transition from order back to disorder for higher noise intensities (García-Ojalvo et al., 1996). Such reentrant transition is also found numerically in $d=2$, as shown in the top plot of Fig. 14. A finite-size scaling analysis of both phase transitions shows that the critical exponents are those of the equilibrium Ising universality class (GarcíaOjalvo and Sancho, 1999).

In summary, we have seen so far that noise-induced phase transitions arise when external noise produces a short-time instability, taking the system to a noiseinduced short-lived state that is then stabilized by the spatial coupling. Thus the diffusion parameter $\mathcal{D}$ is totally necessary. This mechanism was first reported in a different model, where the reaction term and multiplicative function (59) are given by

$$
f(\phi)=-\phi\left(1+\phi^{2}\right)^{2}, \quad g(\phi)=1+\phi^{2}, \quad \xi=0 .
$$

This system also exhibits an ordering and a reentrant transition (Van den Broeck, Parrondo, and Toral, 1994) and the same equilibrium universality class (Van den Broeck et al., 1997).

In the previous two models (91) and (94), the shorttime instability leading to the phase transition was 

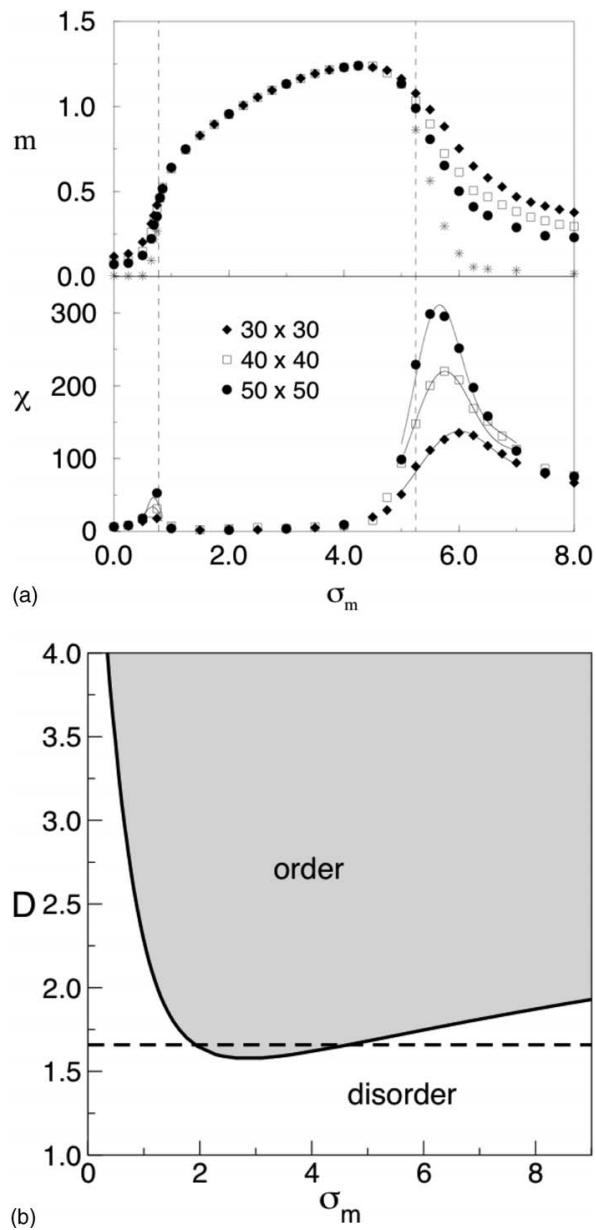

FIG. 14. Noise-induced second-order phase transition. Top: Order parameter $m$ and susceptibility $\chi$ versus multiplicative noise intensity [denoted here as $\sigma_{m}^{2}$, corresponding to $c(0)$ in the text] in model (91), for different lattice sizes $(d=2)$. Starred symbols in the upper figure are an extrapolation to infinite size, and vertical lines are estimates of the two critical points. Bottom: Mean-field calculation showing the existence of two consecutive phase transitions for increasing noise intensity (an example is given by the horizontal dashed line). The solid line denotes the transition boundary, as obtained from the selfconsistency equation (51), between regions with $m=0$ (disorder) and $m \neq 0$ (order). Parameter values: $a=-0.75$ and $\sigma^{2}$ $=1.0$ for both plots and $\mathcal{D}=3.0$ for the top plot. Note how the mean-field analysis in the bottom plot overestimates the size of the ordered region, as obtained from numerical results of the full model, shown in the top plot. Adapted from García-Ojalvo et al., 1996.

linear. But there are also phase transitions associated with a nonlinear instability. Consider, for instance, the following model (Carrillo et al., 2002):

$$
\frac{\partial \phi}{\partial t}=-\left(1+\phi^{2}\right) \phi^{3}+\phi^{2} \eta(\mathbf{x}, t)+\mathcal{D} \nabla^{2} \phi+\xi(\mathbf{x}, t) .
$$

The short-time analysis leads to an effective force, as defined in Eq. (62), given by $f_{\text {eff }}=[2 c(0)-1] \phi^{3}-\phi^{5}$, which exhibits a transition point at a critical value $c(0)=0.5$, corresponding to a nonlinear instability of the homogeneous zero state. Again for larger noise intensities, a second or reentrant transition arises. Mean-field calculations and numerical simulations confirm this scenario, which is very similar to that of the former GinzburgLandau model.

\section{Entropic mechanism and long-time analysis}

Continuous phase transitions can be induced by a different mechanism, which relies on the long-term behavior of the local dynamics. This mechanism can be explained from the (homogeneous) stationary probability distribution of the system, and does not involve any short-time instability (Ibañes et al., 2001). The resulting so-called entropy-driven phase transition (EDPT) is the natural extension of the well-known noise-induced transitions in nonspatial dynamical systems (Horsthemke and Lefever, 1984). EDPTs share some characteristics with the so-called lower critical solution temperature (LCST) transitions, which arise in certain polymer blends, and whose phenomenology goes in the counterintuitive direction followed in this review: by increasing the temperature the polymer system evolves from a disordered toward an ordered state (Snyder et al., 1983).

A simple explanation of the EDPT mechanism is that noise is much more intense in the homogeneous or disordered phase $\phi=0$ than in an ordered region with $\phi$ $\neq 0$. Then the noise drives the system far from $\phi=0$ and the spatial coupling fixes it at $\phi \neq 0$. This fact can be controlled by the noise coupling function, as shown in what follows. A generic model exhibiting an EDPT has the following structure:

$$
\frac{\partial \phi}{\partial t}=-\Gamma(\phi) \frac{\delta \mathcal{F}}{\delta \phi}+\Gamma(\phi)^{1 / 2} \eta(\mathbf{x}, t),
$$

which describes a relaxational dynamics in a free-energy functional $\mathcal{F}(\{\phi\})$ with a field-dependent kinetic coefficient $\Gamma(\phi)$. A comprehensive study of the different possibilities and phenomenologies of this generic model has been presented by Buceta and Lindenberg (2004). These types of kinetic coefficients appear in coarse-grained field equations (Langer, 1971; Ramírez-Piscina et al., 1993) and, in general, they are large in the disordered phase and small in the ordered one (Kitahara and Imada, 1978; Martin, 1990). Accordingly, we consider a kinetic coefficient of the form

$$
\Gamma(\phi)=\frac{1}{1+c \phi^{2}} .
$$

We assume a free energy of the form

$$
\mathcal{F}=\int d^{d} x\left[V(\phi)+\frac{\mathcal{D}}{4 d}(\nabla \phi)^{2}\right],
$$

where $V(\phi)$ is a local potential chosen to be monostable: $V(\phi)=a \phi^{2} / 2$. Under these conditions, in the absence of noise the system relaxes to the homogeneous state $\phi$ $=0$. Assuming that $\eta(\mathbf{x}, t)$ is a white noise in space and time of intensity $\tilde{\sigma}^{2} \equiv \sigma^{2} / \Delta x^{d}$, the Fokker-Planck equation exhibits an exact steady probability distribution with a Boltzmann-like form $P(\{\phi\})_{\text {st }} \sim e^{-\mathcal{F}_{\text {eff }} / \sigma^{2}}$, where $\mathcal{F}_{\text {eff }}$ 


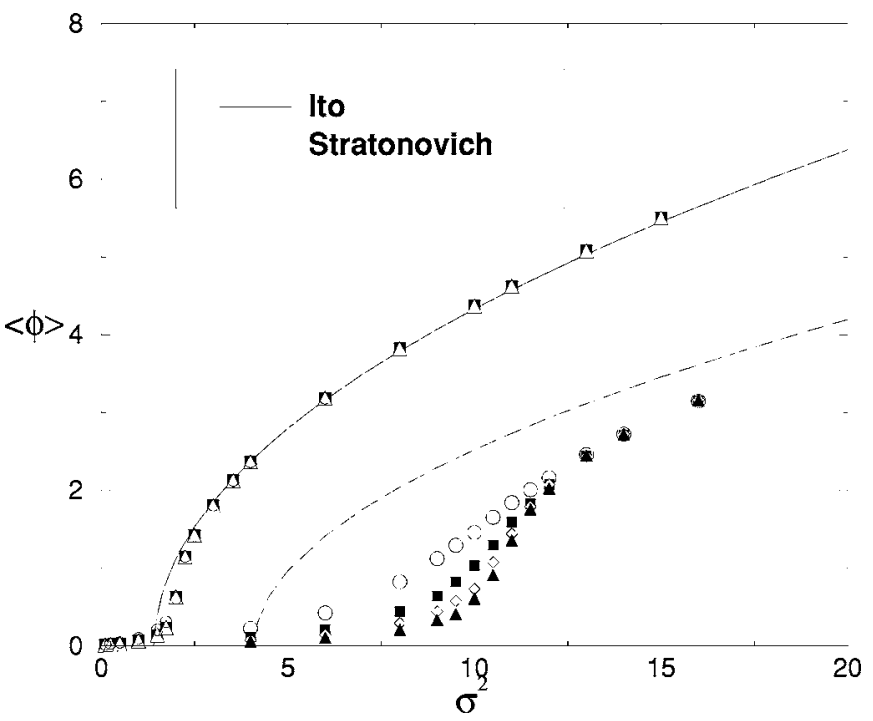

FIG. 15. Order parameter versus multiplicative (white) noise intensity for model (96). Mean-field (lines) and numerical simulation (symbols) results for the Itô (continuous line) and Stratonovich (dashed line) interpretations. Lattice sizes $(d=2)$ : $L=16$ (circles), $L=24$ (squares), and $L=32$ (triangles) for Itô. $L=64$ (triangles), $L=48$ (diamonds), $L=32$ (squares), and $L$ $=16$ (circles) for Stratonovich. Parameter values: $a=1, c=05$, and $\mathcal{D}=4$. From Carrillo et al., 2003.

is an effective free energy with a new local interaction term given by

$$
V_{\text {eff }}=\frac{a}{2} \phi^{2}+(1-\alpha) \tilde{\sigma}^{2} \ln \Gamma(\phi)^{1 / 2} .
$$

This result is valid for any stochastic interpretation, defined by the parameter $\alpha$, as described in Eq. (36).

A short-time stability analysis of this model gives

$$
\frac{\partial\langle\phi\rangle}{\partial t}=-\left(a+2 \alpha \tilde{\sigma}^{2}\right)\langle\phi\rangle+\frac{\mathcal{D}}{2 d} \nabla^{2}\langle\phi\rangle,
$$

which does not present an instability for any value of $\alpha$. On the other hand, an analysis of the maxima of the local potential (99) predicts a continuous ordering phase transition arising near $\tilde{\sigma}^{2} \sim a / 2(1-\alpha) c$, with no corresponding reentrant transition. This is confirmed by numerical simulations and mean-field calculations in $d=2$, as shown in Fig. 15. Note that the phase transition occurs not only in the Stratonovich interpretation but also in the Itô interpretation (Carrillo et al., 2003). This contrasts with noise-induced phase transitions due to shorttime instabilities, which typically arise only in the Stratonovich interpretation. Furthermore, a theoretical analysis and exhaustive numerical simulations indicate that this model belongs to the $d$-dimensional Ising universality class (Ibañes, 2001).

\section{The role of additive noise}

Usually, an increase of additive noise has a disordering effect. However, in the presence of an additional source of multiplicative noise the opposite effect may be observed. This situation has been studied by Landa et al. (1998) with model (94), with a coupling function of the form $g(\phi)=a^{2}+\phi^{2}$. In that case $a$ controls an ordering phase transition, followed by a reentrant transition back to disorder. This situation corresponds to a single noise with both additive and multiplicative contributions. However, this phenomenology is quite universal: one can take $a=0$ and add an independent additive noise, obtaining the same results. This means that a pure additive noise is able to induce both ordering and disordering phase transitions, provided a multiplicative noise of adequate intensity is present, and the diffusion parameter $\mathcal{D}$ lies in an appropriate domain (Landa et al., 1998).

\section{Multiplicative-noise universality class}

In spite of their counterintuitive nature, all noiseinduced phase transitions described so far belong to the equilibrium Ising universality class. This can be traced back to the existence of an inhomogeneous term in the corresponding SPDE, namely, the additive noise term. Models with only multiplicative noise, on the other hand, exhibit purely nonequilibrium universality classes [see Muñoz (2004) for a recent review]. Consider, for instance, the generic model

$$
\frac{\partial \phi}{\partial t}=-a \phi-\phi^{p}+\mathcal{D} \nabla^{2} \phi+\phi^{q} \eta(\mathbf{x}, t),
$$

where all parameters are positive, and the noise is interpreted in the Stratonovich sense. The exponents $p \geq 2$ (2 or 3$)$ and $q \geq 0$ (1/2 or 1$)$ define the most common situations. This model exhibits a nonequilibrium phase transition as a function of the control parameter $a$ (Grinstein et al., 1996). For $a>a_{c}$, the steady state is $\phi=0$, called an absorbing phase. For $a<a_{c}$, on the other hand, the steady state is $\langle\phi\rangle \neq 0$, and is called an active phase.

The generic equation (101) encompasses the following two important models, exhibiting two different universality classes:

- The case $p=2$ and $q=1 / 2$ corresponds to the Reggeon field theory (RFT) model of directed percolation (Cardy and Sugar, 1980; Dickman, 1994; Marro and Dickman, 1998).

- The particular case $p=3$ and $q=1$ defines what it is known as the multiplicative noise universality class (Grinstein et al., 1996). By means of the Hopf-Cole transformation $[\phi=\exp (-h)]$, the new field $h(x, t)$ follows a Kardar-Parisi-Zhang equation (Kardar et al., 1986). This model leads to an ordering transition by increasing the intensity of the white noise (Genovese et al., 1998).

These types of phase transitions can be characterized by critical exponents, both static and dynamic, which depend on the dimensionality of the space and on the relevant parameters $p$ and $q$. The different universality classes have been detailed by Muñoz (2004). 


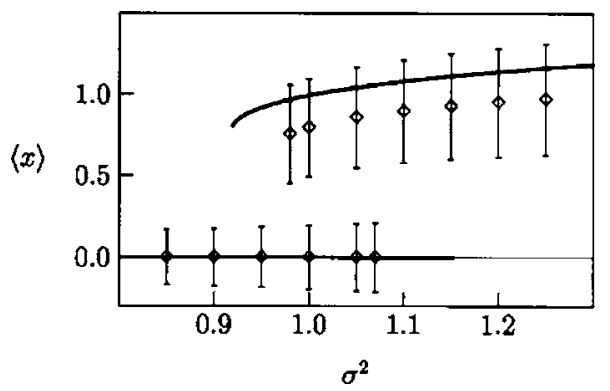

FIG. 16. Order parameter (denoted here as $\langle x\rangle$ ) versus multiplicative noise intensity [denoted here as $\sigma^{2}$, corresponding to $2 c(0)$ in the text] for model (102). Symbols are numerical simulation data and lines are the mean-field prediction. Simulations were made on a square two-dimensional lattice of $100 \times 100$ cells. From Müller et al., 1997.

\section{B. First-order phase transitions}

Noise can also induce first-order, or discontinuous, phase transitions. A model exhibiting this behavior was first introduced by Müller et al. (1997) in the form

$$
\frac{\partial \phi}{\partial t}=-a \phi+\phi^{3}-\phi^{5}+\left(1+\phi^{2}\right) \eta(\mathbf{x}, t)+\mathcal{D} \nabla^{2} \phi,
$$

where $\eta(\mathbf{x}, t)$ was assumed to be white in time with correlation (60). Applying the short-time analysis, Eq. (62), to this model leads to the effective force $f_{\text {eff }}=-[a$ $-2 c(0)] \phi+[1+2 c(0)] \phi^{3}-\phi^{5}$, which exhibits a subcritical pitchfork bifurcation with increasing noise intensity. This corresponds to a discontinuous phase transition, as shown in Fig. 16. This figure plots the mean-field and numerical simulation results of Müller et al. (1997), which display a region of coexistence between the ordered and disordered phases and hysteretic behavior, both of them characteristics of first-order phase transitions.

The same kind of transition can be obtained through a nonlinear instability, as in the following model (Carrillo et al., 2002):

$$
\frac{\partial \phi}{\partial t}=-\left(1+\phi^{4}\right) \phi+\phi^{2} \eta(\mathbf{x}, t)+\mathcal{D} \nabla^{2} \phi+\xi(\mathbf{x}, t) .
$$

Its corresponding effective force is $f_{\text {eff }}=-\phi+2 c(0) \phi^{3}$ $-\phi^{5}$, which again undergoes a subcritical bifurcation as a function of the noise intensity, with a regime of two coexisting phases. For large noise, both cases described above exhibit a reentrant continuous transition that restores the homogeneous disordered phase.

First-order nonequilibrium phase transitions induced by additive noise have been reported by Zaikin et al. (1999). In that case, the model studied was the one given by Eq. (94), with $g(\phi)=a^{2}+\phi^{2}$. There, the coexistence region (and thus the discontinuity in the phase transition) exists for large enough intensity of multiplicative noise, evidencing again the need of multiplicative noise in order to have an ordering effect of additive random fluctuations.

\section{Dynamics of nonequilibrium phase transitions}

The dynamical aspects of phase transitions constitute an important part of our knowledge of these cooperative phenomena. It is thus equally important in the context of noise-induced nonequilibrium phase transitions to establish the relevance of noise, both in the front evolution of a more stable phase invading a less stable one (Santos and Sancho, 1999) and in the dynamical scaling regime of domain growth associated with coarsening in phase separation (Gunton et al., 1983).

These dynamical behaviors can be studied in any of the models introduced in this section, either GinzburgLandau models as in Eq. (91) or the EDPT model defined by Eqs. (96) and (97).

\section{Noise-induced fronts}

The influence of multiplicative fluctuations on front dynamics has mostly been studied in models for deterministic front solutions. As representative references we refer to the articles by Frankowicz et al. (1991); Schimansky-Geier and Zulicke (1991); de Pasquale et al. (1992); Armero et al. (1996, 1998); Sancho and Sánchez (2000); Tripathy et al. (2001); and the review by Panja (2004). Additive noise has also been shown to unveil hidden nonlinearities via a ratchetlike effect (Clerc et al., 2005). Following the spirit of this review, we focus here, complementarily, on fronts that are indeed induced by multiplicative noise.

We start with a model such as that in Eq. (91). For $a$ $\geq 0$, the only homogeneous steady solution is $\langle\phi(x)\rangle=0$ and any initial condition will evolve to this steady state. If $a<0$, the model may develop a front solution of the stable phase $\phi= \pm \sqrt{-a}$ as it invades the unstable phase $\phi=0$. We restrict ourselves to the case $a \geq 0$. The approach of Sec. II.B.3 allows us to describe the effect of the multiplicative noise by means of the effective equation (Santos and Sancho, 1999)

$$
\frac{\partial \phi}{\partial t}=\phi\left(c(0)-a-\phi^{2}\right)+\mathcal{D} \frac{\partial^{2} \phi}{\partial x^{2}} .
$$

The analysis of this equation shows that for noise intensities $c(0)>a$ the former homogeneous solution $\phi=0$ is not stable anymore, and any initial perturbation will grow away from it until nonlinear terms saturate the field. This type of instability produces a front of the more stable phase $\phi_{\mathrm{st}} \sim \sqrt{c(0)-a}$ invading the unstable one, as shown in Fig. 17.

One can evaluate the front velocity by defining the position of the leading part of the front as the value of $z$ for which $\phi(z, t)$ is a small fraction of $\phi_{\mathrm{st}}$. Applying a linear marginal stability analysis (van Saarloos, 1989) to Eq. (104) leads to the following expression for the front velocity:

$$
v=2 \sqrt{c(0)-a} .
$$

This result compares satisfactorily with numerical simulations, as shown in Fig. 18. 


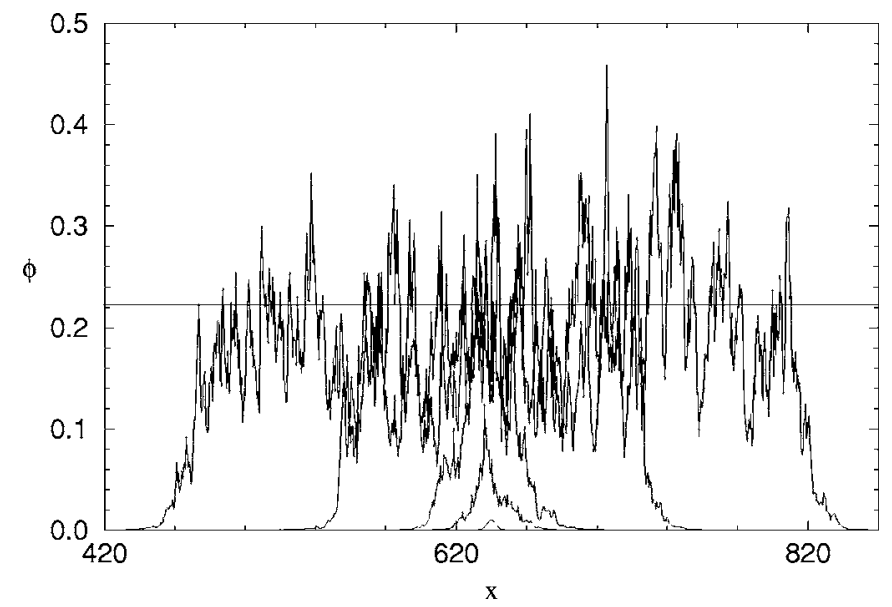

FIG. 17. Four snapshots of an evolving front induced by multiplicative noise in model (91). Times taken at $t=50,100,240$, and 450 from a very small initial perturbation. The continuous line shows the predicted value $\phi_{\mathrm{st}}$. Parameter values: $a=0.1$ and $c(0)=0.15$. From Santos and Sancho, 1999 .

\section{Scaling regime}

We have seen that the EDPT model can reach, in the presence of sufficiently large external fluctuations, a stationary ordered state described by a nonzero order parameter $\langle\phi\rangle$, for both the Itô and Stratonovich interpretations. This means that, if the system is initially in a disordered steady state $\langle\phi\rangle=0$ at a small noise intensity, as the intensity of external fluctuations is increased above its critical value the system develops domains of the two new (symmetric) stationary ordered phases, which grow with time as depicted in Fig. 19. This figure shows that the system behaves differently in the two sto-

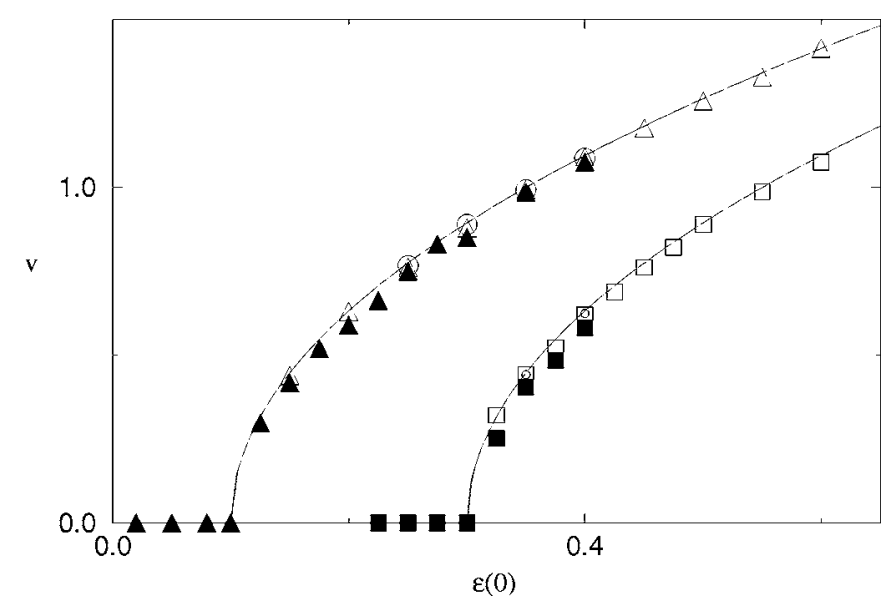

FIG. 18. Mean velocity of the front of Fig. 17 versus noise intensity [denoted here as $\varepsilon(0)$ corresponding to $c(0)$ in the text] for two values of the control parameter, $a=0.1$ (triangles) and $a=0.3$ (squares). Symbols are simulation results and continuous lines are the theoretical predictions given by Eq. (105). Full symbols correspond to simulation parameters $\Delta x=0.5$ and $\Delta t=0.01$, and open symbols for $\Delta x=0.1$ and $\Delta t=0.001$. Circles correspond to the simulation of the effective deterministic model (104). From Santos and Sancho, 1999. chastic interpretations for the same noise intensity, the domains being much more contrasted in the Itô case due to the fact that the order parameter is larger than in the Stratonovich case, which is thus very noisy.

In the next paragraphs, we are concerned with the growth of these noise-induced domains. Irrespective of the originating mechanism, we can expect that once the domains have appeared their dynamics has the same characteristics as that following the quench of a system below its order-disorder transition temperature, as happens in the Ginzburg-Landau (GL) model (Gunton et al., 1983). In the form used here, referred to in the literature as the nonconserved order parameter model, and corresponding, for instance, to phase separation in ferromagnetic systems, one of the domains grows until it fills the whole system. The mechanism underlying domain growth corresponds to the interface motion between domains caused by the interface structure. The translational velocity of the domain boundary has been found to be proportional to its mean curvature, and independent of the free energy of the interface. This can be quantified by the equation of motion obeyed by the characteristic length (i.e., the average radius) of the domains of equilibrium phases $R(t)$ (Gunton et al., 1983),

$$
\frac{d R}{d t}=A \frac{\Gamma}{R}
$$

where $A$ is a model-dependent constant and $\Gamma$ is the kinetic coefficient multiplying the diffusion term. Equation (106) leads in a straightforward way to the AllenCahn law of domain growth $R(t) \propto \sqrt{2 A \Gamma} t^{1 / 2}$. In the time regime where this law is verified, $R(t)$ is the only characteristic length of the system, and scaling behavior for its spatial structure at different times is found. The top plot of Fig. 20 shows the temporal evolution of $R(t)$ for the two stochastic interpretations of the EDPT model, at equal values of the noise intensity. The results show that the Allen-Cahn law is satisfied for both interpretations, and that there is a time regime in which the system is self-similar. One interesting fact is that domain evolution in Itô is slower than in Stratonovich, and in both cases much slower than the Ginzburg-Landau model. This feature can be explained looking at the constant prefactor $\sqrt{2 A \Gamma}$ of the Allen-Cahn law. In the GinzburgLandau model $\Gamma=1$, but in the EDPT model this quantity is field dependent according to Eq. (97), and can be approximated by

$$
\Gamma \approx \frac{1}{1+c\left\langle\phi^{2}\right\rangle} \approx \frac{1}{1+c\langle\phi\rangle^{2}} .
$$

According to Eq. (107), and since for fixed $\sigma^{2}$ we have that $\left.\langle\phi\rangle_{I}\right\rangle\langle\phi\rangle_{S}$, we should expect the slowest growth for the Itô EDPT case, and the fastest one for the GinzburgLandau model, in agreement with the top plot of Fig. 20.

In order to characterize the dynamical scaling of the system, one can let the system evolve from an initial disordered state, and compute the isotropic correlation function $G(r, t)$ at different times. The domain size $R(t)$ is given by the distance at which $G(r, t)$ is half its maxi- 


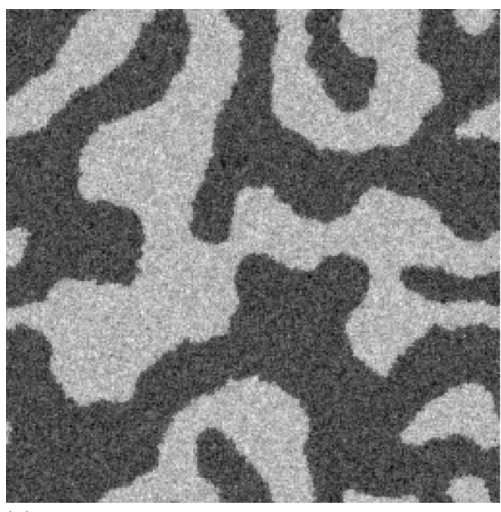

(a)

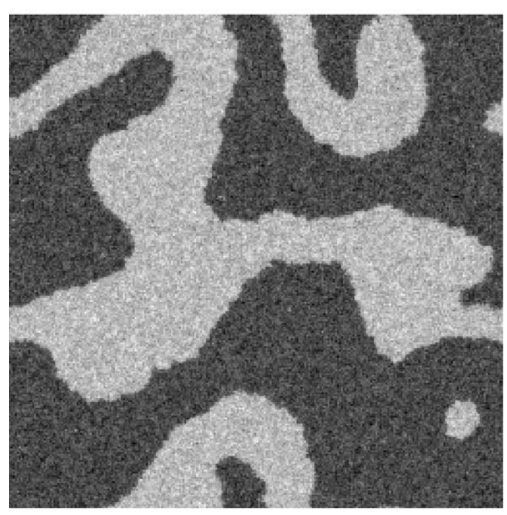

(b)

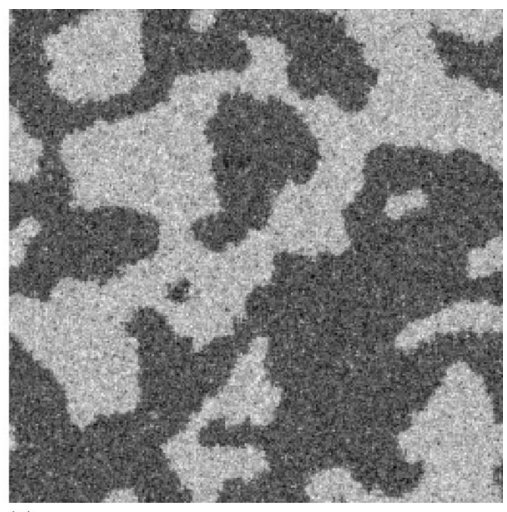

(c)

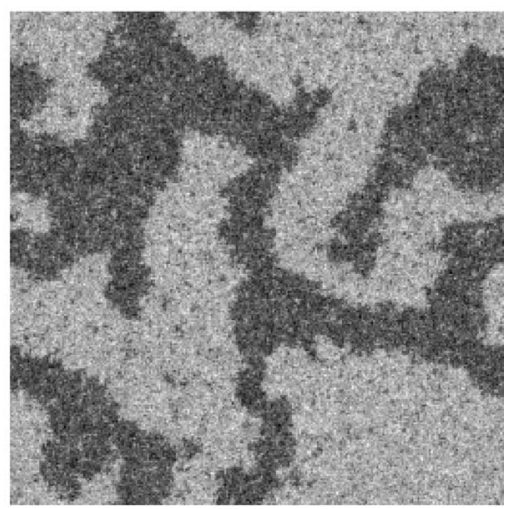

(d)
FIG. 19. Snapshots of evolving noise-induced domains for the EDPT model at $t=750$ (left figures) and $t=1750$ (right figures) in the Itô (top) and Stratonovich (bottom) interpretations. Parameter values: $a=1, c=3, \sigma^{2}=3.5$, and $L=256$. From Carrillo et al., 2003. mum value. Defining $g(r, t) \equiv G(r, t) / G(0, t)$, one can apply the scaling hypothesis for a $d$-dimensional system $g(r, t)=g(r / R(t))$ with no other explicit time dependence. When this relation holds, the spatial structure of the system at different times is statistically equivalent, except for a scale factor. This relation is verified for both interpretations in Fig. 20. Moreover, the scaling function is also the same for both interpretations (bottom plot of Fig. 20), although the evolution of $R(t)$ (top plot) is not.

Noise-induced phase transitions and growth phenomena can also be studied for conserved order parameter models, employed, for instance, in phase separation of binary mixtures, provided the analytical approaches and numerical algorithms are modified adequately. An example has been given by Ibañes et al. (2000).

\section{Spatiotemporally structured noises}

The phase-transition phenomena reviewed so far in this section have mostly involved spatiotemporal white noises. However, there are many experimental situations in which the correlation time and length of the fluctuations are comparable to, or even greater than, the corresponding characteristic time and length scales of the deterministic system. Several studies have addressed the influence of the spatiotemporal correlation of the noise on nonequilibrium phase transitions. In particular, the noise correlation time and length can be used as control parameters of the phase transition, usually with a disordering role. For example, the correlation time and length of an additive spatiotemporal noise control a phase transition toward disorder in the GinzburgLandau model (3), without changing the universality class from that of equilibrium (García-Ojalvo and Sancho, 1994). The role of a spatially correlated multiplicative noise was studied in model (91) by Ibañes et al. (1999).

The theoretical approach to non-Markovian effects resulting from a temporal correlation of the noise terms is quite complicated (García-Ojalvo and Sancho, 1999); Santos and Sancho, 2001). Mangioni et al. (1997, 2000) developed an effective Markovian approximation to study the model (94) with a noise white in space and colored in time. Their results showed that this type of noise has a disordering effect of the noise-induced phase transition scenario. Interestingly, they showed also that in the presence of temporal correlation disorder is favored for large enough spatial coupling, another counterintuitive effect. Kim et al. (1998), on the other hand, showed that a dichotomous multiplicative noise, acting also on model (94), rendered the disordering reentrant transition discontinuous, giving rise to bistability at large noise levels between the ordered and disordered phases.

\section{STATIONARY PATTERNS}

As reviewed in the Introduction (see Sec. I.B.3), many macroscopic systems undergo transitions toward stationary inhomogeneous patterns provided the system is externally maintained far from equilibrium (Manneville, 1990; Cross and Hohenberg, 1993; Walgraef, 1997). Para- 

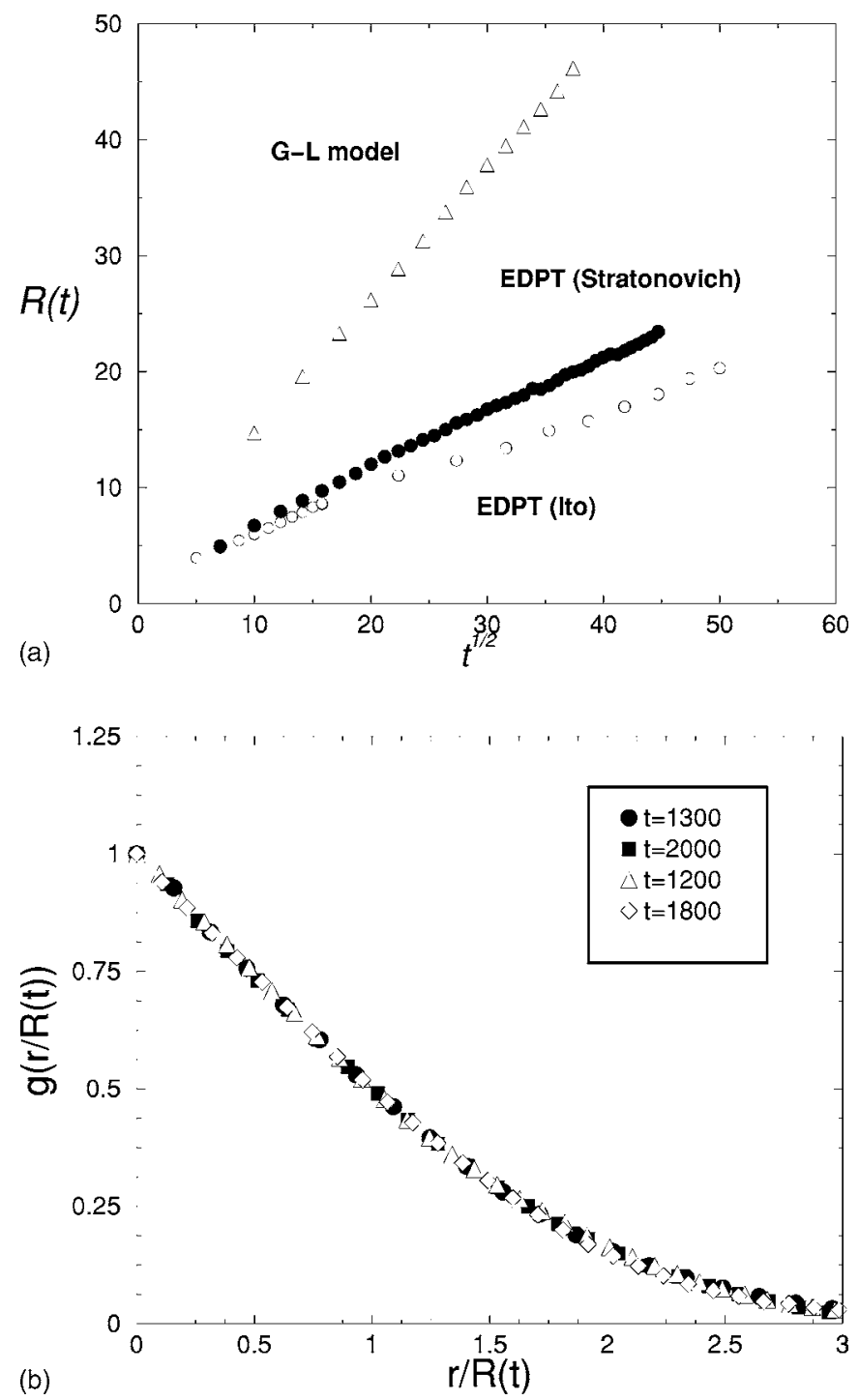

FIG. 20. Scaling of noise-induced domain growth. Top: AllenCahn law for the GL and EDPT models for equal noise intensities (and different mean fields). The latter is computed in both the Itô and Stratonovich interpretations. Bottom: Scaled pair correlation function for the EDPT model in the Stratonovich interpretation for $t=1300$ (circles) and $t=2000$ (squares), and in the Itô interpretation for $t=1200$ (triangles) and $t=1800$ (diamonds). Parameter values: $a=1, c=3, \mathcal{D}=4$, and $\sigma^{2}=3.5$. From Carrillo et al., 2003 .

digmatic examples already mentioned are convective structures arising in Rayleigh-Bénard cells (Chandrasekhar, 1981) and Turing patterns displayed by certain chemical reactions (Castets et al., 1990). We review in this section the influence of spatiotemporal noise on these pattern-forming instabilities. In particular, we describe two different mechanisms through which noise induces patterns in these types of models. We also review an experimental observation of Turing patterns induced by spatial noise. These results may be relevant to realistic situations in which noise is found to induce spatial steady patterns in natural systems such as ecosystems (Satake et al., 1998; Spagnolo et al., 2003).

\section{A. Noisy precursors near pattern-forming bifurcations}

Stationary patterns usually arise via the instability of a homogeneous state versus perturbations with zero frequency and nonzero spatial wave number. The canonical model of such a pattern-forming bifurcation is the SwiftHohenberg equation (4). As explained in Sec. I.B.3, this equation exhibits a stationary bifurcation of the trivial homogeneous solution $\phi=0$ at a critical nonzero wave number. This pattern-forming bifurcation can be considered the spatial analog of the Hopf bifurcation, in which a steady state becomes unstable versus perturbations with zero spatial wave number and nonzero frequency. It is well known, see Sec. I.D.3, that close to (but below) a Hopf bifurcation noise is able to advance the temporal periodicity that arises beyond threshold (Wiesenfeld, 1985). Corresponding "spatial" noisy precursors also exist for stationary pattern-forming bifurcations. This can be readily seen by studying the behavior of the statistical moments of the field $\phi(\mathbf{x}, t)$ in the presence of the additive noise term $\xi(\mathbf{x}, t)$. Below threshold $(a<0)$, and assuming a small noise intensity, the nonlinear term in Eq. (4) can be neglected. In Fourier space, and for noise with zero mean, the equation for the first moment reads

$$
\frac{\partial\langle\hat{\phi}\rangle}{\partial t}=a\langle\hat{\phi}\rangle-\left(k^{2}-k_{0}^{2}\right)^{2}\langle\hat{\phi}\rangle,
$$

where $\hat{\phi}(\mathbf{k}, t)$ is the Fourier transform of $\phi(\mathbf{x}, t)$. The stationary solution of this equation is $\hat{\phi}_{\mathrm{st}}(\mathbf{k}, t)=0$. On the other hand, the evolution equation for the structure function $S(\mathbf{k}, t)=\langle\hat{\phi}(\mathbf{k}, t) \hat{\phi}(-\mathbf{k}, t)\rangle$ can be obtained by writing the linear version of Eq. (4) in the lattice, deriving from it the equation for the discrete version of $S(\mathbf{k}, t)$, and transforming the resulting equation back to continuum Fourier space. The final result is (GarcíaOjalvo and Sancho, 1999)

$$
\frac{\partial S(\mathbf{k}, t)}{\partial t}=2\left[a-\left(k^{2}-k_{0}^{2}\right)^{2}\right] S(\mathbf{k}, t)+2 \sigma^{2} .
$$

The steady state of this equation is easily evaluated,

$$
S_{\mathrm{st}}(k)=\frac{\sigma^{2}}{\left(k^{2}-k_{0}^{2}\right)^{2}-a},
$$

which has a maximum at the critical wave number $k_{0}$. This quantity is plotted as a function of wave number $k$ in Fig. 21. This result shows that, even below threshold, random fluctuations extract the intrinsic spatial scale that arises at the pattern-forming bifurcation, analogously to the noisy precursors found near Hopf bifurcations.

Such spatial noisy precursors have been observed experimentally in liquid crystals (Rehberg et al., 1991; Qiu and Ahlers, 2005), nonlinear optical media (Agez et al., 2002), Rayleigh-Bénard convection (Wu et al., 1995), and vibrated granular media (Goldman et al., 2004). Figure 22 shows the structure function of the spatial distribution of density fluctuations in a vertically vibrated granular layer, as reported by Goldman et al. (2004), both be- 


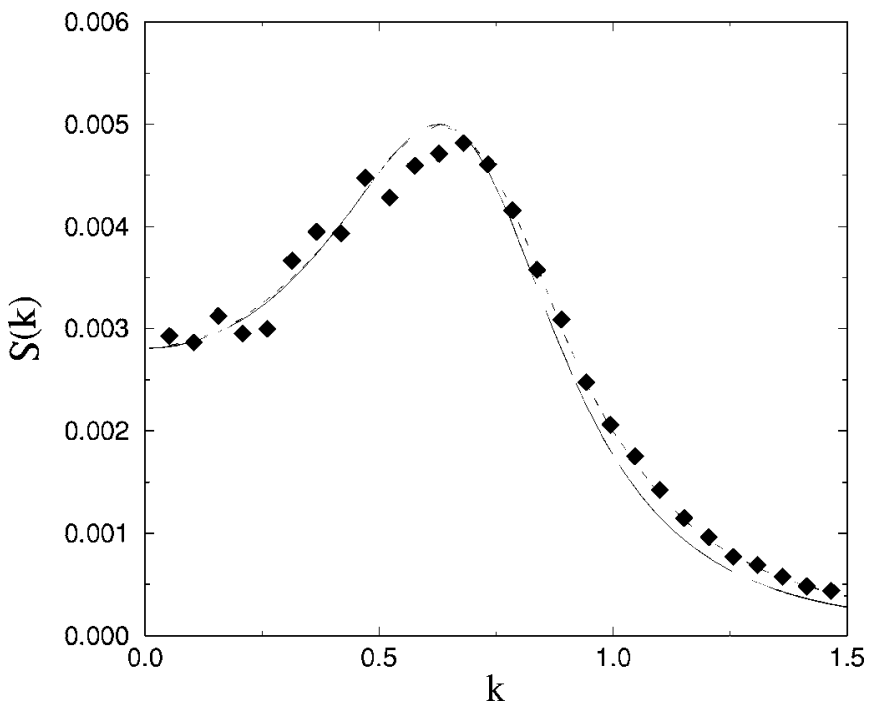

FIG. 21. Stationary structure function of the Swift-Hohenberg equation (4) with additive noise $(d=2)$. Symbols represent numerical simulations of the equation below threshold including the nonlinear term, the solid line is the linear solution (110), and the dashed line corresponds to the numerical integration of the discrete evolution equation. Parameter values: $a=-0.2$, $\sigma^{2}=0.001$, and $k_{0}=0.63$. From García-Ojalvo and Sancho, 1996 .

low and above the pattern-forming transition. It can be seen that below the corresponding pattern-forming threshold a circular-ring-shaped structure function indicates that fluctuations are exciting patterns with a welldefined wavelength but with no well-defined direction. Above the bifurcation, the structure function ring collapses into a finite number of peaks, chosen by the system via some kind of pattern-selection mechanism (Ciliberto et al., 1988; Ramazza et al., 1996). In Sec. V.B.3, we will make use of this result to discuss the phenomenon of spatial stochastic coherence.

\section{B. Noise-induced patterns via a short-time instability}

Analogously to noise-induced phase transitions (see Sec. III), multiplicative noise is able to induce stationary patterns in extended systems as well. Also here, the first mechanism that was shown to lead to noise-induced patterns was the instability of a homogeneous state, as described in what follows.

Consider the Swift-Hohenberg model (4) in which the control parameter $a$ fluctuates in space and time, i.e., $a$ $\rightarrow a+\eta(\mathbf{x}, t)$, where $\eta(\mathbf{x}, t)$ is a Gaussian noise with zero mean and correlation given by the $d$-dimensional version of Eq. (60). The influence of the multiplicative noise on the pattern-forming bifurcation can be determined by a linear stability analysis of the homogeneous solution $\phi=0$ (Becker and Kramer, 1994). This analysis can be performed, for instance, on the linearized equation of the structure function, which in the presence of multiplicative noise and, following the procedure described in the context of Eq. (109) above (García-Ojalvo and Sancho, 1999), takes the form

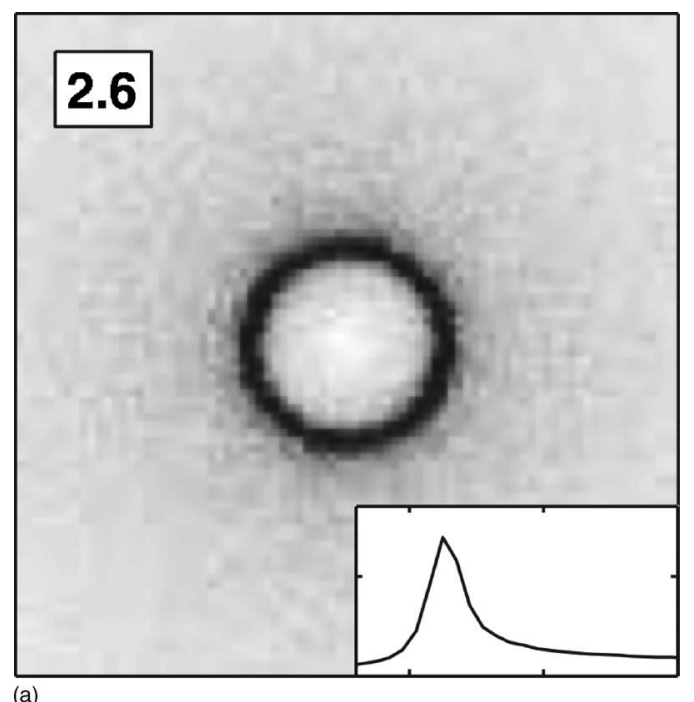

(a)

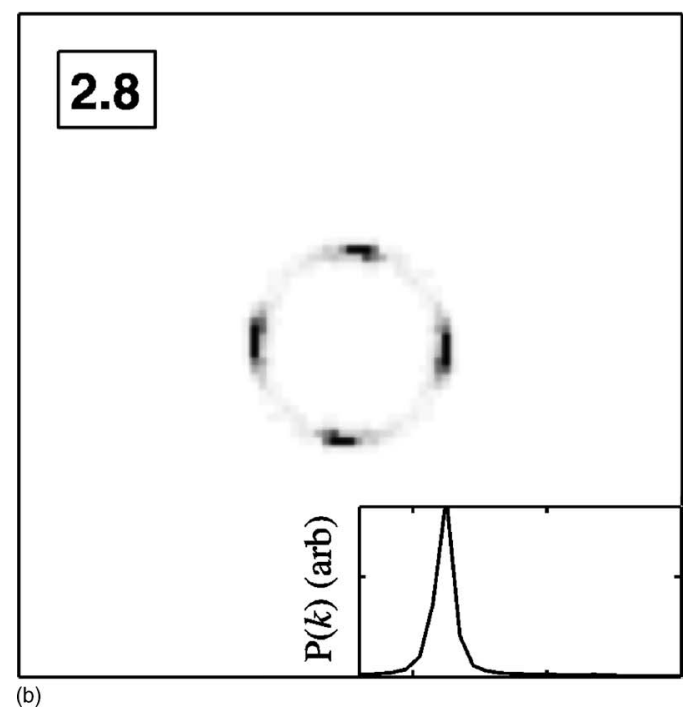

FIG. 22. Spatial noisy precursor in an oscillated granular layer. Experimentally determined structure function (top) below and (bottom) above the onset of standing waves. In each plot, the top left inset gives the numerical value of the peak acceleration of the plate relative to gravity, and the bottom right inset depicts the circularly averaged structure function that can be compared with that in Fig. 21. From Goldman et al., 2004.

$$
\begin{aligned}
\frac{\partial}{\partial t} S(\mathbf{k}, t)= & 2\left[a+c(0)-\left(k^{2}-k_{0}^{2}\right)^{2}\right] S(\mathbf{k}, t)+2 \sigma^{2} \\
& +2 \frac{1}{(2 \pi)^{2}} \int \hat{c}(\mathbf{q}) S(\mathbf{k}-\mathbf{q}, t) d \mathbf{q} .
\end{aligned}
$$

The last term in this equation can be ignored close enough to the bifurcation point, since it is of higher order on noise intensity. As a consequence, the stability of the homogeneous solution, which in the absence of additive noise is given by $S(\mathbf{k}, t)=0$, is now controlled by the first term on the right-hand side (r.h.s.) of Eq. (111). Clearly, the existence of a multiplicative noise of intensity given by $c(0)$ produces a shift in the critical point, in such a way that now its value in a first-order approximation is $a_{c}=-c(0)$. In other words, patterns can exist for a 
(a)

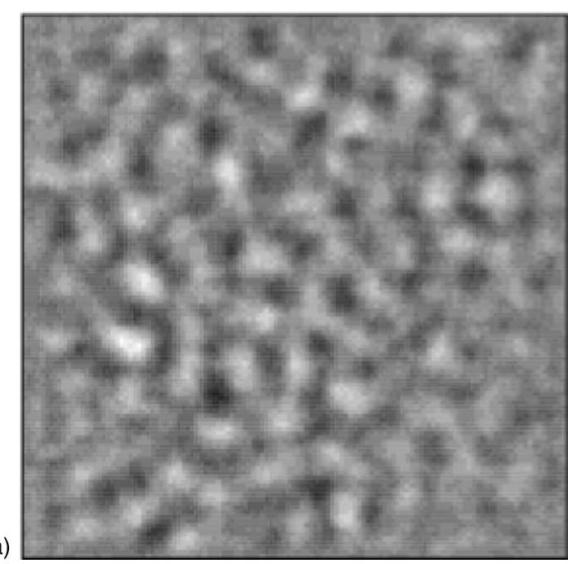

(b)

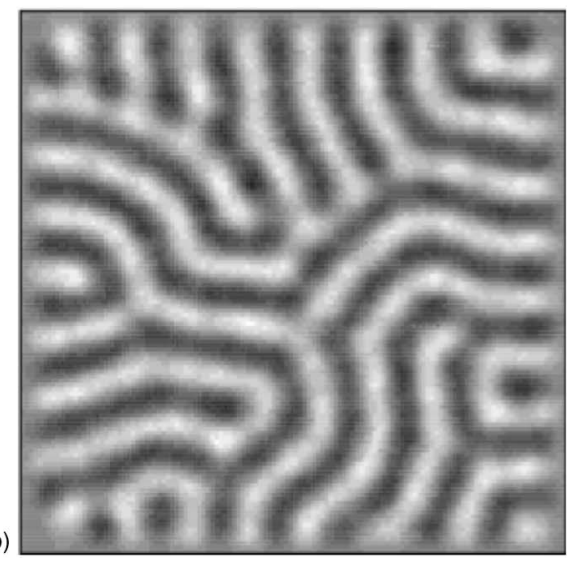

FIG. 23. Patterns exhibited by the Swift-Hohenberg equation (4) for a deterministically subthreshold value of $a$ in the (top) absence and (bottom) presence of a multiplicative noise of sufficient intensity. The bottom plot (no multiplicative noise) displays the characteristic spatial noisy precursors described in Sec. IV.A. Multiplicative noise (top plot), on the other hand, destabilizes the previous state and leads to wave vectors with locally well-defined directions, corresponding to a roll pattern. Parameter values: $a=-0.05, \sigma^{2}=0$ (top), and $\sigma^{2}=0.1$ (bottom). The intensity of internal additive noise is $10^{-3}$ in both cases. From García-Ojalvo et al., 1993.

deterministically subthreshold value of the control parameter $a$. An example from the first theoretical prediction of this phenomenon (García-Ojalvo et al., 1993) is shown in Fig. 23.

One way to characterize this transition is by defining the convective flux $J$ as

$$
J(t) \equiv \frac{1}{V} \int \phi^{2}(\mathbf{r}, t) d \mathbf{r},
$$

which will act as the order parameter in this patternforming scenario. Below the threshold, we expect that in the conducting state $J_{\text {st }} \sim 0$ but $J_{\text {st }}>0$ in the convective or patterned state. This behavior can be seen in Fig. 24, where the transition between these two states is controlled by the intensity of the multiplicative noise.

Following this result, the same approach was used by Parrondo et al. (1996) to study patterns for a noiseinduced phase transition [see Eq. (94)]. These authors

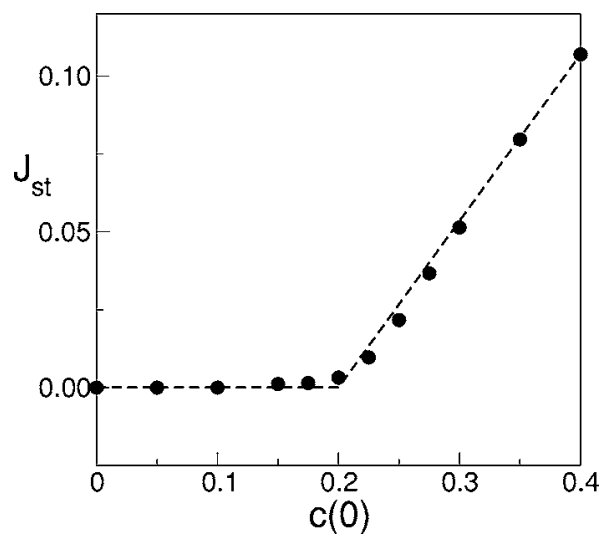

FIG. 24. Convective steady flux $J_{\text {st }}$ versus multiplicative noise intensity $c(0)$. Parameter values: $a=-0.2$ and $k_{0}=0.63$. Adapted from García-Ojalvo and Sancho, 1994.

introduced a Swift-Hohenberg-like spatial coupling, instead of the standard diffusion operator, to induce a well-defined spatial periodicity in the ordered phase. A convenient generalization of the mean-field approach described in Sec. II.B.1 was able to predict qualitatively the appearance of these noise-induced patterns. More details of this adapted MFA will be given in the next section.

Additionally, Zaikin and Schimansky-Geier (1998) showed that patterns can also be induced by additive noise, provided multiplicative noise is also present in the system. A theoretical approach based on the study of higher-order moments (see Sec. II.B.4), introduced by Dutta et al. (2005), can be used to describe analytically the effect of additive noise on these noise-induced phenomena.

From an applied perspective, noise-induced patterns have been studied in theoretical models of electrohydrodynamic convection in liquid crystals (Behn et al., 1998), semiconductor nanostructures (Stegemann et al., 2005), and adsorption in surfaces (Mangioni and Wio, 2005).

\section{Entropy-driven patterns}

Even in the absence of short-time instabilities, noise can induce patterns in nonlinear media. This is again analogous to the case of phase transitions, where noise may induce changes in the steady-state local probability distribution. In the presence of an adequate spatial operator, a noise-induced pattern arises (Buceta et al., 2003). The role of noise here is not to produce an instability, but to excite the system away from the homogeneous state, a tendency that is balanced by the relaxing character of the deterministic force. This mechanism is reviewed in detail in the following paragraphs.

Consider the model described in Eq. (96), but with spatial coupling of the Swift-Hohenberg type,

$$
\frac{\partial \phi}{\partial t}=\Gamma(\phi)\left[-a \phi-\mathcal{D}\left(\nabla^{2}+k_{0}^{2}\right)^{2} \phi\right]+\Gamma(\phi)^{1 / 2} \eta(\mathbf{x}, t),
$$

where the kinetic coefficient $\Gamma(\phi)$ is given by Eq. (97) and the multiplicative noise is white in space and time 
with correlation given by the $d$-dimensional version of Eq. (29). In the absence of noise, this system has a fixed point $\phi=0$, whose stability in the presence of noise can be established by a linear stability analysis of Eq. (113). First, we write the field in terms of its spatial Fourier modes,

$$
\phi(\mathbf{x}, t)=\sum_{k} \hat{\phi}_{k}(t) e^{i \mathbf{k} \cdot \mathbf{x}} .
$$

Linearizing Eq. (113) around $\phi=0$, using Eq. (114), and integrating leads to

$$
\begin{aligned}
\phi(\mathbf{x}, t) & =\sum_{k} \hat{\phi}_{k} \exp [\omega(\mathbf{k}) t] \exp (i \mathbf{k} \cdot \mathbf{x}) \\
& =\sum_{k} \hat{\phi}_{k} \exp \left\{\left[-a-\sigma^{2} c-\mathcal{D}\left(k_{0}^{2}-k^{2}\right)^{2}\right] t+i \mathbf{k} \cdot \mathbf{x}\right\} .
\end{aligned}
$$

Since $a, c$, and $\mathcal{D}$ are positive, this result clearly shows that the disordered phase $\phi=0$ is not affected by a linear instability at short times. Although all wave vectors relax to zero, a particular one with $\mathbf{k}^{*}$ is such that $\omega\left(\mathbf{k}^{*}\right)$ $=0$, which is close to $k_{0}$ (and equal to it in the continuum limit).

Interestingly enough, however, this model exhibits noise-induced patterns via an entropic mechanism. To prove that, we use a generalized version of the meanfield approximation, valid for the case in which the ordered phase exhibits a pattern. As discussed in Sec. II.B.1, we expect that the spatial coupling term in Eq. (113) will lead to an interaction between the local field and neighboring values that we assume to lead to the effective SDE,

$$
\frac{d \phi}{d t}=\Gamma(\phi)\left\{-a \phi-\mathcal{D}_{1}(\phi-\langle\phi\rangle)\right\}+\Gamma(\phi)^{1 / 2} \eta(t) .
$$

This can be achieved with the ansatz

$$
\phi_{\mathbf{x}^{\prime}}=\langle\phi\rangle \cos \left[\mathbf{k}^{*} \cdot\left(\mathbf{x}-\mathbf{x}^{\prime}\right)\right] .
$$

Actually, under this assumption we transform Eq. (113) into Eq. (116), in terms of an effective diffusion parameter,

$$
\mathcal{D}_{1}=\mathcal{D}\left[\left(\frac{2 d}{(\Delta x)^{2}}-k_{0}^{2}\right)^{2}+\frac{2 d}{(\Delta x)^{4}}\right] .
$$

The Fokker-Planck equation associated with Eq. (116) can be solved in the steady state in a straightforward way. The resulting stationary probability distribution reads

$$
\begin{aligned}
P(\phi ;\langle\phi\rangle)= & \mathcal{N}\left(1+c \phi^{2}\right)^{1 / 2} \\
& \times \exp \left\{-\frac{1}{\sigma^{2}}\left[\frac{a+\mathcal{D}_{1}}{2} \phi^{2}-\mathcal{D}_{1}\langle\phi\rangle \phi\right]\right\},
\end{aligned}
$$

where $\mathcal{N}$ is a normalization constant. The usual selfconsistent condition must hold,

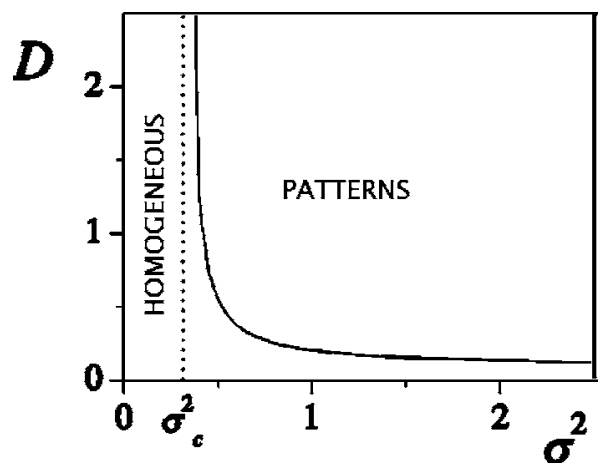

FIG. 25. Phase diagram showing the boundary line delimiting the region of existence of a patterned state (defined by $\langle\phi\rangle$ $\neq 0$ ) produced by an entropic mechanism. The boundary line was estimated with a generalized mean-field approach (see text). The vertical dashed line indicates the location of the noise-induced transition of the local potential, see Eq. (99). Parameter values: $k_{0}=1, a=1$, and $c=3$. Adapted from Buceta et al., 2003.

$$
\langle\phi\rangle=\int_{-\infty}^{\infty} \phi P(\phi ;\langle\phi\rangle) d \phi
$$

This equation has always the solution $\langle\phi\rangle=0$, since in that case the probability distribution (119) is symmetric. A nonzero solution is also possible if we increase $\sigma^{2}$. In Fig. 25, the domains of homogeneous and spatially patterned solutions are plotted, and it shows that model (113) may exhibit stationary patterns for large enough coupling parameter $\mathcal{D}$ and noise intensity $\sigma^{2}$. Examples of the resulting patterns are shown in Fig. 26. This figure presents snapshots of the spatiotemporal behavior of model (113) for increasing noise (from A to C).

\section{Turing patterns}

As mentioned in Sec. I.B.3, Turing patterns appear as a consequence of stationary pattern-forming bifurcations in two-component systems with activator-inhibitor dynamics, provided the relaxation length of the inhibitor is sufficiently larger than that of the activator. The first experimental evidence of Turing instabilities was reported for the chlorite-iodide-malonic acid (CIMA) re-

$$
\phi=-6 \quad \phi=+6
$$
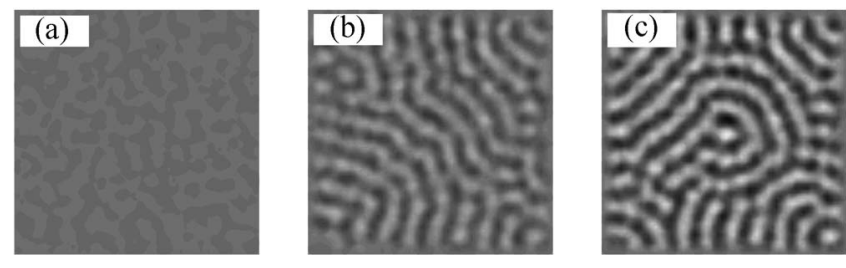

FIG. 26. Patterns induced by noise via an entropic mechanism associated to the Swift-Hohenberg spatial operator [see Eq. (113)]. Parameter values are those of Fig. 25, plus $\mathcal{D}=5$ and (a) $\sigma^{2}=0.1$, (b) 2, and (c) 5. Adapted from Buceta et al., 2003. 

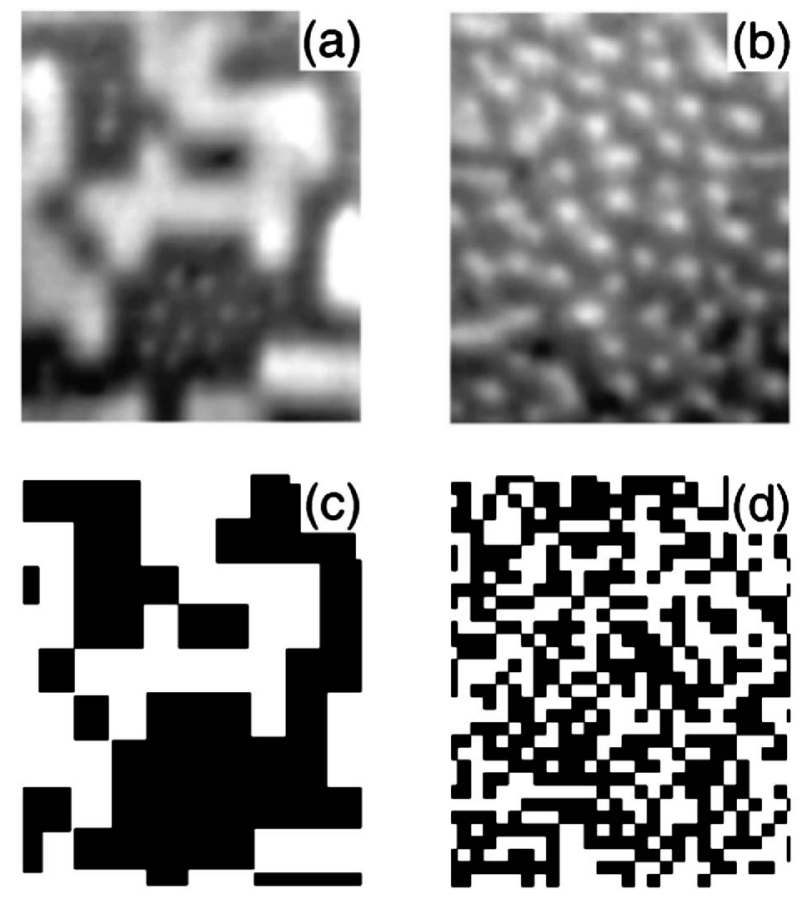

FIG. 27. Turing pattern induced by spatial noise in the CDIMA reaction. (a),(b) Experimental snapshots under the illumination profiles shown in (c) and (d), respectively. Black and white in (c) and (d) denote two different illumination levels, low and high, respectively. From Sanz-Anchelergues et al., 2001.

action (Castets et al., 1990). Later on, an extension of the reaction was proposed, the so-called CDIMA reaction, which has the important feature of being photosensitive (Muñuzuri et al., 1999) (see Sec. I.B.3). This has made it possible to analyze the influence of external noise in Turing pattern bifurcations. To study that influence, Sanz-Anchelergues et al. (2001) used a static illumination profile, consisting of a random pattern of two different illumination levels, acting upon a CDIMA reactor. Experimental snapshots of the system behavior under these conditions are shown in Fig. 27.

Figure 27 shows optical images of the chemical reactor (top row) for two different illumination conditions (bottom row). The case shown in the left column corresponds to a long-wavelength illumination profile [plot (c)], where it can be assumed that the black and white regions are independent of each other. The resulting behavior [plot (a)] clearly shows that Turing spots appear in the regions of low illumination, whereas the system is homogeneous for high illumination. For a random illumination profile with shorter wavelength [plot (d)], on the other hand, the spatial scale of the noise interacts with the internal spatial scale of the system, whose spatial behavior no longer mimics the illumination profile. Instead, a well-defined Turing pattern appears throughout the system [plot (b)], even though the average illumination is such that a homogeneous illumination at the same level would produce no pattern.

Sanz-Anchelergues et al. (2001) reproduced the experimental behavior with the Epstein-Lengyel activator-

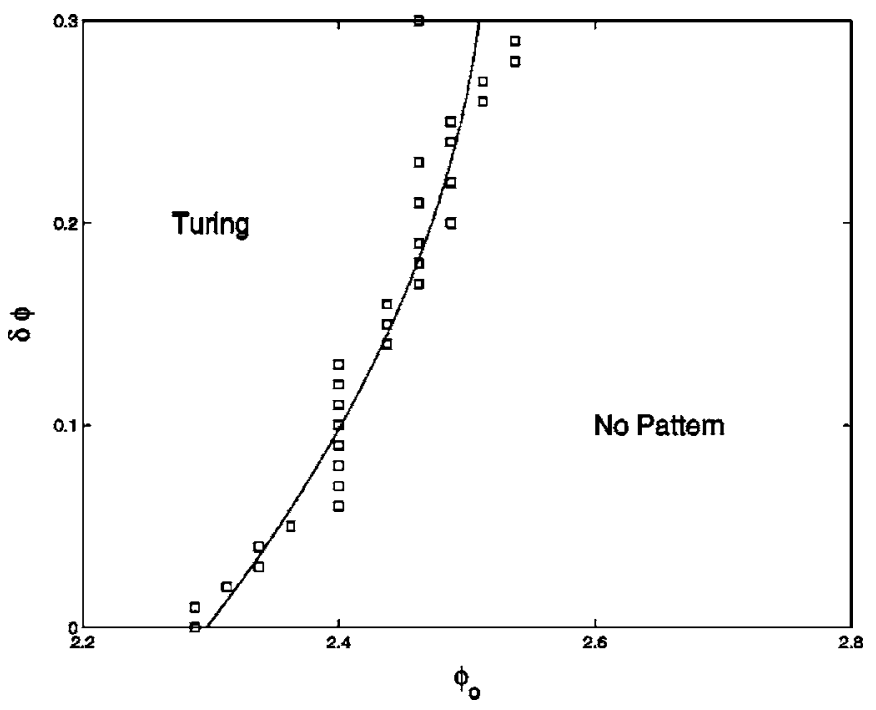

FIG. 28. Numerical phase diagram of the Turing instability of the CDIMA reaction for varying levels of average illumination and noise. Parameter values: $a=16, c=0.6, \sigma=301$, and $d$ =1.07. From Sanz-Anchelergues et al., 2001 .

inhibitor model adapted to the photosensitive version of the CDIMA reaction [Eq. (5)]. According to the experiment, the spatially patterned illumination consists in a symmetric dichotomous spatial noise of amplitude $2 \delta \phi$, around a mean value $\phi_{0}$. The numerically computed behavior of the system for various levels of noise and mean illumination is shown in Fig. 28. It is evident that as the amplitude of the illumination noise increases, the region where Turing patterns exist increases correspondingly. This is therefore clear evidence that spatial noise induces order in the system. An extension to time-varying noise would be of interest.

\section{SPATIOTEMPORAL DYNAMICS: NOISE-UNVEILED COHERENCES}

A large number of cases in which random fluctuations exert a constructive influence involve situations in which order is already present, albeit hidden, in the system. In those instances, noise is able to unveil this implicit order. This is the case, for example, of bistable systems with external harmonic forcing. These systems have the potential to display coherent (periodic) output under deterministic conditions, provided the forcing amplitude is large enough. In the case of weak forcing, on the other hand, the periodic response is absent without fluctuations, but the noise is able to excite it and amplify. A similar phenomenon arises in systems with well-defined internal time scales (such as excitable media), where no external driving is needed, but noise is still able to unveil a hidden periodicity in the system. In what follows, we review these and other examples of noise-unveiled coherences in extended media, placing the emphasis on the influence of spatial coupling in this type of behavior. 


\section{A. Forced systems}

Among the many constructive roles of noise, one of the clearest (and definitely the best known) examples is stochastic resonance (Wiesenfeld and Moss, 1995). In its simplest form, stochastic resonance (SR) consists in an amplification of the response of a nonlinear system to a weak temporal forcing for an optimal amount of noise. SR has been observed experimentally in a wide variety of situations [see, e.g., Gammaitoni et al. (1998); Anishchenko et al. (1999) for comprehensive reviews]. As we show in the next subsection, stochastic resonance in extended media exhibits distinctive properties. To clarify the notation, we define two additional scenarios that will also be reviewed in the final subsection. What could be considered in a sense the spatial counterpart of stochastic resonance, i.e., the ordering effect of noise in extended systems forced with a spatially periodic signal, is referred to here under the term spatial stochastic resonance. Finally, another option is still possible. We denote it as spatiotemporal stochastic resonance, and describe under this concept resonant effects mediated by noise in extended media forced with spatiotemporal signals, rather than with pure temporal inputs.

\section{Stochastic resonance in extended media}

\section{a. Discrete arrays}

The effect of coupling on the response of stochastic systems to weak signals has been analyzed in studies of globally coupling arrays of bistable elements (Jung et al., 1992; Bulsara and Schmera, 1993) [see also Morillo et al. (1995)]. Those early investigations showed that coupling noticeably enhances the stochastic resonance effect. Numerous studies were also devoted to the detection of weak signals using ensembles of uncoupled summing elements, mostly in a neurophysiological context (Pei et al., 1996; Lindner and Schimansky-Geier, 2001). In particular, it was shown that the traditional SR requirement of operating close to threshold under periodic forcing could be lifted (Collins et al., 1995; Stocks, 2000), which makes the phenomenon more relevant for biological signal transduction (Bezrukov and Vodyanoy, 1995). In the intermediate case between global and no coupling, a number of studies have been devoted to the case of local coupling. The simplest situation was addressed by Neiman and Schimansky-Geier (1995) with a complete theoretical analysis of the two-element case. Experimental related evidence has more recently been provided in a system of only two bidirectionally coupled semiconductor lasers, showing a dramatic improvement in the collective response of the system to external periodic force with respect to the uncoupled case (Buldú et al., 2002, 2005). Referring to extended arrays, Lindner et al. (1995) first analyzed numerically the case of a onedimensional chain of locally coupled bistable overdamped devices with global harmonic driving,

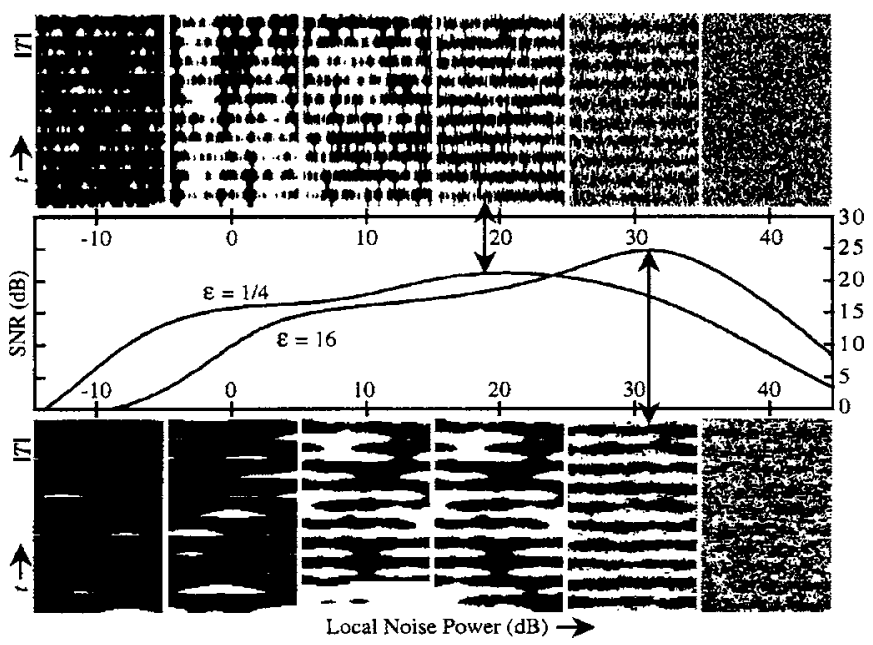

FIG. 29. Array-enhanced stochastic resonance in a onedimensional chain of 101 bistable elements. Spatiotemporal plots, with the oscillator index in the horizontal axis and time advancing upwards, are shown for increasing noise levels (left to right), and two different coupling strengths, with the largest one at the bottom. The corresponding SNR curves are also shown in the middle plot. From Lindner et al., 1995.

$$
\frac{d \phi_{i}}{d t}=a \phi_{i}-\phi_{i}^{3}+\varepsilon \sum_{j \in \operatorname{nn}(i)}\left(\phi_{j}-\phi_{i}\right)+A \cos \omega t+\xi_{i}(t),
$$

where the sum runs over nearest neighbors of element $i$ and the noise $\xi_{i}(t)$ is assumed white in space and time with correlation given by Eq. (31). In the case $\varepsilon=0$, each one of the elements in the chain is a stochastic resonator in which the periodic input is amplified by an optimal amount of noise. The system's response to the driving can be characterized by the signal-to-noise ratio (SNR), defined as the ratio of the output spectral power at frequency $\omega$ to the noise spectral power. The hallmark of stochastic resonance is a maximum of the SNR for a nonzero noise intensity.

In the presence of coupling, $\varepsilon \neq 0$, Lindner et al. (1995) observed that the SNR was higher than in the uncoupled case. Figure 29 shows spatiotemporal plots of the system for increasing noise levels and two different values of the coupling strength $\varepsilon$. The corresponding SNR curves are also shown. The figure reveals the typical signature of stochastic resonance, with the SNR first increasing and later decreasing with noise. The maximum SNR is higher for the largest coupling strength, and occurs at a larger noise level. The spatiotemporal plots show a good global coherence, with basically all elements switching states simultaneously in a periodic way, at the optimal noise level. This phenomenon has been called arrayenhanced stochastic resonance (AESR).

We have seen so far that the maximum SNR increases with coupling strength. However, this increase cannot be monotonous, since in the limit of very high coupling the system should behave as a single oscillator, and therefore its response to the external driving should again be worse than for intermediate coupling values. This is in- 


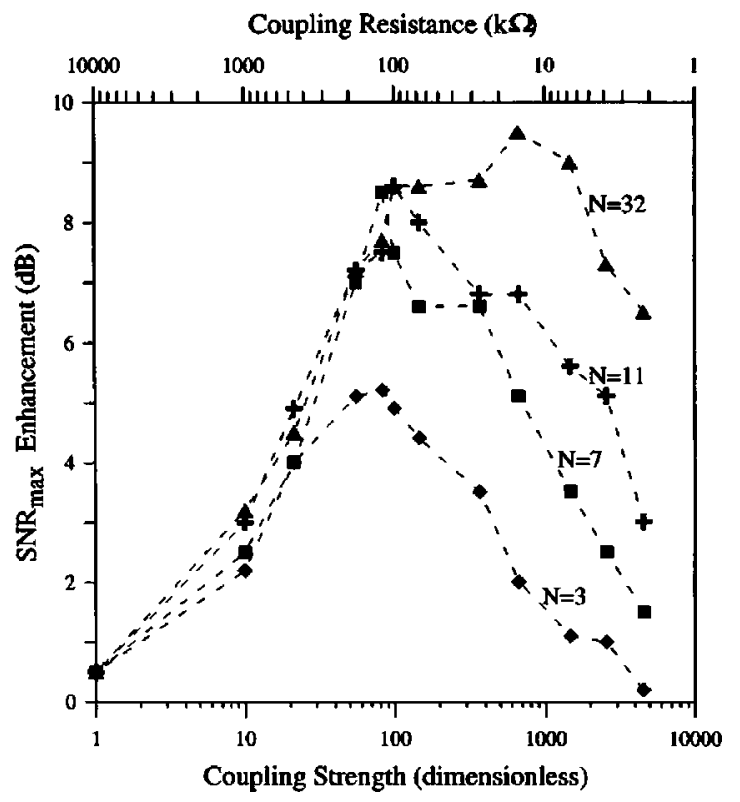

FIG. 30. Experimental results showing the dependence of the maximum SNR on the coupling strength. Measures were made on a linear array of bistable diode resonators, coupled bidirectionally by means of resistors. From Löcher et al., 1996.

deed what happens. Figure 30 shows the dependence of the maximum SNR on the coupling strength for different system sizes, as measured experimentally by Löcher et al. (1996) in a linear array of bistable diode resonators. The figure also shows that this enhancement effect increases for larger system sizes, highlighting even further the constructive influence of the spatial degrees of freedom in this phenomenon. A similar behavior had been previously reported numerically (Lindner et al., 1995, 1996). The phenomenon was also studied experimentally by Rowe and Etchegoin (2001) in an array of bistable electronic triggers.

Subsequent, mostly numerical simulation studies have provided further evidence of the constructive role of coupling in the phenomenon of stochastic resonance. These include the treatment of excitable systems (Chialvo et al., 1997; Tanabe et al., 1999; Zhou, Kurths, and Hu, 2003), coupled map lattices (Gade et al., 1997), Ising systems (Neda et al., 1999), small-world networks of bistable units (Gao et al., 2002), and arrays of monostable elements (Lindner et al., 2001); see also Sec. VI.B.2. Further analysis has been performed by Gang et al. (1996) in arrays of bistable elements composed of both coupling activators and suppressors, Kim et al. (1999) in delayed (globally) coupled arrays of oscillators, and Kanamaru et al. (2001), who applied a mean-field approach to an array of FitzHugh-Nagumo elements. Lai et al. (2004) proposed the use of AESR to enhance signal detectability and suppress jamming noise in oscillator arrays. The relation between aperiodic stochastic resonance and phase synchronization was examined by Park et al. (2004) in an array of excitable units. Volkov, Ullner, et al. (2003) studied the effect of coupled inhibition in arrays of excitable elements, finding a nontrivial dependence of the system's response on the driving frequency, due to the appearance of multiple characteristic frequencies of the coupled system. A recent theoretical treatment of AESR can be found in Lindner et al. (2006).

Noise correlation parameters have been much less analyzed in relation to AESR. Simultaneous to the early published papers on the subject, Inchiosa and Bulsara (1995) considered global noise in globally coupled arrays of bistable dynamic elements. More recently, correlation length effects on the transmission of (site-to-site different) aperiodic subthreshold signals on a Morris-Lecar neural network were studied by Montejo et al. (2005).

The influence of spatial coupling in the SR scenario is further revealed in the effect of the system size. Consider again Eq. (121) with $a=1$. Replacing the spatial coupling term by a mean-field approximation, we obtain

$$
\frac{d \phi_{i}}{d t}=\phi_{i}-\phi_{i}^{3}+\frac{\varepsilon}{N} \sum_{j=1}^{N}\left(\phi_{j}-\phi_{i}\right)+A \cos \omega t+\xi_{i}(t) .
$$

In the Gaussian approximation, we can assume that $\phi_{i}$ $=\Phi+\delta_{i}$, where $\Phi$ is the mean field and $\delta_{i}$ are independent Gaussian random variables with variance $M$. It can be shown (Pikovsky et al., 2002) that these quantities satisfy

$$
\begin{aligned}
& \dot{\Phi}=\Phi-\Phi^{3}-3 M \Phi+A \cos \omega t+\sqrt{\frac{2 \sigma^{2}}{N}} \xi(t), \\
& \frac{1}{2} \dot{M}=M-3 \Phi^{2} M-3 M^{2}-\varepsilon M+\sigma^{2},
\end{aligned}
$$

where $\xi(t)$ is a white noise of unit variance. This system of equations undergoes a pitchfork bifurcation of the $\Phi=0, M>0$ solution at $\varepsilon_{c}=3 \sigma^{2}$. This bifurcation is supercritical for $\sigma^{2}>2 / 3$, above which the system is bistable. Slightly above the bifurcation we can adiabatically eliminate $M$ from Eq. (124) to get

$$
\dot{\Phi}=b \Phi-c \Phi^{3}+A \cos \omega t+\sqrt{\frac{2 \sigma^{2}}{N}} \xi(t),
$$

where $b$ and $c$ are constants that depend on $\varepsilon$ and $\sigma$. Equation (125) corresponds to a noise-driven bistable system, for which stochastic resonance is well known to occur. However, in this case the effective noise level is not only controlled by $\sigma^{2}$, but also by $N$. Hence, the resonant behavior is also associated with $N$ : the response of the system will be optimal for an intermediate system size. Such a system size resonance is shown in Fig. 31. These results were obtained in a globally coupled system, but the same effect was also reported by Pikovsky et al. (2002) in a locally coupled system, specifically in the Ising model. Similar behavior has been reported in ion channel models (Jung and Shuai, 2001; Schmid et al., 2001) and in ensembles of Hodgkin-Huxley neurons (Wang et al., 2005). 


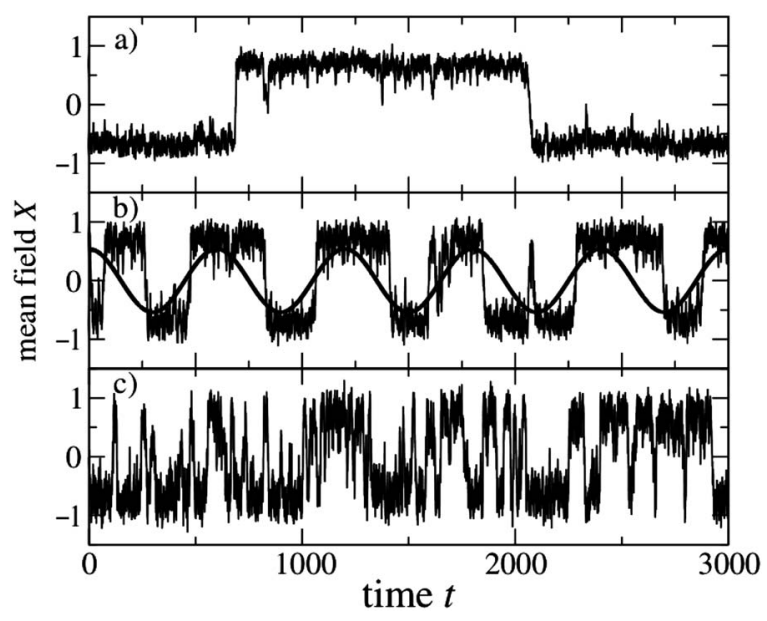

FIG. 31. Time evolution of the mean field of model (122) for three system sizes: (a) $N=80$, (b) $N=35$, and (c) $N=15$. The driving force is superimposed in (b). Parameters values: $\sigma^{2}$ $=0.5, \varepsilon=2, A=0.02$, and $\omega=\pi / 300$. From Pikovsky et al., 2002 .

\section{b. Continuous media}

The concept of stochastic resonance was generalized to continuous extended media soon after Benzi et al. (1981) first reported its existence in stochastic ODEs. Only four years later those same authors studied the influence of a spatiotemporal white noise in the onedimensional Ginzburg-Landau model with global periodic forcing (Benzi et al., 1985),

$$
\frac{\partial \phi}{\partial t}=a \phi-\phi^{3}+\mathcal{D} \frac{\partial^{2} \phi}{\partial x^{2}}+F(t)+\xi(\mathbf{x}, t),
$$

where $a>0$ and $\xi(\mathbf{x}, t)$ represents a white noise in both space and time, with correlation (29). $F(t)$ stands for a uniform dichotomous time-periodic function ${ }^{4}$ that jumps between the values $+B$ and $-B$ with frequency $\omega$. In the absence of forcing, Eq. (126) has two stable uniform steady states $\phi_{ \pm}= \pm \sqrt{a}$ and an unstable one at $\phi_{0}=0$. Assuming Neumann boundary conditions $\partial_{x} \phi(0, t)$ $=\partial_{x} \phi(L, t)=0$, where $L$ is the system size, a set of spacedependent unstable steady states $\phi^{(k)}(x)$ exist, defined by the number $k$ of crossings of the $\phi=0$ axis.

In the presence of forcing, each of the two subcycles of Eq. (126), for $F(t)=+B$ and $F(t)=-B$, will have uniform steady states that can be denoted $\phi_{ \pm}^{\prime}$ and $\phi_{ \pm}^{\prime \prime}$, respectively. Assuming $B \ll 1$, the escape times from the basins of attraction of these states can be determined approximately (Benzi et al., 1985),

$$
\begin{aligned}
& \tau_{+}^{\prime} \simeq C \exp \left\{2\left[\Delta \mathcal{F}\left(\phi_{+}\right)-B L a^{1 / 2}\right] / \sigma^{2}\right\}, \\
& \tau_{+}^{\prime \prime} \simeq C \exp \left\{2\left[\Delta \mathcal{F}\left(\phi_{+}\right)+B L a^{1 / 2}\right] / \sigma^{2}\right\},
\end{aligned}
$$

and similarly for $\tau_{-}^{\prime}$ and $\tau_{-}^{\prime \prime}$. Here $C$ is a constant independent of $\sigma^{2}$ and $\Delta \mathcal{F}\left(\phi_{+}\right)=\mathcal{F}\left(\phi^{(1)}\right)-\mathcal{F}\left(\phi_{+}\right)$measures the

\footnotetext{
${ }^{4} \mathrm{~A}$ more general spatiotemporal-dependent forcing function was originally proposed. Here, for simplicity, we only keep the time dependence.
}

minimum barrier height out of the basin of attraction of $\phi_{+}$in the landscape of the unforced free-energy functional,

$$
\mathcal{F}=\int d x\left[\frac{1}{4} \phi^{4}-\frac{1}{2} a \phi^{2}+\frac{\mathcal{D}}{2}(\nabla \phi)^{2}\right] .
$$

It has been demonstrated (Benzi et al., 1985) that $\phi^{(1)}(x)$ is the unstable steady state with the smallest free energy, and therefore this state is the one involved in the calculation of $\Delta \mathcal{F}\left(\phi_{+}\right)$.

Stochastic resonance will arise in this system if the escape times of a given steady state in each one of the driving periods are widely separated, with the smallest one being very small (so that the system jumps as soon as the external force changes its value), and the largest one being larger than the driving period (so that once the system jumps the first time, it remains in that well until the force changes value again). This is translated as $\tau_{+}^{\prime} \ll \pi / \omega$ and $\tau_{+}^{\prime \prime} \geq \pi / \omega$. Using Eqs. (127) and (128), those conditions translate in turn into limiting values for the noise intensity,

$$
\begin{aligned}
& \sigma_{\text {low }}^{2}=2\left[\Delta \mathcal{F}\left(\phi_{+}\right)-B L a^{1 / 2}\right] / \ln (\pi / C \omega), \\
& \sigma_{\text {high }}^{2}=2\left[\Delta \mathcal{F}\left(\phi_{+}\right)+B L a^{1 / 2}\right] / \ln (\pi / C \omega) .
\end{aligned}
$$

According to these results if $\sigma_{\text {low }}^{2} \ll \sigma^{2} \leq \sigma_{\text {high }}^{2}$, the system will be able to follow the external driving even if its amplitude is too low to elicit jumps in the deterministic case. The fact that the response of this spatially extended system to a periodic driving is enhanced at an intermediate range of noise levels is, once more, the hallmark of stochastic resonance.

Model (126) was also studied by Marchesoni et al. (1996) to provide an analytical framework for the phenomenon of array-enhanced stochastic resonance, which had been reported earlier (see the discussion above). On the basis of their analysis, the authors concluded that spatial coupling enhances the stochastic resonance phenomenon because the required activation energies are smaller in the spatial than in the homogeneous case. Indeed, in the presence of spatial degrees of freedom the system can jump between uniform stable steady states through nonuniform unstable (saddle) states $\phi^{(k)}(x)$, whose free energy is smaller than that of the unstable trivial solution $\phi_{0}=0$ (see above), this last one being the path necessarily taken by the corresponding zerodimensional system.

So far we have seen that spatial coupling enhances stochastic resonance in extended systems. But this is not always the case. Wio (1996) studied, for instance, a bistable reaction-diffusion system in the presence of an additive noise and an external modulated field applied only at the boundaries of the system. Using the concept of nonequilibrium potential, he concluded that in this case the signal-to-noise ratio does not depend noticeably on the coupling (diffusion) constant. When the method is applied to the case of a global (space-independent) harmonic forcing, on the other hand, the constructive influence of coupling (not only local but also nonlocal) is 

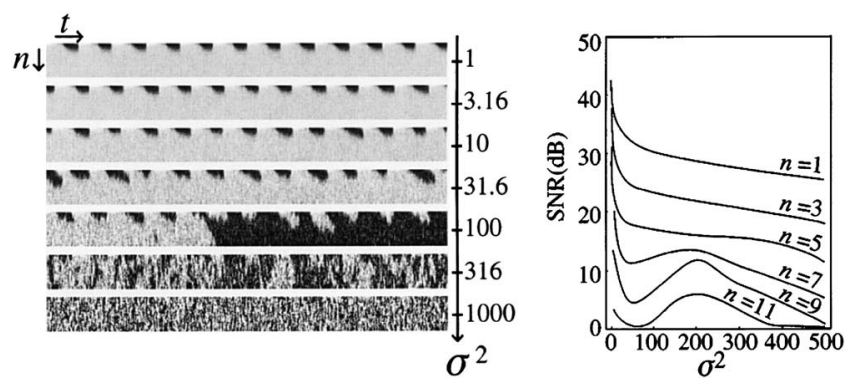

FIG. 32. Noise-enhanced propagation. Left: Spatiotemporal plots showing the response of a chain of 32 bistable oscillators to harmonic forcing of oscillator $n=1$ ( $n$ increases from top to bottom in each plot), and for increasing noise. The value of $\phi_{i}$ is represented in gray scale. Right: SNR versus noise intensity for different elements of the chain. Adapted from Lindner et al., 1998.

recovered (Castelpoggi and Wio, 1998). The nonequilibrium potential method has also been applied when the coupling coefficient depends on the state of the system, revealing a further enhancement of the SR effect with respect to a constant coupling (von Haeften et al., 2000).

Spatially extended stochastic resonance has also been studied in relation to other spatiotemporal phenomena, such as soliton motion (Gonzalez et al., 1998), front propagation (Dikshtein et al., 1998), decay of a metastable state (Nicolis et al., 1998), and pattern formation (Vilar and Rubí, 1997). In the latter, Vilar and Rubí (1997) applied an external harmonic driving to the SwiftHohenberg model, observing that noise helps the system amplify the temporal modulation and exhibit a periodic succession of homogeneous and patterned states. In a different development, Rabbiosi et al. (2003) showed theoretically that localized structures hinder the occurrence of stochastic resonance in spatially extended optical parametric oscillators. Hutt et al. (2002) developed a method to detect the signature of stochastic resonance in spatiotemporal systems.

\section{Noise-enhanced signal propagation}

Parallel to the study of stochastic resonance in extended media, there is also the question of the influence of noise on signal propagation. We start by considering the simplest situation, i.e., a bistable medium. Assume that the forcing term in Eq. (121) acts only upon a single oscillator in the chain. If the forcing amplitude is suprathreshold, the oscillator being directly modulated follows closely the input signal, but the propagation of such modulated signal down the chain should show interesting properties under noisy conditions. Numerical results by Lindner et al. (1998) are shown in Fig. 32. The results indicate that propagation of the periodic signal is optimal for an intermediate amount of noise: If noise is too small, only the first elements in the chain will oscillate with the forcing, the rest remaining quiescent because they are not being directly modulated. On the other hand, if noise is too large, all elements oscillate erratically. For an optimal amount of noise, the harmonic sig- nal reaches the farthest down the chain. The right panel in Fig. 32 shows the dependence of the SNR on the noise intensity for different elements of the chain. It is clear that for the oscillator that is directly modulated $(n=1)$, and those close to it, noise monotonously decreases the response at the input frequency. Oscillators farther downstream (such as $n=11$ ), on the other hand, exhibit the typical hallmark of stochastic resonance (a maximum in the SNR for a nonzero noise level), even though they are not being directly modulated. Hence, one can conclude that noise enhances the propagation of the harmonic signal through the system. Similar results were obtained numerically by Zhang et al. (1998) and Hauptmann et al. (1999) in arrays of one-way coupled bistable systems. An experimental realization in a line of electronic threshold elements, and the corresponding theoretical analysis (which is feasible in this case), were reported by Chapeau-Blondeau (1999) [see also Fuchikami and Sakaguchi (2003)].

The tolerance of this mechanism to "defects," i.e., sites in which noise is absent, was examined by Perazzo et al. (2000). Carusela et al. (2001), on the other hand, studied a possible use of a ring of unidirectionally coupled bistable elements as a short-term memory, by using noise-enhanced propagation of a sinusoidal signal as a mechanism to store digital information in the ring. Sendiña-Nadal and Pérez-Muñuzuri (2001) analyzed numerically the enhanced propagation of a periodic signal through a nonexcitable chemical medium described by the Oregonator model. Noise-enhanced propagation of two-frequency signals was studied by Zaikin, Topaj, et al. (2002), who reported nontrivial interactions between the two frequencies being propagated by noise. Ullner, Zaikin, García-Ojalvo, Báscones, and Kurths (2003) showed, both numerically and experimentally in an array of electronic circuits, that the role of noise could be played by a high-frequency forcing, in an example of noise-enhanced propagation via vibrational resonance. In a different context, Jiang and Xin (2000) showed that coupling enhances propagation of limit cycles induced by noise (via the mechanism of stochastic coherence) along a chain of unidirectionally coupled nonlinear oscillators [see also Postnov et al. (1999)].

Pulse propagation can also be mimicked in singlecomponent reaction-diffusion systems displaying bistability, in the form of kink-antikink pairs that propagate due to a convective term. Such signals travel with a fixed velocity but decay in the absence of noise [since the kink-antikink pair is structurally unstable (Habib and Lythe, 2000]. However, this process can be controlled including multiplicative noise, in such a way that the signal is able to propagate without distortion for optimal noise levels (García-Ojalvo et al., 2000). More complicated noise-sustained propagation phenomena arising in excitable media are described later.

The effect of thermal fluctuations on pulse propagation through a chain of masses coupled locally via anharmonic potentials was studied by Sarmiento et al. (1999). The speed of the pulses was seen to either increase or 
decrease with thermal noise, depending on the type of nonlinearity.

\section{Spatiotemporal stochastic resonance}

Following the notation introduced in the beginning of this section, spatial stochastic resonance under a purely spatially periodic signal was studied by Vilar and Rubí (2000) in a Ginzburg-Landau-like model, to assure bistability, supplemented with convection. In what remains of this section, we refer to spatiotemporal stochastic resonance as describing resonant effects mediated by noise in extended media forced with spatiotemporal signals rather than with pure temporal or spatial inputs.

A pioneering study in this respect was that by Jung and Mayer-Kress (1995b), who considered a twodimensional array of pulse coupled identical spiking threshold units with ad hoc refractory periods. A spatiotemporal white noise of zero mean was introduced to reproduce the effects of a thermal environment. The authors showed that an external wavelike perturbation elicited a resonant response in the form of a spatiotemporal propagating pattern with a sharp peak at a finite well-defined noise level, independent of the system size. More recently, this situation was revisited by Krawiecki et al. (2000) [see also Gabbay et al. (2004)], observing again a beneficial effect of coupling, which was nevertheless diminished due to the phase differences of the driving signal between neighboring elements. A subsequent study in two coupled discrete threshold elements (Krawiecki and Stemler, 2003) showed that this detrimental effect can in principle be compensated with delay in the coupling.

An experimental realization of the theoretical predictions of Jung and Mayer-Kress (1995b) was reported in the photosensitive version of the BZ reaction by Kádár et al. (1998). Their main experimental result is reproduced in Fig. 33. The figure shows, in the bottom row, a snapshot of the illumination profile, consisting of a (Gaussian) random square-patterned field, refreshed at regular time intervals and with average intensity chosen slightly below the threshold for sustained wave propagation. Forcing waves were successively originated in an adjacent region (at the left of the plots) with a lower level of illumination (and thus higher excitability) and directed into the observation area. As shown in Fig. 33(c), intermediate noise levels support the propagation of traveling waves. Not surprisingly, the effect turned out to depend on the cell size and, more importantly, on the noise refreshing time. In particular, wave propagation failed at both high and low refreshing frequencies, a signature of the delicate coupling between the noise and reaction-diffusion time scales.

\section{B. Autonomous systems}

Resonant behavior at intermediate noise intensities can also be observed in the absence of an external time scale. This effect popularly known as coherence reso-
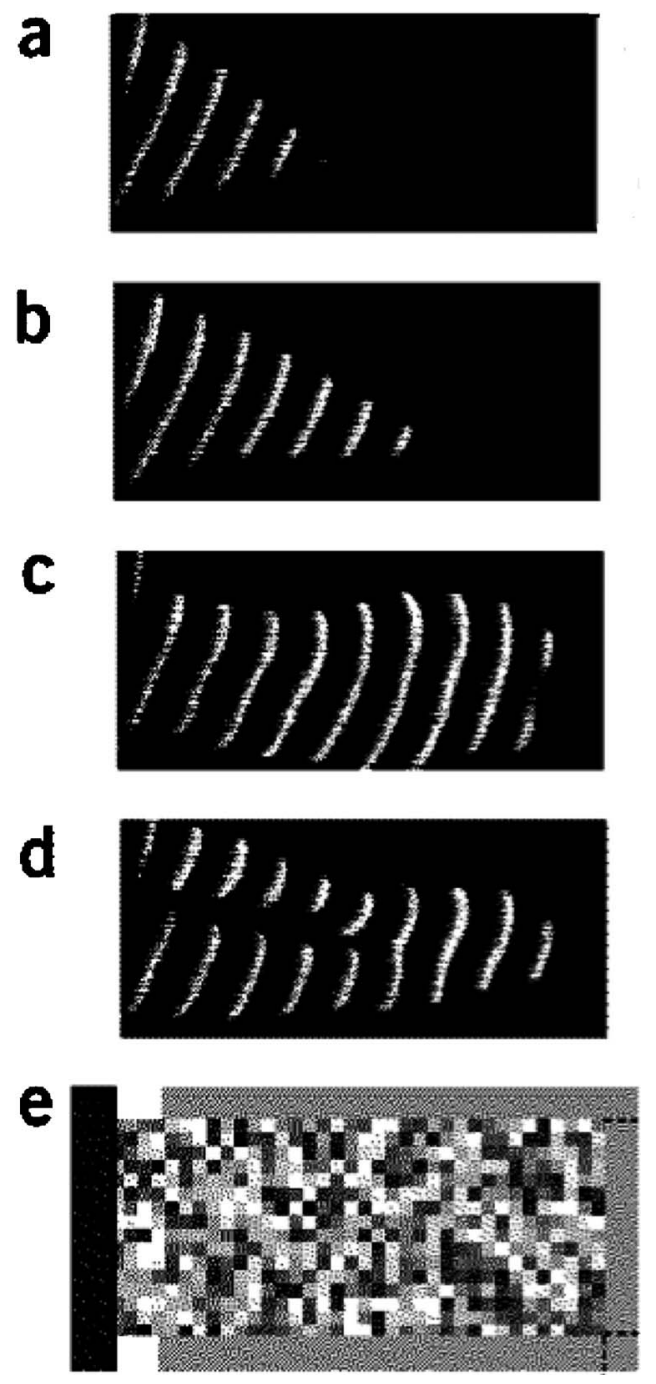

FIG. 33. Noise-supported waves in a subexcitable photosensitive BZ medium. (a)-(d) For increasing levels of noise, superimposed snapshots (taken at equal time intervals) of a single wavefront propagating from left to right. (e) A snapshot of the random illumination profile that provided the noisy driving. From Kádár et al., 1998.

nance, and that we have preferred to refer to as stochastic coherence (see Sec. I.D.3), will be reviewed here in spatially extended systems.

\section{Stochastic coherence in extended media}

Analogously to the developments described above in the field of spatially extended stochastic resonance, studies of stochastic coherence in extended systems were first restricted to globally coupled systems (Kurrer and Schulten, 1995; Rappel and Karma, 1996). Those studies, like most that followed, were often aimed at investigating the effect of internal noise on neuronal networks. Single-variable models (for a set of active rotators or referring to integrate-and-fire units, respectively, in the references cited above) were employed with the aim to extract as many analytical results as possible. Models of stochastic firing by randomly connected elements (Pham 


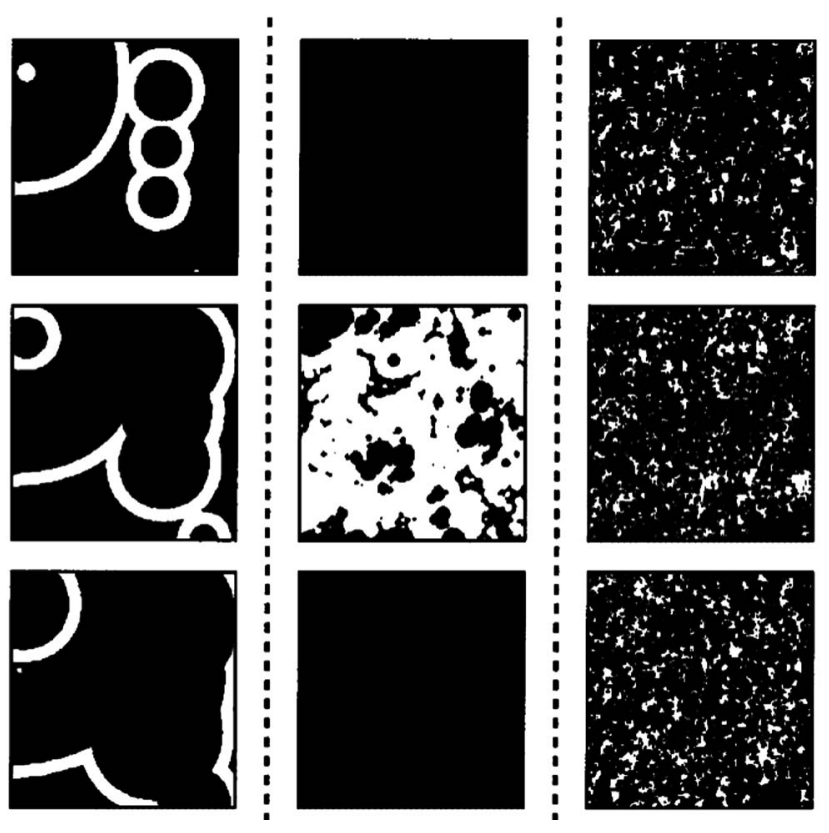

FIG. 34. Coherence resonance in an extended system. The dynamics of a two-dimensional array of FitzHugh-Nagumo elements is plotted at different time instants (from top to bottom) and for three noise levels: low (left column), intermediate (middle column) and high (right column). The quiescent state is represented in black, the excited state in white. From Neiman et al., 1999.

et al., 1998) or Hodgkin-Huxley globally coupled neurons (Wang et al., 2000) were considered subsequently. A more recent reference is that of Zaks et al. (2005). Interest focused on the possibility to observe noise-induced synchronized regimes as a signature of coherent neural activity.

We postpone the issue of synchronization to Sec. VII, while we concentrate here on analyzing conditions of enhanced stochastic coherence. To this end we refer to the most generic, two-variable, activator-inhibitor representations of excitable systems. Unless explicitly stated, in what follows in this section we consider local diffusivelike coupling.

Neiman et al. (1999) considered both one- and twodimensional arrays of nonidentical excitable units of FitzHugh-Nagumo type, driven by additive local noise of zero mean. Depending on the noise intensity and for a sufficiently large value of the coupling strength, three different dynamical regimes are found, as shown in Fig. 34. For small noise (left column), waves are randomly nucleated by noise and correlation between firing units only exists locally, and is limited to the mean time of wave propagation. Under intense noisy forcing (right column), cells fire everywhere uncorrelated and without any chance to construct any sort of spatiotemporally coherent structure. For an optimal noise level (middle column), the medium shows phase coherence in an oscillatory state that encompasses the most distant cells in the array. Different measures of stochastic synchronization, referred to both the phase and frequency variables, con- firm quantitatively this scenario of maximum coherence at intermediate noise intensity.

Nearly simultaneously, the occurrence of arrayenhanced stochastic coherence was reported by $\mathrm{Hu}$ and Zhou (2000). The model was identical to that of Neiman et al. (1999). Similarly to what occurred with AESR, the maximum of the coherence factor displays nonmonotonous behavior with respect to the coupling constant, the most pronounced enhancement occurring at intermediate values, whereas neutral improvement with respect to a single element is observed for large values of the coupling constant. Subsequently, Zhou et al. (2001) extended the model to introduce a certain degree of noise correlation among the different cells. Two interesting conclusions can be extracted from that numerical study: (i) noise correlation degrades coherence, and (ii) contrarily, inhomogeneities in the array parameters may enhance coherence (for intermediate coupling strength). Stochastic coherence was also reported in a lattice gas model of an oscillating reaction (Kortluke et al., 2002) and in small-world networks of excitable elements (Kwon and Moon, 2002; Kwon et al., 2005).

Finally, continuing the parallelism with stochastic resonance, system-size stochastic coherence was observed in globally coupled FitzHugh-Nagumo neural models (Toral et al., 2003). As in the case of SR (see above), the result admits a simple interpretation based on an effective renormalization of the noise intensity in terms of the square root of the number of elements in the array.

In a different development, Tsimring and Pikovsky (2001) had shown that excitablelike dynamics may arise in a discrete lattice as a combined effect of bistability and time delay. Subsequently, analytical and numerical studies soon reported the behavior of an ensemble of identical, noisy bistable units coupled through the timedelayed mean field (Huber and Tsimring, 2003, 2005). For positive feedback and above a noise-dependent (but delay-independent) threshold, the system undergoes an ordering second-order phase transition. For even larger, and also for negative enough, feedback strengths, the system displays multistability of oscillatory states whose regularity is maximal for a certain noise intensity, the signature of stochastic coherence. On the other hand, systems with time delay are somehow related to those with inhibitory coupling. This is precisely the idea put forward by Volkov, Stolyarov, et al. (2003). They analyzed the firing activity of a small number of FitzHughNagumo excitable units (two and three) that were coupled via diffusion of the inhibitor variable. In contrast to the standard form, the signature here of stochastic coherence is a noise-induced antiphase synchronization of excitable elements (Pikovsky et al., 2003).

Experimental confirmations of the above scenarios are practically nonexistent. The most closely related are those by Han et al. (1999), using monovibrator electronic circuits, who reported a small- (spatial) scale realization of stochastic coherence with a pair of identical "stochastic" oscillators. With similar equipment, the same authors detected more recently noise-induced multimode 
oscillatory behavior (Postnov et al., 2002). Propagation effects (noise only acting on the first unit of an array) were explored in a full-scale experiment by the same group (Postnov et al., 1999). Limited again to a pair of coherence-resonance oscillators, but this time in a BZ context, is the work by Ohtaki et al. (2004).

We close this subsection with a reference to frozen randomness, i.e., disorder. Disorder as a source of oscillations has been also considered (Cartwright, 2000; Boschi et al., 2001; Sosnovtseva et al., 2001). Diversity is claimed to provoke emergence of global oscillations from individual quiescent elements in heterogeneous excitable media.

\section{Noise-triggered waves and noise-mediated front propagation}

The perturbations required to activate an excitable system can originate from external signals, coupling from neighboring elements, or noise. In the absence of external driving, and in an original medium at rest, excitation waves can be nucleated only from noise. Recent analytical studies have addressed the conditions required for wave nucleation mediated by additive noise (Shardlow, 2004), and the corresponding nucleation rates (Henry and Levine, 2003), both studies conducted on FitzHugh-Nagumo-type models.

A more detailed although purely numerical study is that by Perc (2005), who used the Barkley model again to demonstrate spatial coherence (periodicity) on wave propagation triggered by additive noise. For a small intensity of the white noise, wave nucleation occurs scarcely. For very intense fluctuations, on the other hand, excitations arise very frequently and randomly. For an intermediate noise level, finally, nucleation times match on average the refractory time and the medium develops waves with a well-defined wavelength. Accordingly, an analysis of the structure function for increasing noise intensities shows an optimization of the spatial coherence of the system for intermediate noise levels. The wavelength unveiled by the noise depends on the diffusion coefficient $\mathcal{D}$, since $\sqrt{\mathcal{D}}$ controls the rate of diffusive spread. As this coefficient increases, the excitation travels farther during the excursion time, and correspondingly the optimal wavelength increases. The maximum wave number is seen to scale as $1 / \sqrt{\mathcal{D}}$ (Perc, 2005).

The effects of noise correlation parameters have been also considered: Busch and Kaiser (2003) studied numerically the case of an additive spatiotemporally correlated noise in the Barkley model and Beato et al. (2005) demonstrated in the photosensitive $\mathrm{BZ}$ reaction the existence of an optimal correlation time of the fluctuations that maximizes the nucleation coherence.

Beyond the phenomena of wave nucleation or propagation by noise, a number of numerical studies have shown that increasing amplitudes of random fluctuations are able to sustain different kinds of propagating structures, including spiral waves (Jung and Mayer-Kress, 1995a), spots and global oscillations (Hempel et al., 1999), or even well-organized targetlike patterns (Alonso et al., 2002). These studies, together with their experimental verification in the photosensitive BZ reaction (Wang et al., 1999; Alonso et al., 2001), are, however, representative of the effects of multiplicative noise, and we claim that their implications go beyond the additive noise-unveiled coherences discussed in this section. Thus we postpone their detailed consideration to Sec. VI.A, devoted to investigating genuine effects of parametric noise in establishing effective excitability conditions of spatially extended systems.

To finish this section, we refer briefly to noisemediated front propagation, an issue to be distinguished from the concept of noise-supported signal transmission discussed in Sec. V.A.2. In this respect the systems considered here are not supposed to be capable of repeated signal transmission in response to a continuously applied external input, as we assumed there. Rather, we consider noise-mediated, single (i.e., nonrepetitive) propagation of instantaneous disturbances, created either externally or by the noise itself. This idea was first developed experimentally by Löcher et al. (1998), who studied a onedimensional chain of symmetrically and locally coupled diode resonators operating in a biased bistable regime. Diffusive coupling and bias were chosen such that no propagation was possible in the absence of fluctuations, and amplified shot noise was independently applied at each element. The signal, triggered as an induced phase change at one end of the chain and measured at the other, was observed to propagate faster and more reliably for increasing values of the noise intensity, before being corrupted under intense noisy conditions. Later (Löcher et al., 2000) the situations of global versus local noise were compared, in both experiments and numerically. The averaged velocities of kink propagation turn out to be quite similar, as long as noise correlation is larger than the width front. To stress the differences between this scenario of facilitated transmission and that of array-enhanced stochastic resonance via synchronization, it is worth emphasizing the conclusion raised by the authors themselves when referring to the scaling of these behaviors with the number of elements in the array: contrary to improved signal-to-noise ratios for larger arrays, signal propagation here largely deteriorates when increasing the number of connected elements.

\section{Spatial stochastic coherence}

We have commented previously in Sec. IV.A that a phenomenon totally equivalent to stochastic coherence can also happen in space, with spatial frequencies, in the form of a pattern characterized by a wave vector $\mathbf{k}$, being revealed by noise. We now analyze this phenomenon, termed spatial stochastic coherence, as a function of the intensity of the (additive) noise.

Consider the Swift-Hohenberg equation (4), in the presence of an additive Gaussian noise $\xi(\mathbf{x}, t)$, white in space and time. As described in Sec. IV.A, in the absence of noise and for $a<0$ (below threshold), the system has a homogeneous stable steady state at $\phi=0$, representing conduction in the Rayleigh-Bénard cell. 

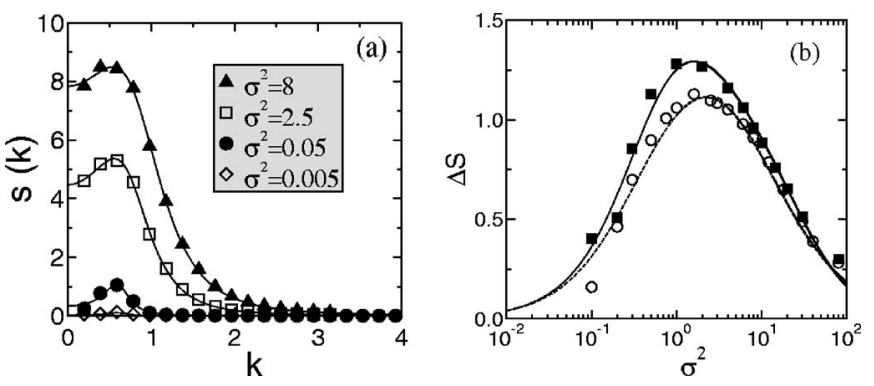

FIG. 35. Spatial stochastic coherence. (a) Structure function exhibited by the Swift-Hohenberg model (4) for $a=-0.005, k_{0}$ $=0.6$, and four different noise intensities listed in the legend. (b) $\Delta S$ vs noise intensity for two different values of the control parameter $a$. Squares correspond to $a=-0.005$ and circles to $a=-0.05$. In the two plots, lines correspond to functions (132) and (134), respectively, in terms of the renormalized parameter $a^{\prime}$. Simulations were performed in a square lattice of $64 \times 64$ cells with spacing $\Delta x=0.5$. From Carrillo et al., 2004.

Above threshold $(a>0)$, the system develops a roll-like pattern of nonzero wave number $k=k_{0}$, which represents convection. Below threshold $(a<0)$, and in the presence of a small amount of noise, the structure function can be calculated in steady state by linearizing Eq. (4) around $\phi=0$, and transforming the result to Fourier space, as described in Sec. IV.A. The result, given in Eq. (110), exhibits a well-defined spatial periodicity at $k=k_{0}$, as shown in Fig. 35(a). Moreover, the structure function (which is a spatial power spectral density) increases monotonically with the noise intensity. Of course, this calculation is no longer valid for large noise levels, because in that case the linearization around $\phi=0$ is not justified. In order to incorporate the nonlinear contributions of the noise, one can apply the Gaussian approximation (Langer, 1971) to the nonlinear term $\phi(x, t)^{3}$ $\sim 3 \phi(x, t)\left\langle\phi^{2}\right\rangle_{\mathrm{st}}$. Repeating the process outlined above, the resulting structure function is in this case

$$
S_{\mathrm{st}}(k)=\frac{\sigma^{2}}{\left(k^{2}-k_{0}^{2}\right)^{2}-a^{\prime}},
$$

with a rescaled linear parameter $a^{\prime} \equiv a-3\left\langle\phi^{2}\right\rangle_{\mathrm{st}}$. The average $\left\langle\phi^{2}\right\rangle_{\text {st }}$ can be evaluated consistently, resulting in $\left\langle\phi^{2}\right\rangle \sim\left(\sigma^{2}\right)^{2 / 3}$. Numerical evaluation of the effective parameter $a^{\prime}$ indicates that the dependence of $\sigma$ is well described by the Gaussian approximation (Carrillo et al., 2004).

As a measure of spatial coherence, we now define

$$
\Delta S \equiv S\left(k_{\max }\right)-S(0)
$$

[in numerical simulations a circular average of $S(\mathbf{k})$ is required]. Using Eq. (132) and the self-consistent average given above, we get

$$
\Delta S\left(\sigma^{2}\right)=-\frac{k_{0}^{4} \sigma^{2}}{a^{\prime}\left(k_{0}^{4}-a^{\prime}\right)} .
$$

This quantity does exhibit a maximum for a particular value of the noise intensity, as shown in Fig. 35(b). Such behavior is a clear indication that an intermediate noise level exists that enhances the spatial coherence in the system, analogously to what happens in temporal coherence resonance. The phenomenon occurs universally in pattern-forming systems; it has also been reported numerically in the chloride-iodine-malonic acid (CDIMA) reaction by Carrillo et al. (2004).

\section{SPATIOTEMPORAL DYNAMICS: NOISE-EFFECTIVE REGIMES}

Beyond the noise-unveiled resonances just discussed, fluctuations in spatially extended systems may have other striking dynamical effects. In this section, we concentrate on situations in which the interplay between nonlinearities and noise, mostly of a multiplicative origin, gives rise to well-defined transitions between different modes of self-organized behavior. Generically, we interpret these noise effects in terms of effective deterministiclike models of the kind referred to previously in Sec. II.B.3. In the first part of this section, we focus on excitable systems. Before presenting the essentials of this theoretical approach, we review the abundant experimental, analytical, and numerical literature accumulated during these last years on the subject. In the second part, doubly resonant effects will be discussed briefly as the paradigm of the combined effects of the noise-effective regimes discussed in this section and the noise-driven resonances analyzed in Sec. V.

\section{A. Multiplicative noise effects in excitable systems}

\section{Excitability transitions: Experimental and numerical realizations}

In this section, we review experimental and numerical results reported during the past decade on the effects of state-dependent noise in excitable systems. To get a better perspective of the successive developments of this topic, we have adopted a sort of historical order.

Although presented to the scientific community under the heading of spatiotemporal stochastic resonance, the first numerical evidence that wave propagation may be enhanced by noise was reported by Jung and MayerKress (1995b). As mentioned in Sec. V.A, those authors studied a two-dimensional pulse-coupled array of identical spiking threshold elements with spatiotemporal white noise. We stress here that the coupling strength was adjusted to be within the subexcitable regime, for which, in the absence of noise, excitable waves shrink as they propagate, and eventually die. Noise, within a certain range of intensities, was seen, however, to sustain coherent propagation. Although the noise appears in the model as pure additive (thermal-like), the way the spiking threshold is activated through pulse coupling renders the effect of noise effectively parametric. The model was exploited later on in relation to growth of spiral waves (Jung and Mayer-Kress, 1995a). These "thermal waves" (Jung, 1997) were statistically characterized in a following analysis using the concept of coherent space-time clusters of continued excitation. Their size distribution happened to satisfy a power law, which constitutes the 

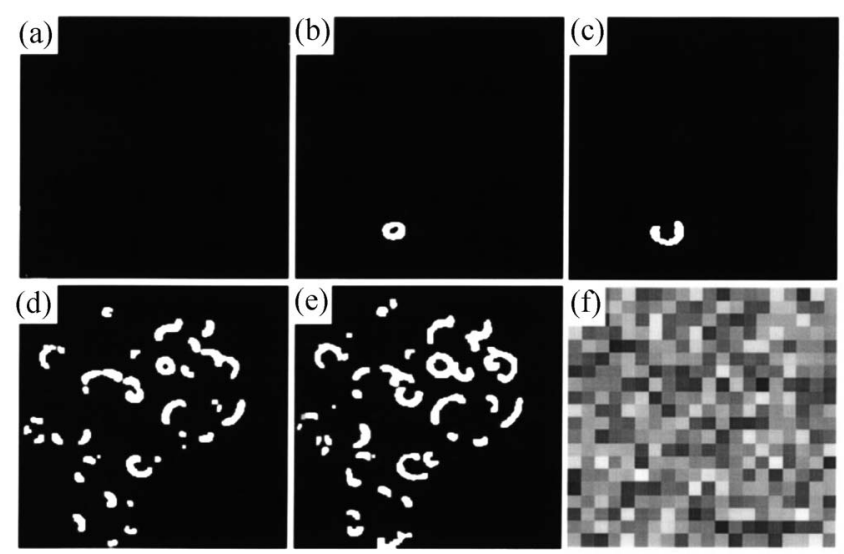

FIG. 36. Noise initiates and sustains waves in the subexcitable photosensitive BZ reaction. (a) The situation without noise, and (b),(c) and (d),(e) show two pairs of successive frames, at early and late stages of a wave initiation process under spatiotemporal noise, a snapshot of which is shown in (f). From Wang et al., 1999.

signature of a self-organized critical state. Later the same model was used to show that noise can induce complex scroll patterns in 3D media (Zhou and Jung, 2000).

Analogously, the experiment by Kádár et al. (1998), aimed also at verifying experimentally the phenomenon of spatiotemporal stochastic resonance as discussed in Sec. V.A, constitutes in fact the first demonstration of noise-enhanced excitability. Soon afterwards, the same group (Wang et al., 1999) used their experimental setup (a BZ photosensitive medium with Gaussian, zero-mean fluctuations in the forcing illumination) to look for the above-mentioned evidence of self-organized criticality. In those experiments, noise was indeed found to initiate and sustain wave behavior under subexcitable conditions, as shown in Fig. 36. A statistical analysis of the corresponding coherent space-time clusters led to a power-law distribution, and hence to the lack of any intrinsic length or time scale in the system, in consonance with the numerical prediction of Jung (1997), and featuring a typical avalanchelike behavior. In a different context, neurophysiological instead of chemical, but with similar aims, intercellular calcium signals were identified as noise-nucleated waves in cultured networks of rat hippocampal astrocytes by Jung et al. (1998).

Back to numerical simulations, the effects of fluctuations in the excitability threshold were studied by Hempel et al. (1999) using a detailed cellular automaton model with activator-inhibitor dynamics. Different regimes were found in terms of the noise intensity. In particular, nucleation of pulsating spots at intermediate noise levels turns into a synchronized state of global oscillations as the fluctuation intensity increased.

Noise-driven nucleation of waves has also been invoked as a mechanism of noise-induced memory in neuronal dendritic regions by Chialvo et al. (2000). The basis of such a mechanism is the interplay between stochastic activation, deterministic refractoriness, and finite speed
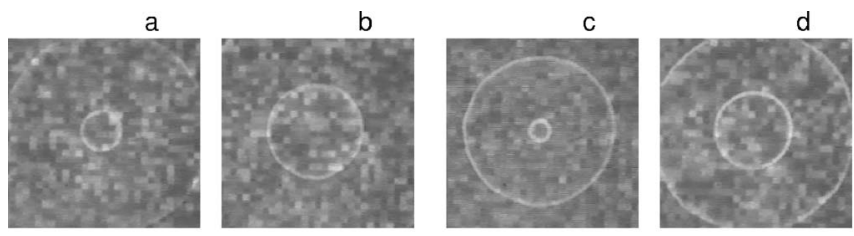

a
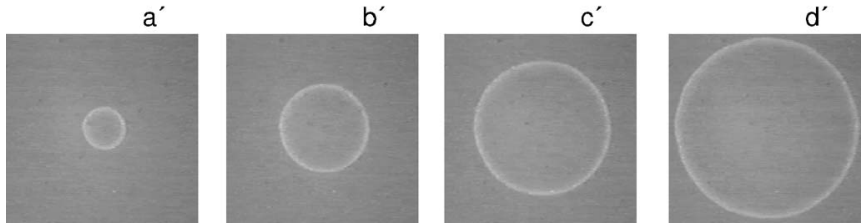

FIG. 37. A target pattern out of noise [(a)-(d), for increasing times] in an excitable (on average) light-sensitive BZ medium. For comparison, $\left(a^{\prime}\right)-\left(d^{\prime}\right)$ correspond to the noise-free case with a constant and uniform illumination fixed to the averaged intensity. From Alonso et al., 2001.

of wave propagation in excitable media. This combination causes an increased probability of activation in sites where the previous nucleation took place, which Chialvo et al. (2000) interpreted as a form of memory in a neural context. A similar mechanism lies behind the stochastic pacemakers studied by Jung and Gailey (2000).

Extending the observation of noise-enhanced wave propagation by Kádár et al. (1998), the experimental work by Alonso et al. (2001) demonstrated that not only waves can be sustained by noise in subexcitable conditions, but that regular pacemakers (a signature of sustained oscillatory activity) can also be created out of noise under conditions that are on average purely excitable. A distinctive plot of these experiments, conducted once more in the photosensitive BZ reaction, is shown in Fig. 37. Noise was introduced via a random illumination profile of squares uncorrelated in space and white in time, as set by the computer update operational time $(100 \mathrm{~ms})$. Both the experimental results for noisesupported waves in subexcitable conditions and noisecreated target patterns in nonoscillatory regimes were reproduced by numerical simulations of the generalized Oregonator model as it applies to the photosensitive BZ reaction (6) under multiplicative zero-mean Gaussian noise (Alonso et al., 2001).

More or less simultaneously, the limiting case of infinite spatial correlation (purely temporal noise) was studied experimentally by Zhou, Jia, et al. (2002) [see also Jia et al. (2004)] in the traditional (nonphotosensitive) BZ reaction, introducing this time the noise via a random electric field. In this case, synchronous nucleation of waves throughout the system was observed for an optimal noise level.

Finally, Zhou, Kurths, Neufeld, and Kiss (2003) examined numerically the interplay among additive noise, diffusion, and chaotic mixing in an excitable twodimensional FitzHugh-Nagumo model [see Eq. (26)]. This study revealed a regime of global oscillations at an intermediate noise level that depends on the flow rate. 

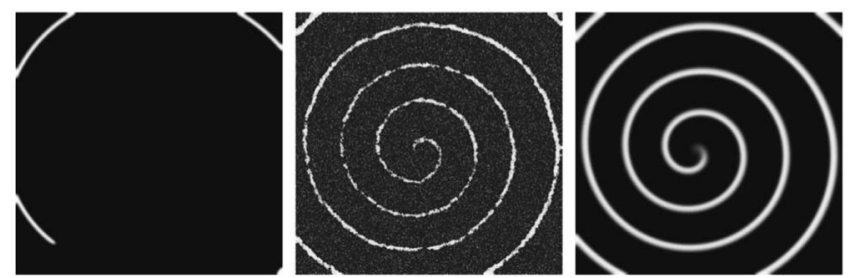

FIG. 38. A numerically obtained spiral sustained by noise (center), compared with the behavior of the corresponding deterministic effective model (right). In the absence of noise, the spiral dies away (left). Adapted from Alonso et al., 2002.

Actually, the behavior shown in Fig. 37 constitutes a particular example of a whole range of noise-induced transitions linking different excitability regimes, including subexcitable $\rightarrow$ excitable (the case of Fig. 33), excitable $\rightarrow$ oscillatory (the case of Fig. 37), bistable $\rightarrow$ excitable (García-Ojalvo et al., 2001; Báscones et al., 2002), and even a reverse transition oscillatory $\rightarrow$ excitable (Ullner, Zaikin, García-Ojalvo, and Kurths, 2003). These noise-induced transitions can be understood theoretically in terms of effective models, as described in the next subsection.

\section{Excitability transitions: Nullcline-based scenarios}

As an application of the general formalism presented in Sec. II.B.3, consider again the Barkley model

$$
\begin{aligned}
& \frac{\partial u}{\partial t}=\frac{1}{\epsilon} u(1-u)\left(u-\frac{v+b+\eta(\mathbf{r}, t)}{a}\right)+\mathcal{D}_{u} \nabla^{2} u, \\
& \frac{\partial v}{\partial t}=(u-v),
\end{aligned}
$$

under the action of a multiplicative spatiotemporal white noise in space and time with correlation given by the (two-dimensional) version of Eq. (31) and $c(0)$ $=\sigma^{2} /(\Delta x)^{2}$. The effective reaction-diffusion model is in this case particularly simple, since it amounts to a pure renormalization of the original Barkley model, with effective parameters (Alonso et al., 2002)

$$
\begin{aligned}
a^{\prime} & =a\left(1-\frac{2 c(0)}{\epsilon a^{2}}\right), \\
b^{\prime} & =b-\frac{c(0)}{\epsilon a}, \\
\epsilon^{\prime} & =\frac{\epsilon}{\left|1-2 c(0) / \epsilon a^{2}\right|} .
\end{aligned}
$$

The validity of this approach is qualitatively supported by Fig. 38, which compares a noise-induced spiral (center plot) with that generated by a deterministic effective model (right plot) with the parameters redefined according to Eq. (136). In this case noise is essential for the appearance of the spiral, since the medium is in a subexcitable regime and in the absence of noise any excita-
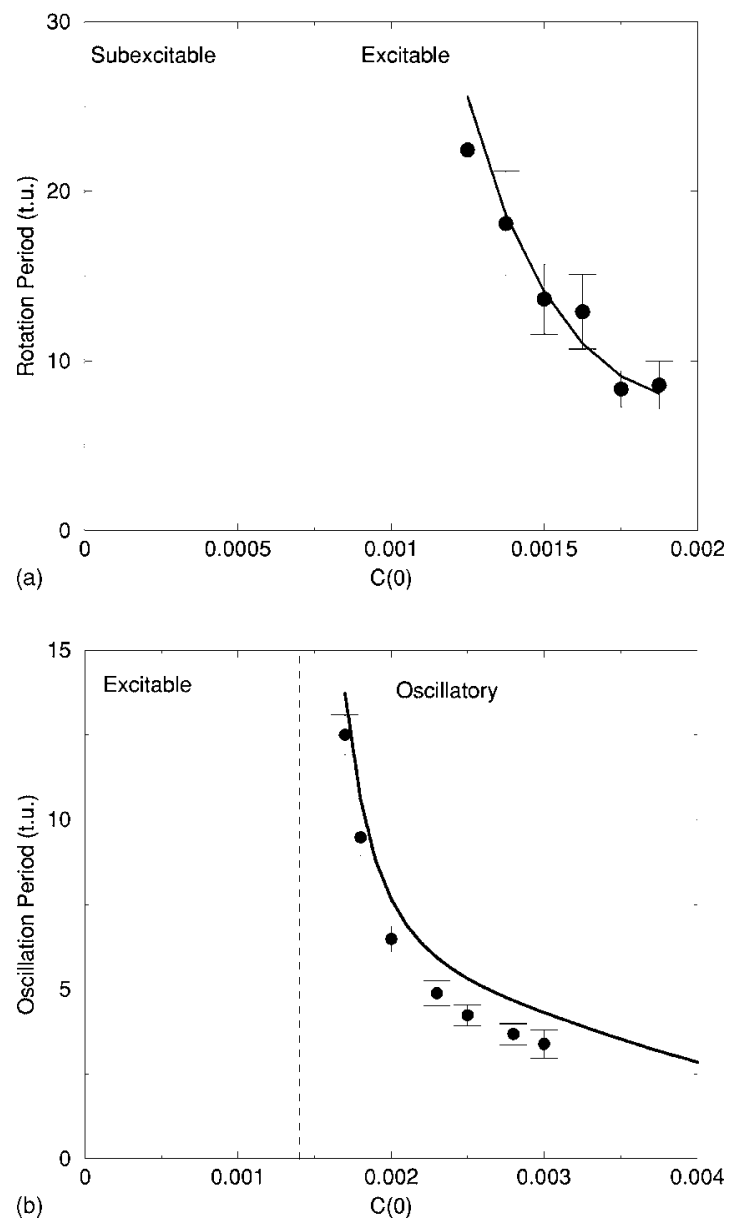

FIG. 39. Mean rotation period of a noise-sustained spiral (top) and mean oscillation period of a noise-sustained target (bottom) versus noise intensity. Continuous lines correspond in both cases to the results of the effective reaction-diffusion Barkley model. Vertical dashed lines represent the transitions between different dynamical regimes. From Alonso et al., 2002.

tion wave with free ends shrinks as it propagates, and eventually disappears.

The noise-induced spirals rapidly adopt a characteristic pitch and rotation period that depends on the noise intensity and is completely in agreement with the deterministic counterpart coming from the effective model, as shown in the top panel of Fig. 39. This is a distinctive signature of a multiplicative-noise effect, different from pseudo-oscillations arising in stochastic coherence, whose frequency is independent of the intensity of the additive noise that produces the phenomenon. The two plots in Fig. 39 also show, as vertical lines, the locations of the deterministic bifurcations that can be crossed by increasing the intensity of the parametric fluctuations (Alonso et al., 2002).

This theoretical methodology can also help in the understanding of noise-induced target patterns. A comparison between direct numerical simulations and effective model predictions, this time corresponding to the oscillation period of the target pattern, is shown in the bottom panel of Fig. 39. This theoretical analysis can be extended to encompass correlated fluctuations in both 
space and time, as shown by Alonso et al. (2002). Hou and Xin (2002) compared spatial, temporal, or spatiotemporal (white in space and time) parametric fluctuations in Barkley's model in relation to spiral wave formation.

\section{Other noise effects in active media}

Noise also has other nontrivial effects in active media. An example was provided by Hemming and Kapral (2000), who considered resonantly forced oscillatory systems, where the resonant forcing is applied to the system in a spatially inhomogeneous (random) way. The phenomena observed are diverse, ranging from roughening of the usual fronts separating phase-locked domains to unusual spiral wave dynamics. In a second example, noise-controlled self-replicated patterns have been reported numerically by Lesmes et al. (2003) in a simple autocatalytic reaction-diffusion system. Under intermediate intensities of additive white noise in both the activator and inhibitor variables, a noise-controlled transition from stripe growth to spot replication was reported, a behavior claimed to be reminiscent of cell colony formation in random environments.

\section{B. Doubly resonant effects}

The noise-induced phenomena discussed so far rely on a particular deterministic behavior of the underlying nonlinear system. When this required behavior is induced itself by a second source of noise, a doubly stochastic effect arises. Here the constructive role of fluctuations is twofold. For example, first a multiplicative noise can induce bistable behavior in a deterministically monostable medium, using the standard mechanisms of noise-induced phase transitions (see Sec. III); then a second, additive noise may enhance certain resonances in the response of this noise-induced bistable medium. The result is a doubly stochastic resonance. The same effect leads to signal propagation in monostable media and to a novel mechanism of stochastic coherence.

\section{Doubly stochastic resonance}

Consider a $d$-dimensional array of dynamical elements described by

$$
\begin{aligned}
\frac{d \phi_{i}}{d t}= & f\left(\phi_{i}\right)+g\left(\phi_{i}\right) \eta_{i}(t) \\
& +\frac{\mathcal{D}}{2 d} \sum_{j \in \operatorname{nn}(i)}\left(\phi_{j}-\phi_{i}\right)+A \cos \omega t+\xi_{i}(t),
\end{aligned}
$$

where the nonlinear functions $f(\phi)$ and $g(\phi)$ are given by Eq. (94). As shown in Sec. III.A.1, this system exhibits, in the absence of additive noise, an ordering phase transition induced by the multiplicative noise term. This transition consists in the loss of stability of the state $\phi$ $=0$, and the appearance of two new (symmetrical) stable steady states that coexist. It is natural to expect that in this (noise-induced) bistable situation, a second source

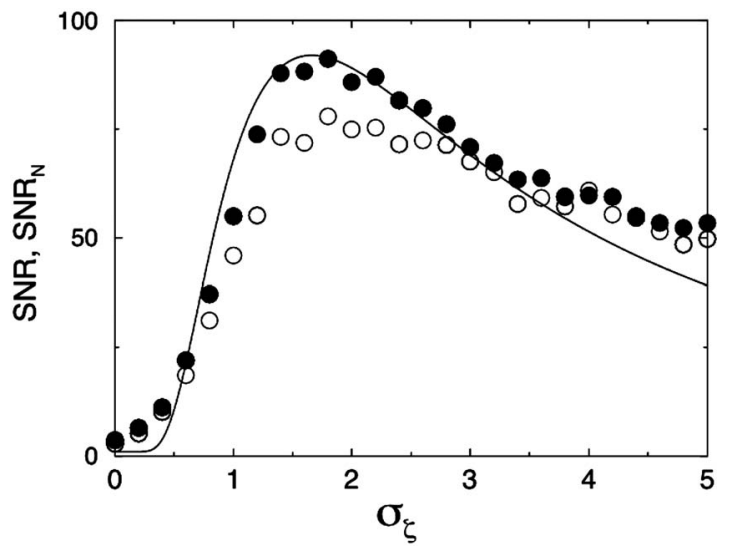

FIG. 40. SNR versus the intensity of the additive noise, for a nonzero value of the multiplicative noise. Symbols denote numerical simulation results of the field (filled circles) and its two-state approximation (empty circles), and the solid line the result of applying the linear-response theory to the corresponding stochastic potential of the system. From Zaikin et al., 2000 .

of noise will be able to produce a stochastic resonance effect. This doubly stochastic resonance was proposed and demonstrated by Zaikin et al. (2000) in the model system (137). Figure 40 shows the dependence of the SNR on the intensity of the additive noise, for a nonzero intensity of the multiplicative noise. The figure compares numerical simulation results with an analytical approach resulting from applying the standard linearresponse theory (Anishchenko et al., 2003) to the effective stochastic potential corresponding to the noiseinduced system (see Sec. II.B.2). It is worth emphasizing that the SNR is maximized in this case by tuning the values of the intensities of both noise terms.

The same mechanism has also been invoked by Pikovsky et al. (2002) to explain the existence of system size resonance in the noise-induced bistable medium described by Eq. (137), and by von Haeften et al. (2004) to show the appearance of stochastic resonance between noise-induced patterns (see Sec. IV).

\section{Signal propagation through monostable media}

We showed in Sec. V.A.2 that the same mechanism leading to array-enhanced stochastic resonance was able to sustain propagation of a periodic signal through an array of bistable elements. In the same way, and following the reasoning laid out above, a deterministically monostable medium, driven bistable by the action of a multiplicative noise, will support signal propagation enhanced by a second, additive source of noise. This was proven by Zaikin, García-Ojalvo, et al. (2002) in model (137), for a harmonic driving that affects only a small number of sites in an array of dynamical elements. As in the standard singly stochastic case (Sec. V.A.2), under suprathreshold harmonic modulation sites receiving the input will display a monotonic decrease in the SNR, while oscillators further down the chain exhibit the characteristic bell shape of the SNR in stochastic resonance. 
If, on the other hand, the input sites were subject to subthreshold periodic driving, all elements in the system exhibit a maximum in the SNR at an optimal noise level. In the absence of multiplicative noise, on the other hand, SNR decreases monotonically with noise in all situations, as corresponds to a monostable system. Again, the two noise sources need to be tuned simultaneously in this case in order to optimize propagation.

\section{Stochastic coherence via symmetry restoring}

So far we have described situations in which one of two noise sources induced bistability in a medium, and the second one produced a constructive effect under those conditions. But other types of doubly stochastic effects exist. For instance, another example is provided by activator-inhibitor media operating in a bistable regime. These media are able to exhibit stochastic coherence even in bistable conditions, because noise can induce jumps between both stable states in an equally effective basis, provided the basins of attraction of the two stable states are symmetric. In the general asymmetric situation, Zaikin et al. (2003) showed that an appropriately tuned multiplicative noise can restore the symmetry of the dynamics, and a second source of noise can then produce a coherence resonance effect. Two noise sources must be simultaneously tuned, but unlike the previous situations spatial coupling is not a requisite here, because symmetry restoring occurs independently of the system dimensionality.

\section{SYNCHRONIZATION AND CONTROL}

The concept of synchronization is often invoked when referring to noise effects in spatially extended systems. In fact, we have already referred to it when dealing with array-enhanced resonant phenomena in several parts of Sec. V. In this last section, we will first briefly discuss different aspects of synchronization from a twofold perspective: (i) in relation to a particular class of phase oscillator models, i.e., the so-called active rotators, that have characteristics of both excitable and oscillatory behavior; and (ii) by reviewing the abundant literature on synchronization of chaotic oscillators by means of noise. The last subsection is devoted to a somewhat related concept, that of the control of chaotic regimes by fluctuations. In our opinion that extremely counterintuitive scenario is the most appropriate one to finish this review devoted to highlighting the constructive effects of noise in self-organizing extended systems.

\section{A. Stochastic synchronization of active rotators}

In its origin, synchronization is a concept associated with periodic dynamical systems, and in its more standard formulation it refers to nonlinear oscillators. A particular benefit is gained when parametrizing the oscillatory dynamics in terms of a phase variable. This reduction is singularly rewarding when dealing with coupled ensembles (Kuramoto, 1981). A prototypic
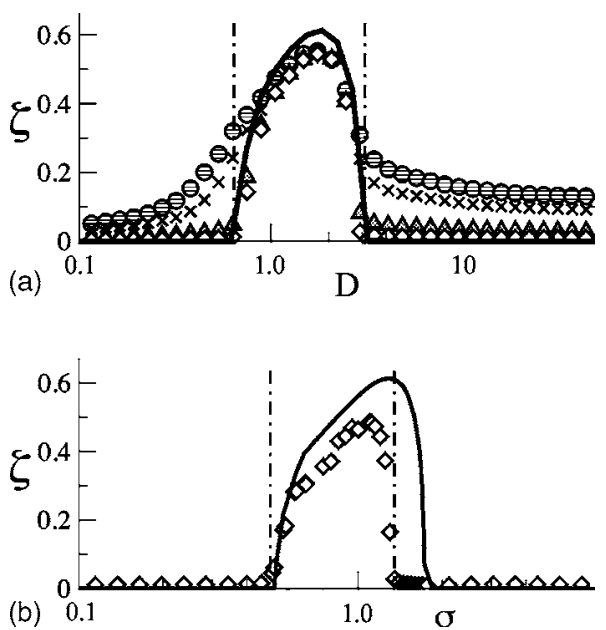

FIG. 41. Theoretical (solid lines) and numerical results for the degree of dynamic synchronization in terms of the noise intensity (top) and degree of diversity (bottom). Results are given in terms of the Shinomoto-Kuramoto order parameter $\zeta$, which is equal to 1 in the case of perfect dynamical (nonquiescent) synchronization. Adapted from Tessone et al., 2007.

model for a system of globally coupled active rotators under additive fluctuations is (Kurrer and Schulten, 1995; Zaks et al., 2003)

$$
\frac{d \phi_{i}(t)}{d t}=\omega-\sin \phi_{i}+\frac{C}{N} \sum_{j} \sin \left(\phi_{j}-\phi_{i}\right)+\xi_{i} .
$$

In this model, $\omega<1(\omega>1)$ corresponds to an excitable (oscillatory) regime of the $i$ th elementary rotator. Recently, Tessone et al. (2007) extended the model to encompass an eventual diversity in the individual frequencies of the elementary units. For identical units, Zaks et al. (2003) proposed the use of a Gaussian approximation for the mean field to solve the hierarchical dynamics of the collective modes in the ensemble, in that way extending to large noise intensities the earlier results obtained by Kurrer and Schulten (1995). By varying the magnitude of the stochastic forcing, three dynamical regimes were identified: stationary, rotatory, and remarkably a locally oscillatory state, called breathing. Tessone et al. (2007) focused on the purely excitable regime and developed a theory for the emergence of global firing conditions. A particular view of this phenomenon in terms of an appropriate order parameter measuring dynamic synchronization is plotted versus the intensity of noise and the diversity in Fig. 41. Numerical and meanfield theoretical results show that for intermediate values of these two parameters the ensemble exhibits synchronized firing. At the left of this region the oscillators are quiescent, and at the right firings are desynchronized.

\section{B. Stochastic synchronization of chaotic oscillators}

The first idea of synchronizing chaotic systems subjected to a common noisy input appeared formulated in the mid-1990s, and has been addressed since then in dif- 
ferent contexts, although using different names: it was called "reliability" in neurophysiology, to refer to the identical response of a neuron to a repeated noisy forcing (Mainen and Sejnowski, 1995), while the term "consistency" has been proposed recently in experiments with noise-driven ND: YAG lasers (Uchida et al., 2004). The phenomenon was primitively invoked for a single pair of identical units (Maritan and Banavar, 1994) [somewhat surprisingly, the apparently simpler idea of synchronizing nonchaotic oscillators was addressed quite later (Jensen, 1998)] and was immediately controversial. First the phenomenon was considered an artifact arising from a finite precision in numerical simulations (Pikovsky, 1994). Later it was attributed to a nonzero mean value of the noisy signal (Herzel and Freund, 1995; Malescio, 1996; Sánchez et al., 1997). More recently it has been demonstrated for zero-mean, additive Gaussian white noises of large enough intensity in certain chaotic maps (Lai and Zou, 1998; Toral et al., 2001) [the case with parametric noise was considered by Minai and Anand (1998)]. The generalization to the weaker level of phase synchronization was considered in the case of two nonidentical chaotic systems under common noise by Zhou and Kurths (2002a), and experimentally observed in noisy-neuronal oscillators by Neiman and Russell (2002).

Considering arrays of diffusively coupled identical, or nearly identical, chaotic units, a completely different concept was put forward by Lai and Liu (2001) [see also Zhou and Kurths (2002b)]. The authors proved that the temporal regularity of the system in terms of some measure of the synchronization between units is highly sensitive to noise resembling the phenomenon of stochastic coherence [in fact, the idea was formulated earlier for a single pair of Lorenz (Liu and Lai, 2001) and of Rössler (Zhou, Kurths, et al., 2002) oscillators]. Experimental verification, in the globally coupled case, was reported soon afterwards using an array of chaotic electrochemical oscillators (Kiss et al., 2003). Different possibilities to force an array of diffusively coupled chaotic units with parametric and/or additive noise forces, either white or time-correlated, were considered by Lorenzo and PérezMuñuzuri (2001), and implications in weather forecast methodologies have been recently discussed (Lorenzo et al., 2003).

\section{Control of chaos by noise}

Finally, we move from synchronization to control of chaotic systems, by considering first a scenario of spatiotemporal chaos and later a situation of threedimensional wave turbulence in weakly excitable systems.

\section{Taming spatiotemporal chaos}

Oscillator networks admit a representation in terms of complex Ginzburg-Landau dynamical units, and its globally coupled version was examined by Hakim and Rappel (1994) to look for effects of additive white noise. A

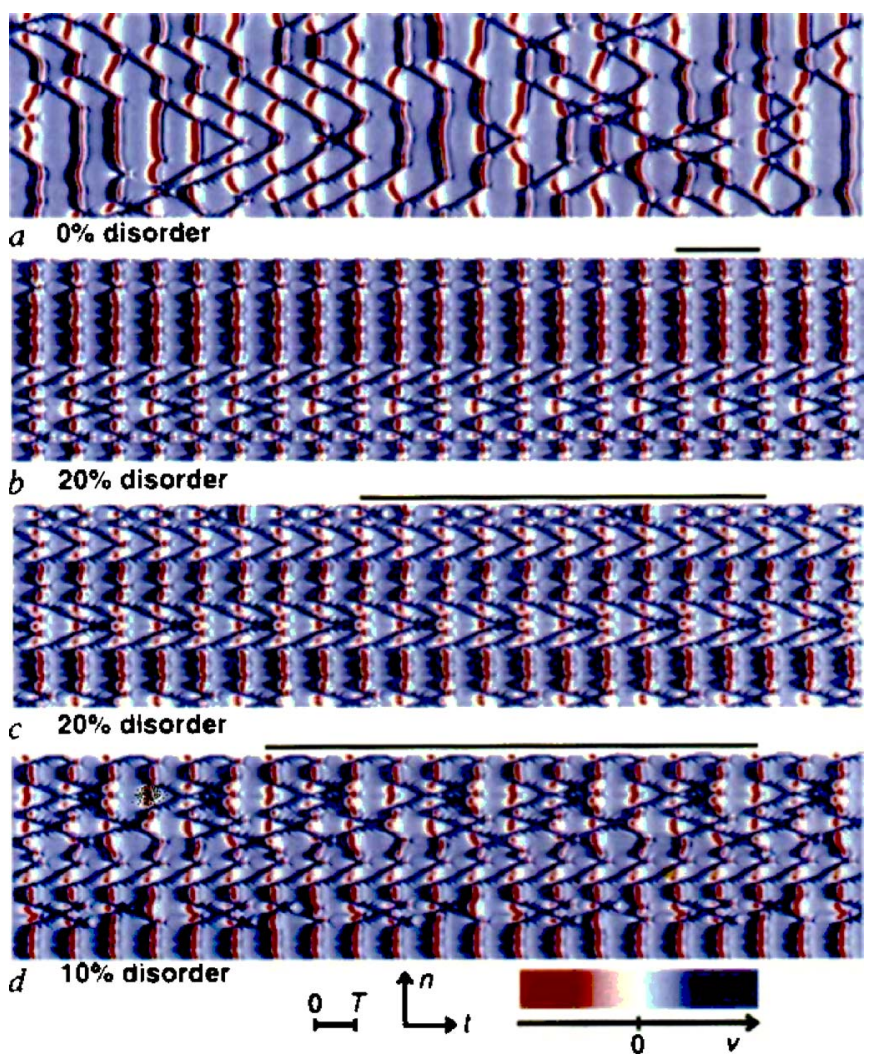

FIG. 42. (Color online) Spatiotemporal patterns of an array of 128 pendula for increasing levels of disorder. Color coding represents the angular velocity of the pendula, which are represented in the vertical direction, while time runs horizontally. (b),(c) Two different realizations of the same amount of frozen disorder. Horizontal black bars indicate the (b) length or (c),(d) half-length of periodic spatiotemporal patterns. From Braiman et al., 1995.

particular regime was chosen in which individual units follow erratic motions but all together combine in a coherent way to result in a nonvanishing average when increasing the number of oscillators. For weak noise, the mean-field amplitude remains nonzero and even an increase in the periodicity of the dynamics is found. For stronger intensities, a phase transition to a zero mean field is observed.

A slightly different idea, this time using frozen noise, i.e., disorder, was put forward by Braiman et al. (1995) as a strategy toward control of spatiotemporal chaos in (diffusively) coupled arrays of forced and damped nonlinear oscillators. In their numerical study, one- and twodimensional ensembles of chaotic units (forced nonlinear pendula in the simplest case considered, with a uniform distribution of their lengths) assemble into an ordered motion displaying complex but regular spatiotemporal patterns by frequency locking the motion to harmonics and subharmonics of the forcing. As quoted by these authors, the phenomenon appears in multiple systems and turns out to be robust with respect to both the parameter range and the size and realization of the disorder. Figure 42 shows the spatiotemporal evolution of an array of coupled pendula for different levels of 
disorder. Note that in the case of zero disorder a state of fully developed spatiotemporal chaos appears, while an optimal amount of disorder renders the dynamics regular. This behavior was reproduced experimentally by Shew et al. (1999).

\section{Controlling scroll-wave chaos}

In three dimensions, excitable media typically selforganize into wave structures known as "scroll waves" (Winfree, 1973). This phenomenology has not been as intensely scrutinized as spiral waves, due to both experimental difficulties (mainly at the control and monitoring level) and high computational demands. Scroll waves rotate around a one-dimensional singularity line known as a filament, which may be open (straight or curved), closed into a ring ("scroll rings"), or forming knots (Winfree and Strogatz, 1984). In particular, scroll rings are, unlike spirals, faintly robust structures and mostly collapse, although they may also expand under weakly excitable conditions (Panfilov and Rudenko, 1987). Moreover, expanding scroll rings are intrinsically unstable (Alonso, Kahler, et al., 2004) and eventually lead to wave chaos, sometimes invoked in relation to episodes of ventricular fibrillation in heart tissue (Fenton et al., 2002). For that reason, finding mechanisms that control the dynamical instabilities of 3D excitable waves is of strong interest. A promising strategy involves control via a spatially uniform and time-periodic (deterministic) forcing (Alonso et al., 2003).

Alternatively, numerical work has recently demonstrated rather counterintuitive taming effects, preventing scroll-wave turbulence, when applying a spatially distributed random external forcing to chaotic 3D wave propagation (Alonso, Sancho, et al., 2004). We review those results in what follows.

The numerical simulation of three-dimensional active media is an extremely demanding computational task. Therefore, in this particular context reaction-diffusion models must be chosen to be as simple as possible. As such, we study the effects of external fluctuations in the Barkley model (135), where multiplicative noise arises due to fluctuations in parameter $b$.

The typical scenario of scroll-wave control by noise is reproduced in Fig. 43. The figure shows how, under weakly excitable conditions, a 3D scroll wave develops an instability, through which its organizing (untwisted) filament starts to snake and bend around, giving rise to an entangled coil. The filament remains intact, though, until it reaches the boundary, at which point it breaks (Alonso, Kahler, et al., 2004). This regime is shown in the first four plots of Fig. 43. After introducing noisy forcing, the instability is eliminated and eventually the filament stretches out with superposed weak random displacements. As expected, this effect is possible only for a limited range of noise intensities, since under very intense noisy forcing waves would be randomly generated to compose a completely disorganized pattern of wave activity.

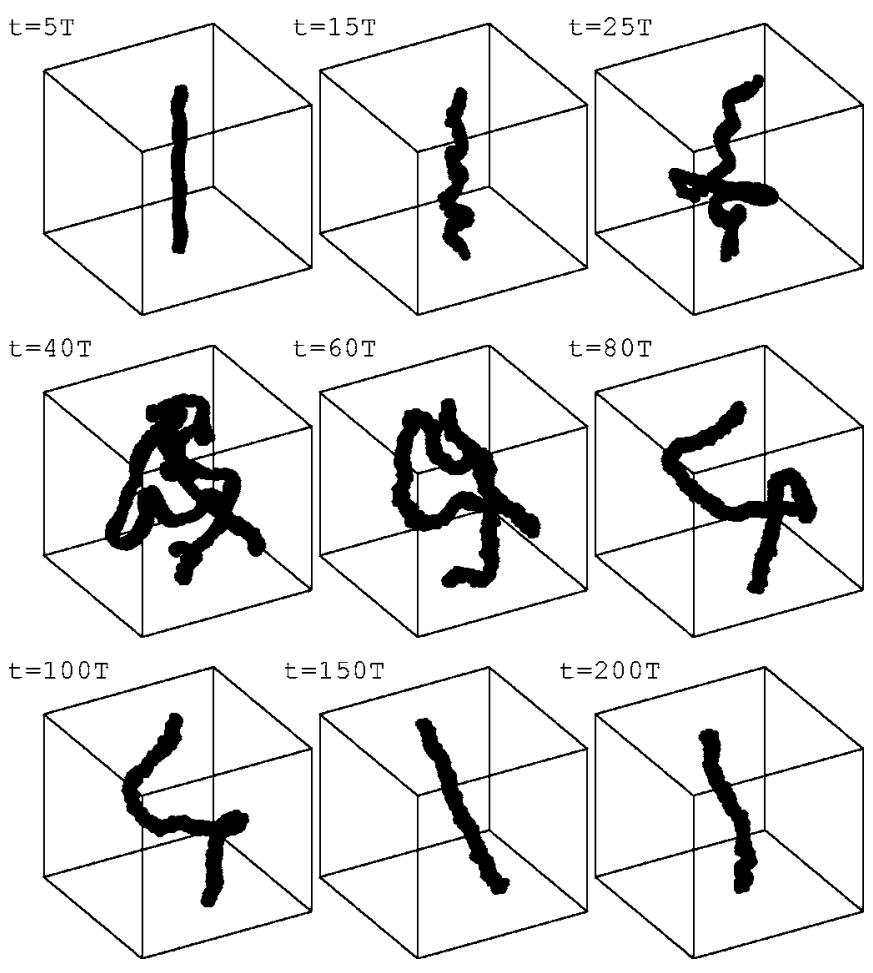

FIG. 43. Evolution of an initially unstable straight filament of a numerically simulated scroll wave. At time $t=40 T$, where $T$ is the wave rotation period, a random white noise forcing is added to the medium. From Alonso, Sancho, et al., 2004.

The key to understanding this behavior is rooted directly in the concept of negative filament tension, which underlies the mechanism of the scroll-wave instability under weak excitable conditions (Biktashev et al., 1994). In fact, as discussed in Sec. VI.A.2, the rationale to interpret the effect of multiplicative fluctuations relies on an effective modification of the medium excitability due to noise, which in turn leads to a renormalization of the tension of the scroll-wave filament. By choosing the model parameters appropriately, a condition of negative filament tension can be transformed into a positive one, thus taming scroll-wave turbulence. The opposite situation, when time-correlated noise stabilizes contracting scroll rings, was considered by Pérez-Muñuzuri et al. (2000).

\section{DISCUSSION}

Nature is noisy, and yet examples of self-organization abound. How can order arise out of such pervasive randomness? The simplest solution out of this seeming paradox is the possibility that self-organizing systems contain built-in mechanisms of robustness to noise (Barkai and Leibler, 2000). But a different, more appealing perspective is that in fact self-organizing mechanisms are built so as to employ unavoidable random fluctuations to their benefit. A large effort is being devoted by the scientific community to investigate the latter option. We have reviewed in this article examples of physical situations in which noise can be exploited to enhance, sus- 
tain, and induce spatially ordered behavior.

Historically, the first situations in which a constructive role of random fluctuations was identified in systems with spatial degrees of freedom corresponded to nonequilibrium phase transitions. That context allowed well-controlled and -defined predictions of the processes leading to spatiotemporal order out of noise. Unfortunately, no experiments have been performed to date that test these predictions. A different fate awaited the predictions of noise-ordering effects in pattern-forming and excitable media. In those systems, techniques such as the light-based control of certain nonlinear chemical reactions have allowed quantitative studies showing the undeniable constructive role of noise in extended media; a large part of this review has been devoted to those studies. Other experimental setups that have led to similar conclusions are coupled electronic arrays and optical systems.

The coming years should witness a wealth of theoretical and experimental results translating the knowledge outlined in this review to systems in the nanoscale, especially within the field of biology. Living systems are indeed outstanding in their ability to function in a reliable way in spite of being subject to a myriad of noise sources of non-negligible importance. Examples of these are the background synaptic noise acting upon brain neurons, caused by thousands of other neurons that are directly connected to them (Calvin and Stevens, 1967), and the biochemical noise arising in gene regulatory processes due to the finite (small) number of molecules involved in the corresponding biochemical reactions (Rosenfeld et al., 2005). In the latter context, for instance, predictions have been made, on the basis of theoretical models, that noise might play a constructive role in the accurate location of cell division in bacteria (Howard and Rutenberg, 2003). On the other hand, recent observations of the response of bacteria to stress have shown that excitable dynamics also arises at the level of gene regulation (Süel et al., 2006). This and other recent results open the way for a detailed investigation of the constructive influence of noise in spatially extended living systems.

Other small-scale systems where fluctuations are bound to play an essential role are offered by the recent surge of interest in nanotechnology. Coupled nanooscillators have already been observed to exhibit exciting instances of self-organization (Kaka et al., 2005). It is only a matter of time before the effects of random fluctuations, unavoidable in those types of systems, begin to be studied. Preliminary theoretical investigations (Stegemann et al., 2005) already exist.

Biology and nanotechnology provide beautiful examples of nonequilibrium (and nonlinear) dynamics where noise can be expected to have important effects. The techniques developed over the years to deal with those effects in physical systems, reviewed here, will help us to continue increasing our understanding of the mechanisms through which nonlinearities and noise symbiotically conspire to create order in nature.

\section{ACKNOWLEDGMENTS}

We thank all our colleagues who have collaborated with us in the study of noise-induced phenomena throughout the years. We also acknowledge financial support from the Ministerio de Educación y Ciencia (Spain) and FEDER, under projects FIS2006-03525 and FIS2006-11452, and from the Departament d'Universitats, Recerca i Societat de la Informació (DURSI) of the Generalitat de Catalunya, under projects 2005SGR-00653 and 2005SGR-00457. J.G.O. thanks the Generalitat de Catalunya and the European Commission (FP6-NEST project GABA) for additional financial support.

\section{REFERENCES}

Agez, G., C. Szwaj, E. Louvergneaux, and P. Glorieux, 2002, "Noisy precursors in one-dimensional patterns," Phys. Rev. A 66, 063805.

Alonso, S., R. Kahler, A. S. Mikhailov, and F. Sagués, 2004, "Expanding scroll rings and negative tension turbulence in a model of excitable media," Phys. Rev. E 70, 056201.

Alonso, S., F. Sagués, and A. S. Mikhailov, 2003, "Taming Winfree turbulence of scroll waves in excitable media," Science 299, 1722.

Alonso, S., F. Sagués, and J. M. Sancho, 2002, "Excitability transitions and wave dynamics under spatiotemporal structured noise," Phys. Rev. E 65, 066107.

Alonso, S., J. M. Sancho, and F. Sagués, 2004, "Suppression of scroll wave turbulence by noise," Phys. Rev. E 70, 067201.

Alonso, S., I. Sendiña-Nadal, V. Pérez-Muñuzuri, J. M. Sancho, and F. Sagués, 2001, "Regular wave propagation out of noise in chemical active media," Phys. Rev. Lett. 87, 078302.

Anishchenko, V. S., V. Astakhov, A. Neiman, T. Vadivasova, and L. Schimansky-Geier, 2003, Nonlinear Dynamics of Chaotic and Stochastic Systems: Tutorial and Modern Developments (Springer, Berlin).

Anishchenko, V. S., A. B. Neiman, F. Moss, and L. Schimansky-Geier, 1999, "Stochastic resonance: Noiseenhanced order," Phys. Usp. $42,7$.

Armero, J., J. Casademunt, L. Ramírez-Piscina, and J. M. Sancho, 1998, "Ballistic and diffusive corrections to front propagation in the presence of multiplicative noise," Phys. Rev. E 58, 5494.

Armero, J., J. M. Sancho, J. Casademunt, A. M. Lacasta, L. Ramírez-Piscina, and F. Sagués, 1996, "External fluctuations in front propagation," Phys. Rev. Lett. 76, 3045.

Barkai, N., and S. Leibler, 2000, "Circadian clocks limited by noise," Nature (London) 403, 267.

Barkley, D., 1991, "A model for fast computer simulation of waves in excitable media," Physica D 49, 61.

Barland, S., J. R. Tredicce, M. Brambilla, L. A. Lugiato, S. Balle, M. Giudici, T. Maggipinto, L. Spinelli, G. Tissoni, T. Knödl, M. Miller, and R. Jäger, 2002, "Cavity solitons as pixels in semiconductor microcavities," Nature (London) 419, 699.

Báscones, R., J. García-Ojalvo, and J. M. Sancho, 2002, "Pulse propagation sustained by noise in arrays of bistable electronic circuits," Phys. Rev. E 65, 061108.

Beato, V., I. Sendiña-Nadal, I. Gerdes, and H. Engel, 2005, "Noise-induced wave nucleations in an excitable chemical re- 
action," Phys. Rev. E 71, 035204(R).

Becker, A., and L. Kramer, 1994, "Linear stability analysis for bifurcations in spatially extended systems with fluctuating control parameter," Phys. Rev. Lett. 73, 955.

Behn, U., A. Lange, and T. John, 1998, "Electrohydrodynamic convection in liquid crystals driven by multiplicative noise: Sample stability," Phys. Rev. E 58, 2047.

Benzi, R., A. Sutera, and A. Vulpiani, 1981, "The mechanism of stochastic resonance," J. Phys. A 14, L453.

Benzi, R., A. Sutera, and A. Vulpiani, 1985, "Stochastic resonance in the Landau-Ginzburg equation," J. Phys. A 18, 2239.

Berthet, R., A. Petrossian, S. Residori, B. Roman, and S. Fauve, 2003, "Effect of multiplicative noise on parametric instabilities," Physica D 174, 84.

Bezrukov, S. M., and I. Vodyanoy, 1995, "Noise-induced enhancement of signal transduction across voltage-dependent ion channels," Nature (London) 378, 362.

Bielawski, S., C. Szwaj, C. Bruni, D. Garzella, G. L. Orlandi, and M. E. Couprie, 2005, "Advection-induced spectrotemporal defects in a free-electron laser," Phys. Rev. Lett. 95, 034801.

Biktashev, V. N., A. V. Holden, and H. Zhang, 1994, "Tension of organizing filaments of scroll waves," Philos. Trans. R. Soc. London, Ser. A 347, 611.

Boschi, C. D. E., E. Louis, and G. Ortega, 2001, “Triggering synchronized oscillations through arbitrarily weak diversity in close-to-threshold excitable media," Phys. Rev. E 65, 012901.

Braiman, Y., J. F. Lindner, and W. L. Ditto, 1995, “Taming spatiotemporal chaos with disorder," Nature (London) 378, 465.

Brand, H. R., S. Kai, and S. Wakabayashi, 1985, "External noise can suppress the onset of spatial turbulence," Phys. Rev. Lett. 54, 555.

Breskin, I., J. Soriano, E. Moses, and T. Tlusty, 2006, "Percolation in living neural networks," Phys. Rev. Lett. 97, 188102.

Buceta, J., M. Ibañes, J. M. Sancho, and K. Lindenberg, 2003, "Noise-driven mechanism for pattern formation," Phys. Rev. E 67, 021113.

Buceta, J., and K. Lindenberg, 2004, "Comprehensive study of phase transitions in relaxational systems with field-dependent coefficients," Phys. Rev. E 69, 011102.

Buldú, J. M., C. M. González, J. Trull, M. C. Torrent, and J. García-Ojalvo, 2005, "Coupling-mediated ghost resonance in mutually injected lasers," Chaos 15, 013103.

Buldú, J. M., R. Vicente, T. Pérez, C. R. Mirasso, M. C. Torrent, and J. García-Ojalvo, 2002, "Periodic entrainment of power dropouts in mutually coupled semiconductor lasers," Appl. Phys. Lett. 81, 5105.

Bulsara, A. R., and G. Schmera, 1993, "Stochastic resonance in globally coupled nonlinear oscillators," Phys. Rev. E 47, 3734.

Busch, H., and F. Kaiser, 2003, "Influence of spatiotemporally correlated noise on structure formation in excitable media," Phys. Rev. E 67, 041105.

Calvin, W. H., and C. F. Stevens, 1967, "Synaptic noise as a source of variability in the interval between action potentials.," Science 155, 842.

Cardy, J. L., and R. L. Sugar, 1980, "Directed percolation and Reggeon field theory," J. Phys. A 13, L423.

Carrillo, O., M. Ibañes, J. García-Ojalvo, J. Casademunt, and J. M. Sancho, 2003, "Intrinsic noise-induced phase transitions: Beyond the noise interpretation," Phys. Rev. E 67, 046110.

Carrillo, O., M. Ibañes, and J. M. Sancho, 2002, "Noise induced phase transitions by nonlinear instability mechanism,"
Fluct. Noise Lett. 2, L1.

Carrillo, O., M. A. Santos, J. García-Ojalvo, and J. M. Sancho, 2004, "Spatial coherence resonance near pattern-forming instabilities," Europhys. Lett. 65, 452.

Carroll, T. L., and L. M. Pecora, 1991, "Synchronizing chaotic circuits," IEEE Trans. Circuits Syst. 38, 453.

Cartwright, J. H. E., 2000, "Emergent global oscillations in heterogeneous excitable media: The example of pancreatic B cells," Phys. Rev. E 62, 1149.

Carusela, M. F., R. P. J. Perazzo, and L. Romanelli, 2001, "Stochastic resonant memory storage device," Phys. Rev. E 64, 031101.

Castelpoggi, F., and H. S. Wio, 1998, "Stochastic resonant media: Effect of local and nonlocal coupling in reactiondiffusion models," Phys. Rev. E 57, 5112.

Castets, V., E. Dulos, J. Boissonade, and P. De Kepper, 1990, "Experimental evidence of a sustained standing Turing-type nonequilibrium chemical pattern," Phys. Rev. Lett. 64, 2953.

Chandrasekhar, S., 1981, Hydrodynamic and Hydromagnetic Stability (Dover, New York).

Chapeau-Blondeau, F., 1999, "Noise-assisted propagation over a nonlinear line of threshold elements," Electron. Lett. 35, 1055.

Chialvo, D. R., G. A. Cecchi, and M. O. Magnasco, 2000, "Noise-induced memory in extended excitable systems," Phys. Rev. E 61, 5654.

Chialvo, D. R., A. Longtin, and J. Muller-Gerking, 1997, "Stochastic resonance in models of neuronal ensembles," Phys. Rev. E 55, 1798.

Ciliberto, S., E. Pampaloni, and C. Perez-Garcia, 1988, "Competition between different symmetries in convective patterns," Phys. Rev. Lett. 61, 1198.

Clerc, M. G., C. Falcon, and E. Tirapegui, 2005, "Additive noise induces front propagation," Phys. Rev. Lett. 94, 148302. Collins, J. J., C. C. Chow, and T. T. Imhoff, 1995, "Stochastic resonance without tuning," Nature (London) 376, 236.

Costa, J. M., F. Sagués, and M. Vilarrasa, 1991, "Fractal patterns from corrosion pitting," Corros. Sci. 32, 665.

Cross, M. C., and P. C. Hohenberg, 1993, "Pattern formation outside of equilibrium," Rev. Mod. Phys. 65, 851.

de Pasquale, F., J. Gorecki, and J. Popielawski, 1992, "On the stochastic correlations in a randomly perturbed chemical front," J. Phys. A 25, 433.

Dickman, R., 1994, "Numerical study of a field theory for directed percolation," Phys. Rev. E 50, 4404.

Dikshtein, I., A. Neiman, and L. Schimansky-Geier, 1998, "Stochastic resonance of front motion in inhomogeneous media," Phys. Lett. A 246, 259.

Dinklage, A., C. Wilke, and T. Klinger, 1999, "Spatio-temporal response of stochastic resonance in an excitable discharge plasma," Phys. Plasmas 6, 2968.

Dutta, S., S. S. Riaz, and D. S. Ray, 2005, "Noise-induced instability: An approach based on higher-order moments," Phys. Rev. E 71, 036216.

Falcke, M., 2003, "On the role of stochastic channel behavior in intracellular Ca2+ dynamics," Biophys. J. 84, 42.

Feinerman, O., M. Segal, and E. Moses, 2005, "Signal propagation along unidimensional neuronal networks," J. Neurophysiol. 94, 3416.

Fenton, F. H., E. M. Cherry, H. M. Hastings, and S. J. Evans, 2002, "Multiple mechanisms of spiral wave breakup in a model of cardiac electrical activity," Chaos 12, 852.

Field, R. J., E. Koros, and P. M. Noyes, 1972, "Oscillations in 
chemical systems II. Thorough analysis of temporal oscillations in the bromate-cerium-malonic acid system," J. Am. Chem. Soc. 94, 8649.

Frankowicz, M., A. L. Kawczynski, and J. Gorecki, 1991, "Stochastic effects in propagation of impulses: The BelousovZhabotinskii reaction," J. Phys. Chem. 95, 1265.

Fuchikami, N., and T. Sakaguchi, 2003, "Noise-induced transmission of signals through a chain of level-crossing detectors," Fluct. Noise Lett. 3, L11.

Gabbay, M., M. L. Larsen, and L. S. Tsimring, 2004, "Globally coupled noisy oscillators with inhomogeneous periodic forcing," Phys. Rev. E 70, 066212.

Gade, P. M., R. Rai, and H. Singh, 1997, "Stochastic resonance in maps and coupled map lattices," Phys. Rev. E 56, 2518.

Gammaitoni, L., P. Hänggi, P. Jung, and F. Marchesoni, 1998, "Stochastic resonance," Rev. Mod. Phys. 70, 223.

Gang, H., T. Ditzinger, C. Ning, and H. Haken, 1993, "Stochastic resonance without external periodic force," Phys. Rev. Lett. 71, 807.

Gang, H., H. Haken, and X. Fagen, 1996, "Stochastic resonance with sensitive frequency dependence in globally coupled continuous systems," Phys. Rev. Lett. 77, 1925.

Ganopolski, A., and S. Rahmstorf, 2002, "Abrupt glacial climate changes due to stochastic resonance," Phys. Rev. Lett. 88, 038501.

Gao, Z., B. Hu, and G. Hu, 2002, "Stochastic resonance of small-world networks," Phys. Rev. E 65, 016209.

García-Ojalvo, J., A. Hernández-Machado, and J. M. Sancho, 1993, "Effects of external noise on the Swift-Hohenberg equation," Phys. Rev. Lett. 71, 1542.

García-Ojalvo, J., A. M. Lacasta, F. Sagués, and J. M. Sancho, 2000, "Noise-sustained signal propagation," Europhys. Lett. 50, 427.

García-Ojalvo, J., J. M. R. Parrondo, J. M. Sancho, and C. Van den Broeck, 1996, "Reentrant transition induced by multiplicative noise in the time-dependent Ginzburg-Landau model," Phys. Rev. E 54, 6918.

García-Ojalvo, J., F. Sagués, J. M. Sancho, and L. ShimanskyGeier, 2001, "Noise-enhanced excitability in bistable activator-inhibitor media,” Phys. Rev. E 65, 011105.

García-Ojalvo, J., and J. M. Sancho, 1994, "Colored noise in spatially extended systems,” Phys. Rev. E 49, 2769.

García-Ojalvo, J., and J. M. Sancho, 1996, "External fluctuations in a pattern-forming instability," Phys. Rev. E 53, 5680.

García-Ojalvo, J., and J. M. Sancho, 1999, Noise in Spatially Extended Systems (Springer-Verlag, New York).

García-Ojalvo, J., J. M. Sancho, and L. Ramírez-Piscina, 1992, "A nonequilibrium phase transition with colored noise," Phys. Lett. A 168, 35.

Gard, T. C., 1987, Introduction to Stochastic Differential Equations (Dekker, New York).

Gardiner, C. W., 1989, Handbook of Stochastic Methods, 2nd ed. (Springer, Berlin).

Genovese, W., M. A. Muñoz, and J. M. Sancho, 1998, "Nonequilibrium transitions induced by multiplicative noise," Phys. Rev. E 57, R2495.

Gluckman, B. J., T. I. Netoff, E. J. Neel, W. L. Ditto, M. L. Spano, and S. J. Schiff, 1996, "Stochastic resonance in a neuronal network from mammalian brain," Phys. Rev. Lett. 77, 4098.

Goldman, D. I., J. B. Swift, and H. L. Swinney, 2004, "Noise, coherent fluctuations, and the onset of order in an oscillated granular fluid," Phys. Rev. Lett. 92, 174302.
Gollub, J. P., and J. F. Steinman, 1980, "External noise and the onset of turbulent convection," Phys. Rev. Lett. 45, 551.

Gonzalez, J. A., B. A. Mello, L. I. Reyes, and L. E. Guerrero, 1998, "Resonance phenomena of a solitonlike extended object in a bistable potential," Phys. Rev. Lett. 80, 1361.

González-Cinca, R., L. Ramírez-Piscina, J. Casademunt, and A. Hernández-Machado, 2001, "Sidebranching induced by external noise in solutal dendritic growth," Phys. Rev. E 63, 051602 .

Grinstein, G., M. A. Muñoz, and Y. Tu, 1996, "Phase structure of systems with multiplicative noise," Phys. Rev. Lett. 76, 4376.

Gunton, J. D., M. San Miguel, and P. S. Sahni, 1983, "The dynamics of first-order phase transitions," in Phase Transitions and Critical Phenomena, edited by C. Domb and J. Lebowitz (Academic, New York), Vol. 8, p. 267.

Habib, S., and G. Lythe, 2000, "Dynamics of kinks: Nucleation, diffusion, and annihilation," Phys. Rev. Lett. 84, 1070.

Hakim, V., and W. J. Rappel, 1994, "Noise-induced periodic behaviour in the globally coupled complex Ginzburg-Landau equation," Europhys. Lett. 27, 637.

Han, S. K., T. G. Yim, D. E. Postnov, and O. V. Sosnovtseva, 1999, "Interacting coherence resonance oscillators," Phys. Rev. Lett. 83, 1771.

Hauptmann, C., F. Kaiser, and C. Eichwald, 1999, "Signal transfer and stochastic resonance in coupled nonlinear systems," Int. J. Bifurcation Chaos Appl. Sci. Eng. 9, 1159.

Hemming, C. J., and R. Kapral, 2000, "Resonantly forced inhomogeneous reaction-diffusion systems," Chaos 10, 720.

Hempel, H., L. Schimansky-Geier, and J. García-Ojalvo, 1999,

"Noise-sustained pulsating patterns and global oscillations in subexcitable media," Phys. Rev. Lett. 82, 3713.

Henry, H., and H. Levine, 2003, "Wave nucleation rate in excitable systems in the low noise limit," Phys. Rev. E 68, 031914.

Herzel, H., and J. Freund, 1995, "Chaos, noise and synchronization revisited," Phys. Rev. E 52, 3238.

Hohenberg, P. C., and B. I. Halperin, 1977, "Theory of dynamic critical phenomena," Rev. Mod. Phys. 49, 435.

Hohenberg, P. C., and J. B. Swift, 1992, "Effects of additive noise at the onset of Rayleigh-Bénard convection," Phys. Rev. A 46, 4773.

Hong, H., H. Park, and M. Y. Choi, 2005, "Collective synchronization in spatially extended systems of coupled oscillators with random frequencies," Phys. Rev. E 72, 036217.

Horsthemke, W., and R. Lefever, 1984, Noise-Induced Transitions (Springer, Berlin).

Hou, Z., and H. Xin, 2002, "Noise-sustained spiral waves: Effect of spatial and temporal memory," Phys. Rev. Lett. 89, 280601.

Howard, M., and A. D. Rutenberg, 2003, "Pattern formation inside bacteria: Fluctuations due to the low copy number of proteins," Phys. Rev. Lett. 90, 128102.

$\mathrm{Hu}, \mathrm{B}$., and C. Zhou, 2000, "Phase synchronization in coupled nonidentical excitable systems and array-enhanced coherence resonance," Phys. Rev. E 61, R1001.

Huber, D., and L. S. Tsimring, 2003, "Dynamics of an ensemble of noisy bistable elements with global time delayed coupling," Phys. Rev. Lett. 91, 260601.

Huber, D., and L. S. Tsimring, 2005, "Cooperative dynamics in a network of stochastic elements with delayed feedback," Phys. Rev. E 71, 036150.

Hutt, M.-T., R. Neff, H. Busch, and F. Kaiser, 2002, "Method 
for detecting the signature of noise-induced structures in spatiotemporal data sets," Phys. Rev. E 66, 026117.

Hwang, S.-M., K.-H. Yea, and K. J. Lee, 2004, "Regular and alternant spiral waves of contractile motion on rat ventricle cell cultures," Phys. Rev. Lett. 92, 198103.

Ibañes, M., 2001, Ph.D. dissertation (University of Barcelona). Ibañes, M., J. García-Ojalvo, R. Toral, and J. M. Sancho, 1999, "Noise-induced phase separation: Mean-field results," Phys. Rev. E 60, 3597.

Ibañes, M., J. García-Ojalvo, R. Toral, and J. M. Sancho, 2000, "Dynamics and scaling of noise-induced domain growth," Eur. Phys. J. B 18, 663.

Ibañes, M., J. García-Ojalvo, R. Toral, and J. M. Sancho, 2001, "Noise-induced scenario for inverted phase diagrams," Phys. Rev. Lett. 87, 020601.

Imbihl, R., and G. Ertl, 1995, "Oscillatory kinetics in heterogeneous catalysis," Chem. Rev. (Washington, D.C.) 95, 697.

Inchiosa, M. E., and A. R. Bulsara, 1995, "Nonlinear dynamic elements with noisy sinusoidal forcing: Enhancing response via nonlinear coupling," Phys. Rev. E 52, 327.

Jensen, R. V., 1998, "Synchronization of randomly driven nonlinear oscillators," Phys. Rev. E 58, R6907.

Jia, X., H. M. Liao, L. Q. Zhou, and Q. Ouyang, 2004, "Properties of wave propagations induced by temporal noise in a subexcitable medium," Physica D 199, 194.

Jiang, Y., and H. Xin, 2000, "Coherent resonance in a one-way coupled system,” Phys. Rev. E 62, 1846.

John, T., R. Stannarius, and U. Behn, 1999, "On-off intermittency in stochastically driven electrohydrodynamic convection in nematics," Phys. Rev. Lett. 83, 749.

Jung, P., 1997, "Thermal waves, criticality, and selforganization in excitable media," Phys. Rev. Lett. 78, 1723.

Jung, P., U. Behn, E. Pantazelou, and F. Moss, 1992, "Collective response in globally coupled bistable systems," Phys. Rev. A 46, R1709.

Jung, P., A. Cornell-Bell, K. S. Madden, and F. Moss, 1998, "Noise-induced spiral waves in astrocyte syncytia show evidence of self-organized criticality," J. Neurophysiol. 79, 1098.

Jung, P., and P. C. Gailey, 2000, "The heartbeat of extended clocks," Ann. Phys. 9, 697.

Jung, P., and G. Mayer-Kress, 1995a, "Noise controlled spiral growth in excitable media," Chaos 5, 458.

Jung, P., and G. Mayer-Kress, 1995b, "Spatiotemporal stochastic resonance in excitable media," Phys. Rev. Lett. 74, 2130.

Jung, P., and J. W. Shuai, 2001, "Optimal sizes of ion channel clusters," Europhys. Lett. 56, 29.

Kádár, S., J. Wang, and K. Showalter, 1998, "Noise-supported traveling waves in sub-excitable media," Nature (London) 391, 770.

Kaka, S., M. R. Pufall, W. H. Rippard, T. J. Silva, S. E. Russek, and J. A. Katine, 2005, "Mutual phase-locking of microwave spin torque nano-oscillators," Nature (London) 437, 389.

Kanamaru, T., T. Horita, and Y. Okabe, 2001, "Theoretical analysis of array-enhanced stochastic resonance in the diffusively coupled FitzHugh-Nagumo equation," Phys. Rev. E 64, 031908.

Kardar, M., G. Parisi, and Y. C. Zhang, 1986, "Dynamic scaling of growing interfaces," Phys. Rev. Lett. 56, 889.

Kawai, R., X. Sailer, L. Schimansky-Geier, and C. Van den Broeck, 2004, "Macroscopic limit cycle via pure noiseinduced phase transitions," Phys. Rev. E 69, 051104.

Kim, S., S. H. Park, and H.-B. Pyo, 1999, "Stochastic resonance in coupled oscillator systems with time delay," Phys. Rev.
Lett. 82, 1620.

Kim, S., S. H. Park, and C. S. Ryu, 1998, "Colored-noiseinduced multistability in nonequilibrium phase transitions," Phys. Rev. E 58, 7994.

Kiss, I. Z., Y. Zhai, J. L. Hudson, C. Zhou, and J. Kurths, 2003, "Noise enhanced phase synchronization and coherence resonance in sets of chaotic oscillators with weak global coupling," Chaos 13, 267.

Kitahara, K., and M. Imada, 1978, "On the kinetic equations for binary mixtures," Suppl. Prog. Theor. Phys. 64, 65.

Kortluke, O., V. Kuzovkov, and W. von Niessen, 2002, "Internal spatiotemporal stochastic resonance in the presence of weak noise," Phys. Rev. E 66, 036139.

Krawiecki, A., and T. Stemler, 2003, "Stochastic resonance with spatiotemporal signal controlled by time delays," Phys. Rev. E 68, 061101.

Krawiecki, A., A. Sukiennicki, and R. A. Kosinski, 2000, "Stochastic resonance and noise-enhanced order with spatiotemporal periodic signal," Phys. Rev. E 62, 7683.

Kuhnert, L., 1986, “A new optical photochemical memory device in a light-sensitive chemical active medium," Nature (London) 319, 393.

Kuramoto, Y., 1981, "Rhythms and turbulence in populations of chemical oscillators," Physica A 106, 128.

Kurrer, C., and K. Schulten, 1995, "Noise-induced synchronous neuronal oscillations," Phys. Rev. E 51, 6213.

Kwon, O., H.-H. Jo, and H.-T. Moon, 2005, "Effect of spatially correlated noise on coherence resonance in a network of excitable cells," Phys. Rev. E 72, 066121.

Kwon, O., and H.-T. Moon, 2002, "Coherence resonance in small-world networks of excitable cells," Phys. Lett. A 298, 319.

Lai, C. H., and C. Zou, 1998, "Synchronization of chaotic maps by symmetric common noise," Europhys. Lett. 43, 376.

Lai, Y.-C., and Y.-R. Liu, 2005, "Noise promotes species diversity in nature," Phys. Rev. Lett. 94, 038102.

Lai, Y.-C., and Z. Liu, 2001, "Noise-enhanced temporal regularity in coupled chaotic oscillators," Phys. Rev. E 64, 066202. Lai, Y.-C., Z. Liu, A. Nachman, and L. Zhu, 2004, "Suppression of jamming in excitable systems by aperiodic stochastic resonance," Int. J. Bifurcation Chaos Appl. Sci. Eng. 14, 3519.

Landa, P. S., A. A. Zaikin, and L. Schimansky-Geier, 1998, "Influence of additive noise on noise-induced phase transitions in nonlinear chains," Chaos, Solitons Fractals 9, 1367.

Langer, J. S., 1971, "Theory of spinodal decomposition in alloys," Ann. Phys. (N.Y.) 65, 53.

Lee, K. J., E. C. Cox, and R. E. Goldstein, 1996, "Competing patterns of signaling activity in Dictyostelium discoideum," Phys. Rev. Lett. 76, 1174.

Lesmes, F., D. Hochberg, F. Morán, and J. Pérez-Mercader, 2003, "Noise-controlled self-replicating patterns," Phys. Rev. Lett. 91, 238301.

Limberis, L., and R. J. Stewart, 2000, "Toward kinesinpowered microdevices," Nanotechnology 11, 47.

Lindner, B., J. García-Ojalvo, A. Neiman, and L. SchimanskyGeier, 2004, "Effects of noise in excitable systems," Phys. Rep. 392, 321.

Lindner, B., and L. Schimansky-Geier, 2001, "Transmission of noise coded versus additive signals through a neuronal ensemble," Phys. Rev. Lett. 86, 2934.

Lindner, J. F., M. Bennett, and K. Wiesenfeld, 2006, "Potential energy landscape and finite-state models of array-enhanced 
stochastic resonance," Phys. Rev. E 73, 031107.

Lindner, J. F., B. J. Breen, M. E. Wills, A. R. Bulsara, and W. L. Ditto, 2001, "Monostable array-enhanced stochastic resonance," Phys. Rev. E 63, 051107.

Lindner, J. F., S. Chandramouli, A. R. Bulsara, M. Löcher, and W. L. Ditto, 1998, "Noise enhanced propagation," Phys. Rev. Lett. 81, 5048.

Lindner, J. F., B. K. Meadows, W. Ditto, M. E. Inchiossa, and A. R. Bulsara, 1996, "Scaling laws for spatiotemporal synchronization and array enhanced stochastic resonance," Phys. Rev. E 53, 2081.

Lindner, J. F., B. K. Meadows, W. L. Ditto, M. E. Inchiosa, and A. R. Bulsara, 1995, "Array enhanced stochastic resonance and spatiotemporal synchronization," Phys. Rev. Lett. 75, 3.

Liu, Z., and Y.-C. Lai, 2001, "Coherence resonance in coupled chaotic oscillators," Phys. Rev. Lett. 86, 4737.

Löcher, M., N. Chatterjee, F. Marchesoni, W. L. Ditto, and E. R. Hunt, 2000, "Noise sustained propagation: Local versus global noise," Phys. Rev. E 61, 4954.

Löcher, M., D. Cigna, and E. R. Hunt, 1998, "Noise sustained propagation of a signal in coupled bistable electronic elements," Phys. Rev. Lett. 80, 5212.

Löcher, M., G. A. Johnson, and E. R. Hunt, 1996, "Spatiotemporal stochastic resonance in a system of coupled diode resonators," Phys. Rev. Lett. 77, 4698.

Lorenzo, M. N., and V. Pérez-Muñuzuri, 1999, "Colored-noiseinduced chaotic array synchronization," Phys. Rev. E 60, 2779 .

Lorenzo, M. N., and V. Pérez-Muñuzuri, 2001, "Influence of low intensity noise on assemblies of diffusively coupled chaotic cells," Chaos 11, 371.

Lorenzo, M. N., M. A. Santos, and V. Pérez-Muñuzuri, 2003, "Spatiotemporal stochastic forcing effects in an ensemble consisting of arrays of diffusively coupled Lorenz cells," Chaos 13, 913.

Louvergneaux, E., C. Szwaj, G. Agez, P. Glorieux, and M. Taki, 2004, "Experimental evidence of absolute and convective instabilities in optics," Phys. Rev. Lett. 92, 043901.

Lythe, G. D., 1996, "Domain formation in transitions with noise and a time-dependent bifurcation parameter," Phys. Rev. E 53, R4271.

Mainen, Z. F., and T. J. Sejnowski, 1995, "Realiability of spike timing in neocortical neurons," Science 268, 1503.

Makse, H., S. Havlin, M. Schwartz, and H. E. Stanley, 1996, "Method for generating long-range correlations for large systems," Phys. Rev. E 53, 5445.

Malescio, G., 1996, "Noise and synchronization in chaotic systems," Phys. Rev. E 53, 6551.

Mangioni, S., R. Deza, H. S. Wio, and R. Toral, 1997, "Disordering effects of color in nonequilibrium phase transitions induced by multiplicative noise," Phys. Rev. Lett. 79, 2389.

Mangioni, S. E., R. Deza, R. Toral, and H. S. Wio, 2000, "Nonequilibrium phase transitions induced by multiplicative noise: Effects of self-correlation," Phys. Rev. E 61, 223.

Mangioni, S. E., and H. S. Wio, 2005, "Interplay between noise and boundary conditions in pattern formation in adsorbed substances," Phys. Rev. E 71, 056203.

Mannella, R., 2004, “Quasisymplectic integrators for stochastic differential equations,” Phys. Rev. E 69, 041107.

Manneville, P., 1990, Dissipative Structures and Weak Turbulence (Academic, London).

Marchesoni, F., L. Gammaitoni, and A. R. Bulsara, 1996, "Spatiotemporal stochastic resonance in a $\phi^{4}$ model of kink- antikink nucleation," Phys. Rev. Lett. 76, 2609.

Maritan, A., and J. R. Banavar, 1994, "Chaos, noise and synchronization,” Phys. Rev. Lett. 72, 1451.

Marro, J., and R. Dickman, 1998, Nonequilibrium Phase Transitions and Critical Phenomena (Cambridge University Press, Cambridge, UK).

Martí, A. C., J. M. Sancho, and F. Sagués, 1997, "Langevin approach to generate synthetic turbulent flows," Phys. Fluids 9, 1078.

Martin, G., 1990, "Atomic mobility in Cahn's diffusion model," Phys. Rev. B 41, 2279.

Maselko, J., and K. Showalter, 1991, "Chemical waves in inhomogeneous excitable media," Physica D 49, 21.

Meyer, C. W., G. Ahlers, and D. S. Cannell, 1987, "Initial stages of pattern formation in Rayleigh-Bénard convection," Phys. Rev. Lett. 59, 1577.

Mikhailov, A. S., 1979, "Noise-induced phase transition in a biological system with diffusion," Phys. Lett. 73A, 143.

Mikhailov, A. S., 1994, Foundations of Synergetics I: Distributed Active Systems, 2nd ed. (Springer, Berlin).

Minai, A. A., and T. Anand, 1998, "Chaos-induced synchronization in discrete-time oscillators driven by a random input," Phys. Rev. E 57, 1559.

Montejo, N., M. N. Lorenzo, V. Pérez-Villar, and V. PérezMuñuzuri, 2005, "Noise correlation length effects on a Morris-Lecar neural network," Phys. Rev. E 72, 011902.

Morillo, M., J. Gómez-Ordoñez, and J. M. Casado, 1995, "Stochastic resonance in a mean-field model of cooperative behavior," Phys. Rev. E 52, 316.

Müller, R., K. Lippert, A. Kuehnel, and U. Behn, 1997, "Firstorder nonequilibrium phase transition in a spatially extended system," Phys. Rev. E 56, 2658.

Muñoz, M. A., 2004, "Nonequilibrium phase transitions and multiplicative noise," in Advances in Condensed Matter and Statistical Mechanics, edited by E. Korutcheva and R. Cuerno (Nova Science Publishers, Hauppauge, NY), p. 34.

Muñoz, M. A., F. Colaiori, and C. Castellano, 2005, "Meanfield limit of systems with multiplicative noise," Phys. Rev. E 72, 056102.

Muñuzuri, A. P., M. Dolnik, A. M. Zhabotinsky, and I. R. Epstein, 1999, "Control of the chlorine dioxide-iodinemalonic acid oscillating reaction by illumination," J. Am. Chem. Soc. 121, 8065.

Nagumo, J., S. Arimoto, and S. Yoshizawa, 1962, "An active pulse transmission line simulating nerve axon," Proc. IRE 50, 2061.

Neda, Z., A. Rusz, E. Ravasz, P. Lakdawala, and P. M. Gade, 1999, "Spatial stochastic resonance in one-dimensional Ising systems," Phys. Rev. E 60, R3463.

Neiman, A., P. I. Saparin, and L. Stone, 1997, "Coherence resonance at noisy precursors of bifurcations in nonlinear dynamical systems," Phys. Rev. E 56, 270.

Neiman, A., and L. Schimansky-Geier, 1995, "Stochastic resonance in two coupled bistable systems," Phys. Lett. A 197, 379.

Neiman, A., L. Schimansky-Geier, A. Cornell-Bell, and F. Moss, 1999, "Noise-enhanced phase synchronization in excitable media," Phys. Rev. Lett. 83, 4896.

Neiman, A. B., and D. F. Russell, 2002, "Synchronization of noise-induced bursts in noncoupled sensory neurons," Phys. Rev. Lett. 88, 138103.

Nicolis, C., G. Nicolis, and H. L. Frisch, 1998, "Periodic forceenhanced decay of a metastable state: A case of stochastic 
resonance in a spatially extended system," Phys. Lett. A $\mathbf{2 4 9}$, 443.

Novikov, E. A., 1965, "Functionals and the random-force method in turbulence theory," Sov. Phys. JETP 20, 1290.

Ohtaki, M., T. Tanaka, and K. Miyakawa, 2004, "Noiseinduced phase locking in coupled coherence-resonance oscillators," Phys. Rev. E 70, 056219.

Ottino, J. M., 1989, The Kinematics of Mixing: Stretching, Chaos and Transport (Cambridge University Press, Cambridge, UK).

Pálsson, E., and E. C. Cox, 1996, "Origin and evolution of circular waves and spirals in Dictyostelium discoideum territories," Proc. Natl. Acad. Sci. U.S.A. 93, 1151.

Panfilov, A. V., and A. N. Rudenko, 1987, "Two regimes of scroll ring drift in the three-dimensional active media," Physica D 28, 215.

Panja, D., 2004, "Effects of fluctuations on propagating fronts," Phys. Rep. 393, 87.

Park, K., Y.-C. Lai, Z. Liu, and A. Nachman, 2004, "Aperiodic stochastic resonance and phase synchronization," Phys. Lett. A 326, 391.

Parrondo, J. M. R., C. Van den Broeck, J. Buceta, and J. de la Rubia, 1996, "Noise-induced spatial patterns," Physica A 224, 153.

Pei, X., L. Wilkens, and F. Moss, 1996, "Noise-mediated spike timing precision from aperiodic stimuli in an array of Hodgkin-Huxley-type neurons," Phys. Rev. Lett. 77, 4679.

Perazzo, R., L. Romanelli, and R. Deza, 2000, "Fault tolerance in noise-enhanced propagation," Phys. Rev. E 61, R3287.

Perc, M., 2005, "Spatial coherence resonance in excitable media," Phys. Rev. E 72, 016207.

Perez-Muñuzuri, V., R. Deza, K. Fraedrich, T. Kunz, and F. Lunkeit, 2005, "Coherence resonance in an atmospheric global circulation model," Phys. Rev. E 71, 065602.

Pérez-Muñuzuri, V., F. Sagués, and J. M. Sancho, 2000, "Lifetime enhancement of scroll rings by spatiotemporal fluctuations," Phys. Rev. E 62, 94.

Pham, J., K. Pakdaman, and J. F. Vibert, 1998, "Noise-induced coherent oscillations in randomly connected neural networks," Phys. Rev. E 58, 3610.

Pikovsky, A., M. Rosenblum, and J. Kurths, 2003, Synchronization. A Universal Concept in Nonlinear Sciences (Cambridge University Press, Cambridge, UK).

Pikovsky, A., A. Zaikin, and M. A. de la Casa, 2002, "System size resonance in coupled noisy systems and in the Ising model," Phys. Rev. Lett. 88, 050601.

Pikovsky, A. S., 1994, "Comment on 'Chaos, noise and synchronization,'” Phys. Rev. Lett. 73, 2931.

Pikovsky, A. S., and J. Kurths, 1997, "Coherence resonance in a noise-driven excitable system," Phys. Rev. Lett. 78, 775.

Postnov, D. E., S. K. Han, T. G. Yim, and O. V. Sosnovtseva, 1999, "Experimental observation of coherence resonance in cascaded excitable systems," Phys. Rev. E 59, R3791.

Postnov, D. E., O. V. Sosnovtseva, S. K. Han, and W. S. Kim, 2002, "Noise-induced multimode behavior in excitable systems," Phys. Rev. E 66, 016203.

Punckt, C., M. Bolscher, H. H. Rotermund, A. S. Mikhailov, L. Organ, N. Budiansky, J. R. Scully, and J. L. Hudson, 2004, "Sudden onset of pitting corrosion on stainless steel as a critical phenomenon," Science 305, 1133.

Qiu, X.-L., and G. Ahlers, 2005, "Dynamics of fluctuations below a stationary bifurcation to electroconvection in the planar nematic liquid crystal N4," Phys. Rev. Lett. 94, 087802.
Rabbiosi, I., A. J. Scroggie, and G.-L. Oppo, 2003, "Stochastic resonance in the presence of spatially localized structures," Phys. Rev. E 68, 036602.

Ramazza, P. L., S. Boccaletti, A. Giaquinta, E. Pampaloni, S. Soria, and F. T. Arecchi, 1996, "Optical pattern selection by a lateral wave-front shift,” Phys. Rev. A 54, 3472.

Ramírez-Piscina, L., A. Hernández-Machado, and J. M. Sancho, 1993, "Fluctuations in domain growth-GinzburgLandau equations with multiplicative noise," Phys. Rev. B 48, 119.

Rao, N. J., J. D. Borwankar, and D. Ramkrishna, 1974, "Numerical solution of Ito integral equations," SIAM J. Control 12, 124.

Rappel, W.-J., and A. Karma, 1996, "Noise-induced coherence in neural networks," Phys. Rev. Lett. 77, 3256.

Raser, J. M., and E. K. O'Shea, 2005, "Noise in gene expression: Origins, consequences, and control," Science 309, 2010. Rehberg, I., S. Rasenat, M. de la Torre Juárez, W. Schopf, F. Horner, G. Ahlers, and H. R. Brand, 1991, "Thermally induced hydrodynamic fluctuations below the onset of electroconvection," Phys. Rev. Lett. 67, 596.

Reigada, R., F. Sagués, I. M. Sokolov, J. M. Sancho, and A. Blumen, 1997, "Fluctuation-dominated kinetics under stirring," Phys. Rev. Lett. 78, 741.

Reimann, P., 2002, "Brownian motors: Noisy transport far from equilibrium," Phys. Rep. 361, 57.

Risken, H., 1984, The Fokker-Planck Equation (Springer, Berlin).

Rocco, A., J. Casademunt, U. Ebert, and W. van Saarloos, 2002, "Diffusion coefficient of propagating fronts with multiplicative noise," Phys. Rev. E 65, 012102.

Romero, A. H., and J. M. Sancho, 1999, "Generation of short and long range temporal correlated noises," J. Comput. Phys. 156, 1.

Rosenfeld, N., J. W. Young, U. Alon, P. S. Swain, and M. B. Elowitz, 2005, "Gene regulation at the single-cell level," Science 307, 1962.

Rotem, A., and E. Moses, 2006, "Magnetic stimulation of curved nerves," IEEE Trans. Biomed. Eng. 53, 414.

Rowe, A. C. H., and P. Etchegoin, 2001, "Experimental observation of stochastic resonance in a linear electronic array," Phys. Rev. E 64, 031106.

Russell, D. F., L. A. Wilkens, and F. Moss, 1999, "Use of behavioural stochastic resonance by paddle fish for feeding," Nature (London) 402, 291.

Sagués, F., F. Arias, and M. S. Miguel, 1988, "Nonlinear effects in the dynamics of transient pattern formation in nematics," Phys. Rev. A 37, 3601.

Sagués, F., and I. R. Epstein, 2003, "Nonlinear chemical kinetics," Dalton Trans. 7, 1201.

Sagués, F., M. S. Miguel, and J. M. Sancho, 1984, "Nonmarkovian dynamics of stochastic differential equations with quadratic noise," Z. Phys. B: Condens. Matter 55, 269.

Sánchez, E., M. A. Matías, and V. Pérez-Muñuzuri, 1997, "Analysis of synchronization of chaotic systems by noise: An experimental study," Phys. Rev. E 56, 4068.

Sancho, J. M., and A. Sánchez, 2000, "External fluctuations in front dynamics with inertia: The overdamped limit," Eur. Phys. J. B 16, 127.

Santagiustina, M., P. Colet, M. San Miguel, and D. Walgraef, 1997, "Noise-sustained convective structures in nonlinear optics," Phys. Rev. Lett. 79, 3633.

Santos, M. A., and J. M. Sancho, 1999, "Noise induced fronts," 
Phys. Rev. E 59, 98.

Santos, M. A., and J. M. Sancho, 2001, "Front dynamics in the presence of spatio-temporal structured noises," Phys. Rev. E 64, 016129.

Santos, M. A., J. M. Sancho, and P. W. Lamberti, 2001, "Front propagation in smectic- $\mathrm{C}^{*}$ liquid crystals in the presence of stochastic electric and magnetic fields," Eur. Phys. J. B 24, 135.

Sanz-Anchelergues, A., A. M. Zhabotinsky, I. R. Epstein, and A. P. Muñuzuri, 2001, "Turing pattern formation induced by spatially correlated noise,” Phys. Rev. E 63, 056124.

Sarmiento, A., R. Reigada, A. H. Romero, and K. Lindenberg, 1999, "Enhanced pulse propagation in nonlinear arrays of oscillators," Phys. Rev. E 60, 5317.

Satake, A., T. Kubo, and Y. Iwasa, 1998, "Noise-induced regularity of spatial wave patterns in subalpine abies forests," J. Theor. Biol. 195, 465.

Schimansky-Geier, L., and C. Zulicke, 1991, "Kink propagation induced by multiplicative noise," Z. Phys. B: Condens. Matter 82, 157.

Schmid, G., I. Goychuk, and P. Hänggi, 2001, "Stochastic resonance as a collective property of ion channel assemblies," Europhys. Lett. 56, 22.

Semwogerere, D., and M. F. Schatz, 2002, "Evolution of hexagonal patterns from controlled initial conditions in a Bénard-Marangoni convection experiment," Phys. Rev. Lett. 88, 054501.

Sendiña-Nadal, I., S. Alonso, V. Pérez-Muñuzuri, M. GómezGesteira, V. Pérez-Villar, L. Ramírez-Piscina, J. Casademunt, J. M. Sancho, and F. Sagués, 2000, "Brownian motion of spiral waves driven by spatiotemporal structured noise," Phys. Rev. Lett. 84, 2734.

Sendiña-Nadal, I., M. Gómez-Gesteira, V. Pérez-Muñuzuri, V. Pérez-Villar, J. Armero, J. C. L. Ramírez-Piscina, F. Sagués, and J. M. Sancho, 1997, "Wave competition in excitable modulated media," Phys. Rev. E 56, 6298.

Sendiña-Nadal, I., A. Muñuzuri, D. Vives, V. Pérez-Muñuzuri, J. Casademunt, L. Ramírez-Piscina, J. M. Sancho, and F. Sagués, 1998, "Wave propagation in a medium with disordered excitability," Phys. Rev. Lett. 80, 5437.

Sendiña-Nadal, I., and V. Pérez-Muñuzuri, 2001, "Noiseenhanced wave train propagation in unexcitable media," Int. J. Bifurcation Chaos Appl. Sci. Eng. 11, 2837.

Shardlow, T., 2004, "Nucleation of waces in excitable media by noise," Multiscale Model. Simul. 3, 151.

Sharpe, J. P., N. Sungar, M. Swaney, K. Carrigan, and S. Wheeler, 2003, "Stochastic resonance on two-dimensional arrays of bistable oscillators in a nonlinear optical system," Phys. Rev. E 67, 056222.

Shew, W. L., H. A. Coy, and J. F. Lindner, 1999, "Taming chaos with disorder in a pendulum array," Am. J. Phys. 67, 703.

Shuai, J. W., and P. Jung, 2002, "Optimal intracellular calcium signaling,” Phys. Rev. Lett. 88, 068102.

Sigeti, D., and W. Horsthemke, 1989, "Pseudo-regular oscillations induced by external noise,” J. Stat. Phys. 65, 1217.

Snyder, H. L., P. Meakin, and S. Reich, 1983, "Dynamical aspects of phase separation in polymer blends," Macromolecules 16, 757.

Sosnovtseva, O. V., A. I. Fomin, D. E. Postnov, and V. S. Anishchenko, 2001, "Clustering of noise-induced oscillations," Phys. Rev. E 64, 026204.

Spagnolo, B., A. Fiasconaro, and D. Valenti, 2003, "Noise induced phenomena in Lotka-Volterra systems," Fluct. Noise
Lett. 3, L177.

Stanley, H. E., 1971, Introduction to Phase Transitions and Critical Phenomena (Oxford University, New York).

Stegemann, G., A. G. Balanov, and E. Schöll, 2005, "Noiseinduced pattern formation in a semiconductor nanostructure,” Phys. Rev. E 71, 016221.

Steinbock, O., P. Kettunen, and K. Showalter, 1995, “Anisotropy and spiral organizing centers in patterned excitable media," Science 269, 1857.

Stocks, N. G., 2000, "Suprathreshold stochastic resonance in multilevel threshold systems," Phys. Rev. Lett. 84, 2310.

Stratonovich, R. L., 1967, Topics in the Theory of Random Noise (Gordon and Breach, New York), Vols. 1 and 2.

Süel, G. M., J. Garcia-Ojalvo, L. M. Liberman, and M. B. Elowitz, 2006, "An excitable gene regulatory circuit induces transient cellular differentiation," Nature (London) 440, 545. Swift, J., and P. C. Hohenberg, 1977, "Hydrodynamic fluctuations at the convective instability," Phys. Rev. A 15, 319.

Tanabe, S., T. Shimokawa, S. Sato, and K. Pakdaman, 1999, "Response of coupled noisy excitable systems to weak stimulation," Phys. Rev. E 60, 2182.

Tessone, C. J., A. Scire, R. Toral, and P. Colet, 2007, "Theory of collective firing induced by noise or diversity in excitable media," Phys. Rev. E 75, 016203.

Toral, R., 1995, "Computational field theory and pattern formation," in Lecture Notes in Physics, edited by P. L. Garrido and J. Marro (Springer, Berlin), Vol. 448, p. 3.

Toral, R., and A. Chakrabarti, 1990, "Numerical determination of the phase diagram for the $\varphi^{4}$ model in two dimensions," Phys. Rev. B 42, 2445.

Toral, R., C. Mirasso, and J. Gunton, 2003, "System size coherence resonance in coupled FitzHugh-Nagumo models," Europhys. Lett. $\mathbf{6 1}, 162$.

Toral, R., C. R. Mirasso, E. Hernández-García, and O. Piro, 2001, "Analytical and numerical studies of noise-induced synchronization of chaotic systems," Chaos 11, 665.

Tripathy, G., A. Rocco, J. Casademunt, and W. van Saarloos, 2001, "Universality class of fluctuating pulled fronts," Phys. Rev. Lett. 86, 5215.

Tsimring, L. S., and A. Pikovsky, 2001, "Noise-induced dynamics in bistable systems with delay," Phys. Rev. Lett. 87, 250602.

Turing, A. M., 1952, "The chemical basis of morphogenesis," Philos. Trans. R. Soc. London, Ser. B 327, 37.

Tyson, J. J., and P. C. Fife, 1980, "Target patterns in a realistic model of the Belousov-Zhabotinsky reaction," J. Chem. Phys. 73, 2224.

Uchida, A., R. McAllister, and R. Roy, 2004, "Consistency of nonlinear system response to complex drive signals," Phys. Rev. Lett. 93, 244102.

Ullner, E., A. Zaikin, J. García-Ojalvo, R. Báscones, and J. Kurths, 2003, "Vibrational resonance and vibrational propagation in excitable systems," Phys. Lett. A 312, 348.

Ullner, E., A. Zaikin, J. García-Ojalvo, and J. Kurths, 2003, "Noise-induced excitability in oscillatory media," Phys. Rev. Lett. 91, 180601.

Van den Broeck, C., J. M. Parrondo, and R. Toral, 1994, "Noise-induced nonequilibrium phase transitions," Phys. Rev. Lett. 73, 3395.

Van den Broeck, C., J. M. R. Parrondo, J. Armero, and A. Hernández-Machado, 1994, "Mean field model for spatially extended systems in the presence of multiplicative noise," Phys. Rev. E 49, 2639. 
Van den Broeck, C., J. M. R. Parrondo, R. Toral, and R. Kawai, 1997, "Nonequilibrium phase transitions induced by multiplicative noise," Phys. Rev. E 55, 4084.

van Kampen, N. G., 1992, Stochastic Processes in Physics and Chemistry (North-Holland, Amsterdam).

van Saarloos, W., 1989, "Front propagation into unstable states. 2. Linear versus nonlinear marginal instability and rate of convergence," Phys. Rev. A 39, 6367.

Vilar, J. M. G., and J. M. Rubí, 1997, "Spatiotemporal stochastic resonance in the Swift-Hohenberg equation," Phys. Rev. Lett. 78, 2886.

Vilar, J. M. G., and J. M. Rubí, 2000, “Ordering periodic spatial structures by non-equilibrium fluctuations," Physica A 277, 327.

Vodonos, B., R. Weill, A. Gordon, A. Bekker, V. Smulakovsky, O. Gat, and B. Fischer, 2004, "Formation and annihilation of laser light pulse quanta in a thermodynamic-like pathway," Phys. Rev. Lett. 93, 153901.

Volkov, E., M. N. Stolyarov, A. A. Zaikin, and J. Kurths, 2003, "Coherence resonance and polymodality in inhibitory coupled excitable oscillators," Phys. Rev. E 67, 066202.

Volkov, E. I., E. Ullner, A. A. Zaikin, and J. Kurths, 2003, "Frequency-dependent stochastic resonance in inhibitory coupled excitable systems," Phys. Rev. E 68, 061112.

von Haeften, B., R. Deza, and H. S. Wio, 2000, "Enhancement of stochastic resonance in distributed systems due to a selective coupling," Phys. Rev. Lett. 84, 404.

von Haeften, B., G. Izús, S. Mangioni, A. D. Sánchez, and H. S. Wio, 2004, "Stochastic resonance between dissipative structures in a bistable noise-sustained dynamics," Phys. Rev. E 69, 021107.

Walgraef, D., 1997, Spatio-temporal Pattern Formation (Springer, New York).

Wang, J., S. Kádár, and K. Showalter, 1999, "Noise-driven avalanche behavior in subexcitable media," Phys. Rev. Lett. 82, 855.

Wang, M., Z. Hou, and H. Xin, 2005, "Optimal network size for Hodgkin-Huxley neurons," Phys. Lett. A 334, 93.

Wang, Y., D. T. W. Chik, and Z. D. Wang, 2000, "Coherence resonance and noise-induced synchronization in globally coupled Hodgkin-Huxley neurons," Phys. Rev. E 61, 740.

Wehner, S., P. Hoffmann, D. Schmeisser, H. R. Brand, and J. Kuppers, 2005, "Spatiotemporal patterns of external noiseinduced transitions in a bistable reaction-diffusion system: Photoelectron emission microscopy experiments and modeling," Phys. Rev. Lett. 95, 038301.

Wiesenfeld, K., 1985, "Noisy precursors of nonlinear instabilities," J. Stat. Phys. 38, 1071.

Wiesenfeld, K., and F. Moss, 1995, "Stochastic resonance and the benefits of noise: From ice ages to crayfish and SQUIDs," Nature (London) 373, 33.

Winfree, A. T., 1973, "Scroll-shaped waves of chemical activity in three-dimensions," Science 181, 937.

Winfree, A. T., and S. H. Strogatz, 1984, "Organizing centres for three-dimensional chemical waves," Nature (London) 311, 611.

Wio, H. S., 1996, "Stochastic resonance in a spatially extended system," Phys. Rev. E 54, R3075.

Wolff, J., A. G. Papathanasiou, H. H. Rotermund, G. Ertl, M. A. Katsoulakis, X. Li, and I. G. Kevrekidis, 2003, "Wave initiation through spatiotemporally controllable perturbations,"
Phys. Rev. Lett. 90, 148301.

Wu, M., G. Ahlers, and D. S. Cannell, 1995, "Thermally induced fluctuations below the onset of Rayleigh-Bénard convection," Phys. Rev. Lett. 75, 1743.

Wu, M., and C. D. Andereck, 1990, "Effects of external noise on the Frédericksz transition in a nematic liquid crystal," Phys. Rev. Lett. 65, 591.

Zaikin, A., J. García-Ojalvo, R. Báscones, E. Ullner, and J. Kurths, 2003, "Doubly stochastic coherence via noise-induced symmetry in bistable neural models," Phys. Rev. Lett. 90, 030601.

Zaikin, A., D. Topaj, and J. García-Ojalvo, 2002, "Noiseenhanced propagation of bichromatic signals," Fluct. Noise Lett. 2, L47.

Zaikin, A. A., J. García-Ojalvo, and L. Schimansky-Geier, 1999, "Nonequilibrium first-order phase transition induced by additive noise," Phys. Rev. E 60, R6275.

Zaikin, A. A., J. García-Ojalvo, L. Schimansky-Geier, and J. Kurths, 2002, "Noise induced propagation in monostable media,” Phys. Rev. Lett. 88, 010601.

Zaikin, A. A., J. Kurths, and L. Schimansky-Geier, 2000, "Doubly stochastic resonance," Phys. Rev. Lett. 85, 227.

Zaikin, A. A., K. Murali, and J. Kurths, 2001, "Simple electronic circuit model for doubly stochastic resonance," Phys. Rev. E 63, 020103.

Zaikin, A. A., and L. Schimansky-Geier, 1998, "Spatial patterns induced by additive noise," Phys. Rev. E 58, 4355.

Zaks, M., A. Neiman, S. Feistel, and L. Shimanski-Geier, 2003, "Noise-controlled oscillations and their bifurcations in coupled phase oscillators," Phys. Rev. E 68, 066206.

Zaks, M. A., X. Sailer, L. Schimansky-Geier, and A. B. Neiman, 2005, "Noise induced complexity: From subthreshold oscillations to spiking in coupled excitable systems," Chaos 15, 026117.

Zhang, Y., G. Hu, and L. Gammaitoni, 1998, "Signal transmission in one-way coupled bistable systems: Noise effect," Phys. Rev. E 58, 2952.

Zhou, C., and J. Kurths, 2002a, "Noise-induced phase synchronization and synchronization transitions in chaotic oscillators," Phys. Rev. Lett. 88, 230602.

Zhou, C., and J. Kurths, 2002b, "Spatiotemporal coherence resonance of phase synchronization in weakly coupled oscillators," Phys. Rev. E 65, 040101.

Zhou, C., J. Kurths, and B. Hu, 2001, "Array-enhanced coherence resonance: Nontrivial effects of heterogeneity and spatial independence of noise," Phys. Rev. Lett. 87, 098101.

Zhou, C., J. Kurths, and B. Hu, 2003, "Frequency and phase locking of noise-sustained oscillations in coupled excitable systems: Array-enhanced resonances," Phys. Rev. E 67, 030101.

Zhou, C., J. Kurths, I. Z. Kiss, and J. L. Hudson, 2002, "Noiseenhanced phase synchronization of chaotic oscillators," Phys. Rev. Lett. 89, 014101.

Zhou, C., J. Kurths, Z. Neufeld, and I. Z. Kiss, 2003, "Noisesustained coherent oscillation of excitable media in a chaotic flow," Phys. Rev. Lett. 91, 150601.

Zhou, L. Q., X. Jia, and Q. Ouyang, 2002, “Experimental and numerical studies of noise-induced coherent patterns in a subexcitable medium,” Phys. Rev. Lett. 88, 138301.

Zhou, Y., and P. Jung, 2000, "Noise and complexity in threedimensional excitable media," Europhys. Lett. 49, 695. 